\title{
Clinical application of quantitative SPECT in patient specific dosimetry and beta cell quantification
}

Wietske Woliner - van der Weg 
CLINICAL APPLICATION OF

QUANTITATIVE SPECT

IN PATIENT SPECIFIC DOSIMETRY

AND BETA CELL QUANTIFICATION

\section{Proefschrift}

ter verkrijging van

de graad van doctor aan de Universiteit Twente, op gezag van de rector magnificus, prof.dr. H. Brinksma

volgens besluit van het College voor Promoties in het openbaar te verdedigen

op woensdag 27 mei 2015 om 12.45 uur

door

Wietske Woliner - van der Weg geboren op 27 maart 1987

te Leeuwarden 


\section{Contents}

\section{Supervisors:}

C.H. Slump (UT, Enschede)

M. Gotthardt (Radboudumc, Nijmegen)

\section{Co-supervisor:}

E.P. Visser (Radboudumc, Nijmegen)

ISBN

978-94-6259-684-9

Cover and Lay-out

Promotie In Zicht, Arnhem

Prin

Ipskamp Drukkers, Enschede

Copyright @ W. Woliner - van der Weg, 2015

All rights reserved. No part of this dissertation may be reproduced or transmitted in any form or by any means, electronic or mechanical, including photocopying,

recording or any information storage or retrieval system without permission from

the author, or when appropriate, from the publisher of the publications.

\section{List of abbreviations}

General introduction and outline

Chapter 1 Challenges in quantitative SPECT

Chapter 2 Predictive patient-specific dosimetry and individualized dosing of pretargeted radioimmunotherapy in patients with advanced colorectal cancer

Adapted from: EJNMMI. 2014;41(8):1593-602. Epub 2014/03/20

Chapter 3 Tumor and red bone marrow dosimetry: comparison of methods for prospective treatment planning in pre-targeted radioimmunotherapy

Adapted from: EJNMMI Physics. 2014 1:104. Epub 2015/02/24

Chapter 4 Non-invasive quantification of the beta cell mass by SPECT with ${ }^{111}$ In-exendin

Adapted from: Diabetologia. 2014;57(5):950-9. Epub 2014/02/04.

Chapter 5 Quantified pancreatic uptake of the ${ }^{111}$ In-exendin in patients with type 1 diabetes and healthy controls In preparation

Chapter 6 A 3D-printed anatomical kidney and pancreas phantom for optimizing SPECT/CT acquisition and reconstruction settings in beta cell imaging using ${ }^{111}$ In-exendin Submitted

Chapter 7 Integration of micro- and macro-dosimetry for calculation of radiation doses to the islets of Langerhans due to imaging with radiolabeled exendin In preparation

General discussion and future perspectives

Summary

Samenvatting

Dankwoord

About the author 


\section{List of abbreviations}

AC attenuation correction

absorbed dose

administered activity

beta cell mass

biological effective dose

bone marrow dose

bispecific antibody

collimator correction

colorectal cancer

computed tomography

glucagon-like peptide

$\begin{array}{ll}\text { GLP-1 } & \text { glucagon-like peptide } 1 \\ \text { GLP-1R } & \text { glucagon-like peptide } 1 \text { receptor }\end{array}$

GLP-1R glucagon-like peptide

HTP hydroxytryptophan

$\begin{array}{ll}\text { LOR } & \text { line of response } \\ \text { LV } & \text { lumbar vertebrae }\end{array}$

MAP maximum a posteriori

MIRD medical internal radiation dose (committee)

MRI magnetic resonance imaging

MRP median root prior

NEMA-IQ national electrical manufacturers association - image quality (phantom)

OLINDA software package based on S-values

OSEM ordered subset expectation maximization

PET positron emission computed tomography

PRIT pre-targeted radioimmunotherapy

RBM red bone marrow

RIT radioimmunotherapy

ROI region of interest

SC scatter correction

SPECT single photon emission computed tomography

TEW triple energy window

T1D, T2D type 1 diabetes, type 2 diabetes

VOI volume of interest

2D, 3D two-dimensional, three-dimensional

3D-RD three-dimensional radiobiological dosimetry (software)

$\% \mathrm{ID} / \mathrm{g}$ percentage of the injected dose per gram of tissue 
General introduction,

outline and research questions 


\section{Use of radionuclides in nuclear medicine: visualization and treatment}

Radioactivity is widely utilized for both diagnosis and treatment of patients It enables visualization of biological and physiological processes and selective treatment by local delivery of high radiation doses.

Two types of cameras are the workhorses for visualization of radioactivity distribution:

1. The gamma camera produces two-dimensional (2D), also called planar, images of the distribution of photon-emitting (photons are gamma-radiation) radionuclides and when used in rotating mode it provides three-dimensional (3D) imaging called single photon emission computed tomography (SPECT).

2. The positron emission tomograph (PET scanner) creates 3D images of the spatial distribution of positron-emitting radionuclides. In this case, two photon originating from the annihilation of the positron with an electron are detected simultaneously.

These techniques are further described in chapter 1.

Both PET and SPECT hold the promise for quantification of the amount of radioactivity in a certain volume of tissue (1). Quantification of this amount of activity, also called the 'uptake', can be valuable for both diagnosis and treatment, for example to quantify the uptake as an estimate for the amount of a certain type of cells in an organ (e.g. beta cells in the pancreas, as in chapter 4 and 5). Another relatively advanced use of quantitative images, is to use them for the calculation of radiation absorbed doses. This is called image-based dosimetry and can be applied to optimize an intended amount of activity, high enough for treatment, by predicting the targeting of the compound, and to calculate the radiation dose to surrounding healthy tissue (see chapter 2 and 3). This illustrates the value of quantitative analysis of SPECT images for clarification of processes in individua patients and enabling patient tailored treatment.

Nevertheless, for correct acquisition and interpretation of quantitative images, three different factors should be taken into account: the physics of the image formation, the choice of the imaging protocol parameters and biological and physiological factors (2). The influence of physics in the image acquisition can be summarized as the effect of attenuation of radiation before it reaches the camer and the effect of scattering of radiation within the body, in collimators and in detectors. These factors and others are further discussed in chapter 1 
In both PET and SPECT, physics plays a role in quantification, but PET-imaging wa optimized for quantification already decades ago. From the moment PET was used in the clinic, it has resulted in reconstructed data in units of radioactivity per unit volume $\left(\mathrm{kBq} / \mathrm{cm}^{3}\right)(1,3)$, while SPECT was long considered as 'non-quantitative' (3). Recent developments in SPECT image reconstruction resulted in uncomplicated use of iterative reconstruction techniques and methods for adequate scatter an attenuation correction. This made quantitative use of SPECT images possible within errors of less than 10\% (1). The challenges of quantification for SPECT are described in chapter 1

\section{The value of quantitative use of SPECT images}

The developments enabling quantitative SPECT are valuable since they facilitate broader use of quantification in nuclear medicine by also allowing single gammaemitting radionuclides next to positron-emitters. In some cases, using a gammaemitting radionuclide instead of a positron-emitter is advantageous or even necessary; the selection of the radionuclide, and therefore the imaging modality, is based on both technical (e.g. labeling efficiency, preferred imaging modality, aim of the study), practical (e.g. availability, costs) and safety concerns (e.g. radiation dose to the patient).

In general SPECT cameras are more widely available than PET cameras and SPECT is more affordable than PET (3). Also, physical half-lives for many SPECT radionuclides are longer and therefore more compatible with the biological halflives of the physiologic process of interest (3). These are reasons to prefer SPECT in particular applications.

\section{Clinical application of quantification, and outline of this thesis, and research questions}

Understanding the technical challenges in quantitative use of SPECT images is of major importance for correct interpretation of the images and requires awareness of influences of technical, protocol, biological or physiological origin. Therefore chapter 1 handles the question 'What challenges are to be considered for quantitative use of SPECT images and dosimetry?'. The reader is introduced into the differences between SPECT and PET imaging, challenges of quantitative use of the images, the rationale of treatment with radionuclides and different quantification and dosimetry methods.
This is followed by examples of (pre-)clinical application of quantitative use of SPECT images and dosimetry, in chapters 2 to 7 .

Dosimetry can be used to calculate which amount of activity should be administered to reach a dose to the target tissue that is high enough for therapeutic efficacy and that that can be administered safely without exposing organs at risk to excessive doses. In chapter 2 and 3 data of a clinical, so-called, pre-targeted radioimmunotherapy (PRIT) study in patients with colorectal cancer, and the dosimetry used for this study is described. In the PRIT-study, patients are treated with the gamma- and beta-emitter ${ }^{17} \mathrm{Lu}$, after pre-therapeutic administration of ${ }^{111} \mathrm{In}$, which was coupled to the same peptide as ${ }^{177} \mathrm{Lu}$ and therefore enabled predictive dosimetry. The pre-targeting strategy is used to improve tumor-to-background ratios, compared to using directly radiolabeled antibodies which require several days to localize tumors effectively. Pre-targeting techniques achieve rapid accretion of the radionuclide in the tumor in combination with rapid blood clearance by first administering a non-radiolabeled bispecific antibody.

In chapter 2, blood-based dosimetry and planar ${ }^{111}$ In-images, made with a gamma-camera, are used for estimation of the radiation dose to the bone marrow and the kidneys, in order to avoid toxicity due to ${ }^{177} \mathrm{Lu}$ administration. The results of this prospective dosimetry (estimation of the ${ }^{177} \mathrm{Lu}$ dose, based on the pre-therapy ${ }^{11}$ In data) is compared to the results of retrospective blood-based and plana image-based ${ }^{177} \mathrm{Lu}$ dosimetry. This chapter discusses the value of predictive dosimetry based on image- and blood-based quantification methods, and answers the question 'What is the predictive value of planar image-based and blood-based bone marrow dosimetry for patient tailored PRIT planning?'

An alternative method for blood- or planar image-based bone marrow dosimetry is described in chapter 3 , where we used the 3D ${ }^{177} \mathrm{Lu}$ SPECT image from the PRIT-study for retrospective Monte Carlo based dosimetry, to get insight in unexpected clinical findings. Some of the patients showed signs of bone marrow toxicity, while the predicted bone marrow dose (based on blood-based and 2D-image based dosimetry) was lower than the expected toxic dose. This Mont Carlo based dosimetry method is more complex and time-consuming, therefore the question raised: 'What is the additional value of using Monte Carlo based bone marrow dosimetry based on SPECT images, compared to blood-based dosimetry and planar image-based dosimetry, in relation to bone marrow toxicity?

In chapter 4, 5, 6 and 7 quantification of the amount of pancreatic beta cells is the major topic. Beta cells are the insulin producing cells in the pancreas, and play a key role in diabetes. While beta cell function can be measured easily (e.g by monitoring blood glucose levels), addition of a method for non-invasive in vivo quantification of the beta cell mass (BCM) can largely contribute to the knowledge 
about the pathophysiology and treatment of diabetes by enabling investigation of the relation between BCM and function. Therefore radiolabeled exendin, which specifically targets the glucacon-like-1 (GLP-1) receptor, expressed on beta cells, was developed. Exendin labeled with ${ }^{111}$ In can be visualized in vivo with SPECT. The main question in the work described in chapter 4 is ' $\mathrm{Can}{ }^{111}$ In-exendin be used for beta cell quantification?'. To answer this, preclinical work for the validation of ${ }^{111}$ In-exendin for beta cell quantification and the first clinical findings with ${ }^{111}$ In-exendin are presented.

Chapter 5 describes the first complete clinical study about beta cell quantification with ${ }^{111}$ In-exendin. In this study, SPECT images were used to investigate the beta cells in 10 patients with type 1 diabetes (T1D) and 10 healthy volunteers. With these first clinical experience the question 'Do T1D patients have a lower pancreatic uptake of exendin than healthy volunteers and is the uptake range in line with the expected range in $B C M$ ? Would the use of radiolabeled exendin for beta cell characterization be feasible on a larger scale?' can be answered.

Since exendin has a relatively high uptake in the kidneys compared to the pancreas, quantification of the pancreatic uptake is even more complicated than SPECT quantification in general. Therefore a method was needed to mimick the patient situation, for thorough investigation of the effect of different settings in reconstruction software and optimization of the image reconstruction protocol. The question raised: 'Can a 3D-printed phantom be used for optimizing acquisition and reconstruction settings for quantitative SPECT in beta cell quantification?'. Therefore, in chapter $\mathbf{6}$ we describe how a 3D printed phantom for beta cell quantification was developed, and how this was used for optimizing the acquisition and reconstruction settings in beta cell imaging with radiolabeled exendin.

The final work in chapter $\mathbf{7}$ was performed to calculate the dose to islets of Langerhans (including the beta cells) in patients imaged with radiolabeled exendin. Since no suitable dosimetry model was available, the question raised "Can we develop an islet dosimetry model that can be adjusted to different biological situations, for estimation of islet doses after administration of radiolabeled exendin? To develop this model different sources were used: preclinical data (chapter 4), clinical data (from study in chapter 5) and different dosimetry methods (see chapter 1) were combined. After development, the model was applied to different situations to answer the question: 'What is the radiation dose to the islets of Langerhan after administration of radiolabeled exendin?
In the general discussion and future perspectives, answers to the questions raised above will be discussed, and ideas for future developments will be launched.

\section{References}

Ritt P, Vija H, Hornegger J, Kuwert T. Absolute quantification in SPECT. European journal of nuclear medicine and molecular imaging. 2011:38 Suppl 1:S69-77. Epub 2011/04/13.

Frey EC, Humm JL, Ljungberg M. Accuracy and precision of radioactivity quantification in nuclear medicine images. Seminars in nuclear medicine. 2012;42(3):208-18. Epub 2012/04/06.

WP. An evidencebased review of quantitative SPECT imaging and potential clinical applications. Journal of nuclear. 2013;54(1):83-9. Epub 2013/01/04. 
1

Challenges in quantitative SPECT 


\section{Abstract}

Although both positron emission tomography (PET) and single photon emission computed tomography (SPECT) hold the promise for quantification, SPECT quantification is more complicated. SPECT imaging requires the use of a collimator and the detectors rotate around the patient, therefore detection efficiency is lower. Also adequate correction for attenuation is more complicated than in PET. The energy windows selected for acquisition, and the collimator used depend on the radionuclide. All these factors contribute to the reliability of quantification of SPECT images.

The reconstruction method used can highly contribute to the quality of the quantification by providing corrections for scatter, attenuation and collimator modeling.

Besides the acquisition and reconstruction protocol, patient specific factors (e.g. movement) and practical issues (e.g. using software, which is not compatible with scaling factors in DICOM files of other vendors) can influence quantification. For reliable interpretation of the quantification results, also biological influences should be understood: what is the biological parameter which is quantified with quantification of the uptake of the radionuclide, and how can this be influenced? When taking these factors into consideration, absolute or relative quantification can be performed, and radiation doses can be calculated.

Examples of the use of quantification are described in this thesis: the use of dosimetry for treatment planning, investigation of dose-response relations, quantification of the beta cell mass and optimizing acquisition and reconstruction protocols.

\section{Introduction}

For dosimetry (calculation of the radiation dose) in nuclear medicine, quantification of the radiotracer distribution is essential. Also, quantification can be useful for diagnostics, for example for quantification of the pancreatic beta cells, as in chapter 4 and 5 .

Ex vivo investigation of the tracer distribution is less complicated than in vivo quantification. In preclinical studies, the uptake in different organs is often measured ex vivo. After dissection, uptake in organs can be quantified in a gamma counter, and with autoradiography of tissue slices a more detailed distribution can be visualized. In autoradiography a phosphor imaging plate is exposed to the radiation from tissue slices and read out of this plate visualizes the trace distribution in the tissue (this technique is used in chapter 4 and 7). In vivo quantification in patients relies on imaging: single photon emission computed tomography (SPECT) or positron emission tomography (PET)

Although both PET and SPECT hold the promise for quantification (1), quantification of SPECT images is more challenging. Even with these additional challenges, quantification of SPECT images is attractive, because it enables quantification of gamma-emitting radionuclides. For PET imaging the use of a positron emitting radionuclide is required, but for technical, practical or safety reasons the use of a gamma-emitter can be preferred, or even required.

Differences between the challenges for SPECT and PET quantification are largely influenced by the differences in the image acquisition technique. To elucidate the challenges for SPECT, first both image acquisition methods will be discussed.

Then the challenges will be described. These include a broad spectrum, since the final results of quantitative evaluation of the radiotracer distribution can be influenced by all parts of the quantification workflow, including preparation imaging, reconstruction and analysis

To conclude, also different applications of quantification will be noted, including dosimetry

\section{Image acquisition}

\section{Gamma camera/ SPECT}

Gamma-emitting radionuclides that emit photons (gamma-radiation) within the energy range of 80 to $500 \mathrm{keV}$, for example ${ }^{99 \mathrm{~m}} \mathrm{Tc},{ }^{111} \mathrm{In},{ }^{131} \mathrm{I}$ and ${ }^{177} \mathrm{Lu}$, can be visualized with a gamma camera (2). The energy has to be sufficiently high for 
penetrating the patient's body without too much attenuation and low enough for enabling detection in the camera. Figure 1 shows the process of SPECT imaging.

Before absorption in a sodium iodine (NaI) scintillation crystal of the camera, the photons first pass the collimator (see figure 2). This collimator is a grid avoiding detection of non-perpendicularly entering photons, and therefore enabling to assign detected photons to a certain line of response (LOR). Without a collimator photons originating from a broad area within the patient can be absorbed anywhere in the scintillation crystal, resulting in unusable, completely blurred images. A disadvantage, however, is reduction of the efficiency, since only a small fraction of the gamma radiation that hits the collimator surface passes through (1). Ideally, only perpendicularly entering photons are detected (figure 2 , examples A and C). In practice, some photons will be scattered in the collimator (figure 2 , example B), or penetrate the septum (figure 2, example D). Therefore differen collimators are in use and selection of the optimal collimator is based on the energy of the photons to be detected. Photo-mulitiplier tubes behind the crysta amplify the signal and finally an analog-to-digital (ADC) converter digitizes the signal and assigns the X-Y location (2) (see figure 2).

Since the pulses produced have a finite duration and the system has certain processing duration, the next pulse cannot be processed as a separate event if the first is not yet finished. The period in which no next detection can be processed is

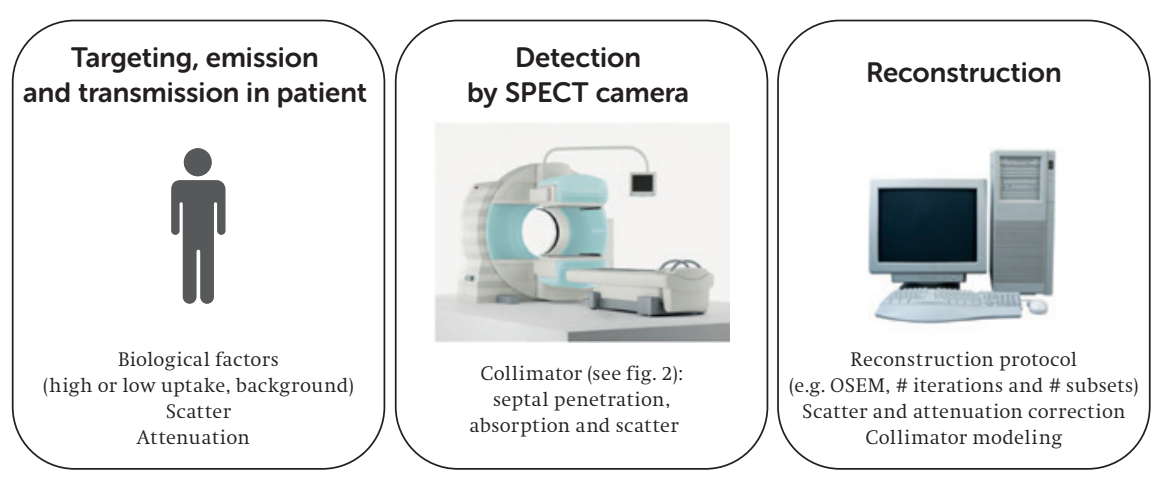

Figure 1 Single photon emission computed tomography: after injection of the gammaemitting radiotracer, the radionuclide emits photons in the patient. These photons are detected by the SPECT camera. The acquisition data has to be reconstructed to retrieve 3-dimensional images of the distribution of the radionuclide in the patient. OSEM is ordered subset expectation maximization, a reconstruction method.

source SPECT-camera image. http://www.siemens.com/about/sustainability/en/environmental-portfolio/ products-solutions/healthcare/symbia-t6-and-t16.htm

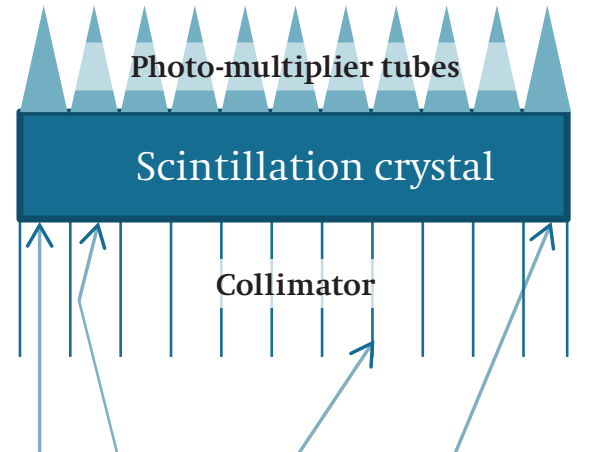

A B

D

Figure 2 Schematic overview of a single photon emission computed tomograph (SPECT) detector. The grid of the collimator enables detection of perpendicular entering photons (A) non-perpendicular entering photons should be absorbed by septa of the collimator (C) Septal scattering (B) or septal penetration (D) leads incorrect detection of photons. Afte detection in the scintillation chrystal, the signal is amplified by the photo-multiplier tubes.

called dead time. Because of this limitation, photons can remain undetected and counts get lost when the activity is too high. This can lead to an underestimation of the activity in the resulting image $(2,3)$. Dead time should therefore be considered and eventually be corrected for.

In the image acquisition protocol, energy windows are defined (see figure 3). Each separately defined window results in one image, representing the signal detected within this window. The intensity in the pixels is the time-integrated signal within the window for that location. Depending on the number of photopeaks required for adequate image formation, and the method for attenuation correction, multiple photopeak and scatter windows (for corrections) can be defined (see paragraph 'Reconstructions' for the implications of window choices). Although often only one photopeak window is needed, for certain radionuclides a second photopeak window can be used for increasing image quality and improving quantification (e.g. for ${ }^{111}$ In, see figure 2). Scatter windows are used to correct for scatter by measuring the numbers of photons detected just above and below the photopeak window. The average counts in the adjacent scatter windows are subtracted from the photopeak window, as it is assumed that this is the number of counts within the photopeak window that does not origin from the photopeak. (see also paragraph 'Scatter') In contrast to PET, in SPECT different energy windows need to be defined for different radionuclides. Selection of these energy windows 


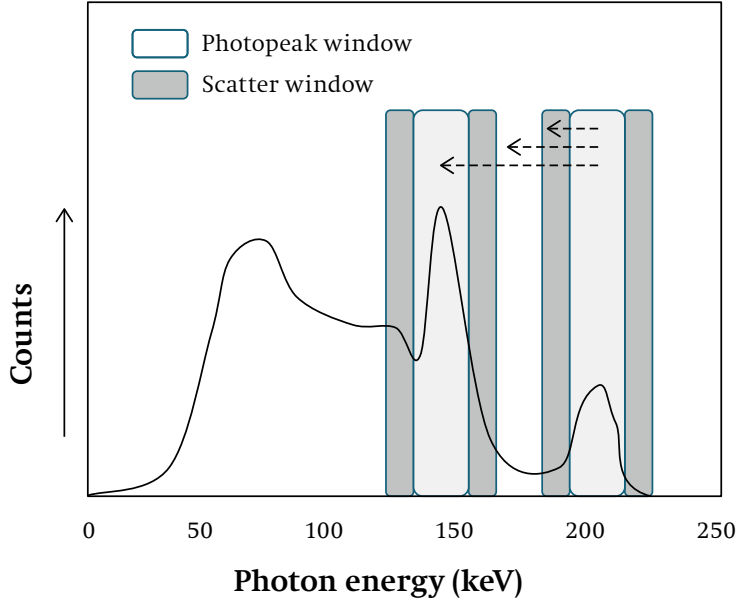

Figure 3 Schematic overview of an ${ }^{11}$ In spectrum, representing the photons detected by a gamma-camera. The two photopeak windows include the peaks induced by the photons emitted by ${ }^{111} \mathrm{I}$. The dashed arrows indicate examples of how an ${ }^{11}$ In photon that lost energy due to scattering can end outside the (original) photopeak window: it can end up in a scatter window, outside the defined energy windows, or in a lower photopeak window.

influence image quality, and whether scatter can be corrected with data resulting from scatter windows or not.

Performing SPECT, the gamma camera detectors rotate around the patient and from each angle, data are acquired as planer images (projections). The detectors can rotate in 'step-and-shoot' mode, in which the camera only acquires data when halted at specified angles. The alternative is to rotate in continuous motion. This reduces the study time, because there is no delay in acquisition due to movement. In theory this continuous motion leads to a more blurred image, in practice this effect is limited.

Another movement variation is perpendicular to the direction of rotation: the camera can move with a fixed radius of rotation of the camera heads, or follow the body contour. Following the body contour improves spatial resolution, and slightly improves the detection efficiency. Since this detection efficiency depends on the distance between the camera and the object, using the 'body contour' setting for quantitative images requires adequate correction for each angle.

In PET imaging the detector the detector is circular, with a fixed diameter Therefore, in contrast to SPECT, in PET no choices have to be made for the detector position and movement.
In the last decade, integrated SPECT/ computed tomography (CT) cameras became available. These are not only useful for anatomical reference, but also improve quantification (see paragraphs 'attenuation' and 'scatter'). Most departments now have an integrated SPECT/CT, but addition of CT imaging increases costs and radiation dose to the patient (e.g. $1.9 \mathrm{mSv}$ for a typical low-dose CT in chapter 6), therefore the CT is only used when required for diagnosis or quantification.

In chapter 2 and 3, images acquired on a SPECT-alone camera were used, the studies in chapter 4, 5 and 6 were performed on a SPECT/CT camera.

\section{PET}

Positron-emission, for example by ${ }^{18} \mathrm{~F},{ }^{68} \mathrm{Ga}$ and ${ }^{89} \mathrm{Zr}$, leads to annihilation of the emitted positron $\left(\mathrm{e}^{+}\right)$with an electron (e) in the proximity of the emission. This process leads to formation of two $0.511 \mathrm{MeV}$ photons that move in opposite directions. These photons are absorbed by scintillators in the PET detector ring, almost simultaneously. This coincidence is used to determine the LOR, and therefore no collimator is required. The PET camera, not having to use a collimator and with a circular detector, has a much higher detection efficiency compared to SPECT. Also, attenuation correction is less complicated in PET than in SPECT, because of the coincidence detection: the origin of the emission is not needed for correction in PET, only the average attenuation in the LOR.

Nowadays most PET systems have an integrated CT. This CT is used as anatomical reference and for attenuation correction. It is common to use integrated PET/CT for all PET-investigations.

PET is not used for quantification purposes in this thesis, but in chapter 3 PET is used in the way it is most often applied in the clinic: to visualize pathophysiolog ical processes in which the glucose metabolism is increased. In chapter 3, these are colorectal tumors and metastases. Therefore the radioactive glucose analogue ${ }^{18}$ F-fluorodeoxyglucose (FDG) was administered and 1 hour after administration the uptake visualized with PET.

\section{Challenges in quantification}

Even before radiation reaches the camera, interaction with tissue leads to absorption and scatter, influencing quantification. Together with factors related to the object (patient), the acquisition technique and data processing, these can be considered as "the challenges" in quantitative use of SPECT images, and the challenges that were faced in the work described in this thesis. 


\section{Scatter}

Interaction of photons with tissue can lead to 'Compton scattering': Collision between the photon and an outer-shell electron from the tissue leads to ejection of the electron and deflection of the photon. Besides the change in direction due to this process, the photon also looses energy, and both these changes influence quantification

When the total loss of energy is so large that the photon energy is lower than the lower limit of the energy window of the photopeak, it will not be detected in the photopeak window. The photon will, correctly, be 'lost' for quantification. See figure 2 .

If the energy loss is limited, or when a photon from a higher photopeak ends up in the lower photopeak window, it will still be in a photopeak window and therefore be included in the reconstruction. In these cases, the assumed LOR does not correspond to the origin of the photon and therefore the emission will incorrectly be assigned to a different position. Because photons originating from the higher photopeak, can end up in a lower photopeak window, scatter effects are larger when multiple photopeak windows are used (4).

There are different methods for scatter correction. The TEW (triple energy window) method, in which the counts of two scatter windows (see figure 2) surrounding the photopeak window are subtracted from the photopeak, is the least complicated method, and can be accurate for quantification (5). With this method, the number counts in the photopeak window is lowered to the level of counts if there would not have been scattered photons or background activity detected. An alternative when SPECT/CT is available is, to use CT-based scatter correction, implemented in the reconstruction algorithm. In these, Monte Carlo based estimations of scatter and absorption effects are used with the patient's density map (CT). During the iterative reconstruction, the estimated effects are directly taken into account. In chapter 6 both methods are applied, TEW in Siemens' Flash 3D software and CT based scatter correction in Hermes' Hybrid Recon.

\section{Attenuation}

Attenuation is the loss of photons for detection because passing material leads to absorption and scatter of photons. Depending on the energy of the gamma-radiation, and density of the material to be passed, more or fewer photons will be absorbed by the tissue, which reduces the number of interactions with the camera. For photons with a higher energy, attenuation is lower, and in high density tissue (e.g. bone) the attenuation is higher than in low density tissue (e.g. lung). For larger objects, without correction attenuation is the single largest factor degrading the quantification accuracy in SPECT images (4).
Different methods are used for attenuation correction. The Chang-method (6) assumes a homogeneous tissue density and corrects voxel intensities with constant attenuation factor (e.g. $0.13 \mathrm{~cm}^{-1}$ for ${ }^{177} \mathrm{Lu}$, in chapter 3 ) after reconstruction. This correction is based on the body contour. The shape of the body, and thus the shape of the volume for which the correction is performed, can for example be based on the detected radiation. This method requires a relatively high enough amount of radioactivity in the background tissue, to be able to detect the body contour.

A more sophisticated method is to use a CT scan as density map, preferably acquired with an integrated camera to ensure the same patient position. With this, inhomogeneous tissue densities are included in the corrections. This method is especially preferred in areas like in the pelvis and the chest, in which tissue density is not homogeneous.

Note that acquisition of the CT takes only seconds, while the SPECT acquisition needs at least minutes, and in some studies even more than half an hour. Therefore movement, like breathing, can influence the correction. Especially in the chest and the upper part of the abdomen, very close to the lungs, this should be considered.

A Chang-like method, integrated in the iterative reconstruction and correcting each projection, is used for attenuation correction in chapter 2 and 3, CT based corrections were used for the other studies in this thesis.

\section{Other}

A main difference between PET and SPECT, is that SPECT imaging depends on the detection of single photons and requires the use of a collimator. The resolution of clinical SPECT cameras is generally not as good as PET and detection efficiency is lower. Especially in low-count regions this complicates quantification. In beta cell imaging (chapter 4, 5 and 6), biological factors (i.e. relatively low uptake in the pancreas and secretion of the ${ }^{111}$ In via, but also accumulation in, the kidneys) put high demands on image reconstruction and quantification. Relatively low uptake in the region of interest can be compensated by changing the image protocol: increasing amounts of radioactivity administered to the patient (at cost of a higher radiation dose) or increase of the image acquisition time (with the risk of patien motion and discomfort). In the beta cell imaging study, imaging time was increased to 45 minutes per SPECT. Quantification of the low count pancreas region, located close to the high intense kidneys remains challenging. For optimizing this quantification, phantom experiments (as in chapter 6) can be performed.

Less prominent than the technical and biological issues, but as important, is to ensure that all software and data 'speak the same language', and that all settings are correct. This seems easier than it actually is. The standard format for handling SPECT and CT data is DICOM (Digital Imaging and COmmunications in Medicine). 
For PET the use of DICOM tags like 'rescale slope' and 'offset' is defined, for SPECT this is not. Different vendors use their own tags, which may include scaling factors. The scaling is required since pixel data are saved as integers, while with the use of reals no scaling would be needed. The use of scaling factors in other DICOM-tags than used for PET was for example observed in ReSPECT (Scivis, Germany) reconstructions, as made for the work in chapters 3 and 4 . If this would not have been revealed, quantification would have been unreliable because ReSPECT used different scaling factors for the various reconstructed images. Therefore, especially when acquisition, reconstruction and/ or analysis are performed with software of different vendors, preservation of the quantitative quality should be investigated and confirmed. Also, trivial appearing options as 'preserve total projection counts' in Hermes Hybrid Recon and 'preserve negative values' in Siemens' Flash3D, can have a devastating effect on the final quantification, while these can be very useful for non-quantitative clinical applications.

For absolute quantification, standard operating procedures (SOPs) should be strictly respected, for example because the amount of activity administered to the patient should be known. Therefore either the syringe needs to be flushed with saline at the end of the administration, or the remaining activity in the syringe needs to be measured. Eventual spilling of activity should at least be mentioned, for taking this effect into account during the analysis.

Also biological processes should be considered. For example in beta cell quantification (chapter 4 and 5), the quantification depends on the uptake of radiolabeled exendin due to the working of the glucagon-like-peptide-1 (GLP-1) receptor. Changes in receptor expression can therefore influence the uptake, and lead to incorrect interpretation of quantification results.

Quality assurance and calibration of the gamma cameras is outside the scope of this work, but at least it should be noticed that for quantification, camera performance should be stable and reliable.

Finally, partial volume effect and patient movement (e.g. breathing), should, especially for quantification of smaller areas and areas in or close to the lungs, be taken into account.

This all means that for quantitative use of images, every step, from preparation up to analysis, requires additional attention.

\section{Key for enabling quantification: reconstruction protocol}

One of the keys to overcome the challenges in quantitative use of SPECT images, is the use of an advanced image reconstruction algorithm within a protocol that is able to correct for different influences. Therefore, the development of such algorithms was of vital importance for enabling quantitative use of SPECT images (1).
A relatively fast iterative reconstruction algorithm, which is suitable for quantification (4) and implemented in reconstruction software of different vendor is ordered subset expectation maximization (OSEM). The reconstruction protocol should at least enable attenuation correction. The addition of so-called collimator correction, which includes the geometric (depth-dependent) response of the collimator, is also beneficial in reducing the partial volume effect by improving image resolution $(1,7)$. Different variations on OSEM reconstructions and their influence on beta cell quantification are discussed in chapter 6 .

\section{Quantification and dosimetry}

\section{Absolute versus relative quantification}

In absolute quantification the actual amount of radioactivity in a certain volume is calculated. This is not always required; relative quantification can be sufficient for answering a clinical question.

In relative quantification, the intensity in one area is compared to another area within the patient, within other patients, or within a phantom. This still requires adequate correction for scatter and attenuation, but is independent of the calculation of a calibration factor. Relative quantification is currently the primary quantification method in general nuclear medicine (3).

In absolute SPECT quantification the calibration factor is needed to translate intensity within a certain volume in the image to the amount of radioactivity within that volume.

It is important to choose the way of quantification that is required for enabling the clinical diagnostic or therapeutic decision making. A high accuracy is always desirable, but for many applications a constant bias or deviation from the actual value is acceptable (4), and then the less complicated relative quantification might be preferred.

When absolute quantification is performed correctly, including corrections for attenuation, scatter and collimator response, an accuracy of better than $5 \%$ can be reached for ${ }^{111}$ In (4). This shows that, when all challenges that have to be overcome are considered, accurate quantification of SPECT images is indeed possible.

\section{Dosimetry}

Dosimetry is the calculation of a radiation dose, which is the amount of absorbed energy within tissue with a certain mass due to the interaction of the radiation with tissue, expressed in Gray $((\mathrm{Gy})=(\mathrm{J} / \mathrm{kg}))$. Dosimetry not only requires to know the amount of radioactivity at a certain moment; to calculate the absorbed energy, ideally the energy deposition within the volume of interest at each moment in 
time is measured. The deposition depends on the likelihood of interaction, which on its own depends on the tissue, and type and energy of the radiation.

As a more practical approach, than to measure the energy deposition at each moment, the dose calculation is based on different moments in time at which the activity in the VOI is known. Before, between and after these, an estimation is made. The deposition of energy can be estimated in different ways.

The most reliable, yet most time consuming, method is to do Monte Carlo (MC) simulations in which tissue density, type and energy of radiation are included. When many (e.g. more than 1 million) of these simulations are performed for a certain volume, this gives a reproducible estimation of the energy deposition (e.g. $<1 \%$ difference with the results of another set of MC simulations). For each image, the dose rate (the energy deposition rate within a for a certain volume of tissue $(\mathrm{Gy} / \mathrm{s}))$ is calculated. Interpolation and extrapolation for the period in which the energy is deposited leads to the dose. An example in which MC simulations are used for calculation of the absorbed dose, is the 3D-radiobiological dosimetry (3D-RD) software package (8), developed at the Johns Hopkins Medical Institute (Baltimore, MD, USA), which is used in chapter 3.

A more commonly used, less advanced, but practical method is the use of dose conversion factors, the so-called S-values, as implemented in OLINDA/EXM (9). Therefore first, for each source volume (which can be an organ, whole body or tumor), the residence time of the source $\left(\tau_{\text {source }}\right.$ in $\left.\mathrm{MBq} \times \mathrm{h} / \mathrm{MBq}=\mathrm{h}\right)$ has to be calculated by integrating the area under the time activity curve, in which the activity is represented by a fraction of the administered activity ( $\mathrm{AA}$, in $\mathrm{MBq}$ ).

The contribution of a certain source organ to a certain target organ is calculated by multiplying the AA, and the residence time of the source, with the specific S-value $\left(\mathrm{S}_{\text {target source }}\right)$. The sum of the contributions of all source organs is the absorbed dose in the target organ $\left(\mathrm{AD}_{\text {target }}\right)$ in $\mathrm{Gy}$.

$$
A D_{\text {target }}=\sum_{\text {sources }} A A \times \tau_{\text {source }} \times S_{\text {target, source }}
$$

Figure 4 gives an example of the use of S-value based dosimetry, in the way it is implemented in the islet dosimetry model in chapter 7. The kidneys contribute to the dose in the pancreas, and the pancreas itself contributes to the pancreas dose. In the model no contribution of the remainder of the body to the pancreas dose is calculated, because activity in the remainder was neglectibly low.

The S-value is influenced by the mass of the target organ, the total energy associated with the specific radiation type, and the absorbed fraction. The S-value represents the fraction of energy absorbed in the target organ compared to energy absorbed elsewhere. The sum of all S-values of one target is 1 . Note that source and

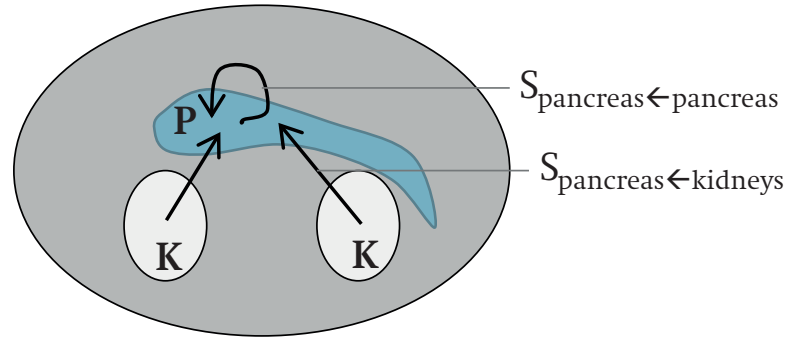

Figure 4 A schematic overview of the use of S-values for dosimetry. The kidneys (indicated with a K) contribute to the radiation dose in the pancreas (P). The value of $S$ describes the level of contribution to the dose. $S$ describes the level of contributio of the activity in the pancreas to the self-dose in the pancreas.

target organ can be the same (e.g. the dose to the liver, due to the radioactivity in the liver). S-values are based on MC simulations in anthropomorphic phantoms.

Residence times can be based on 2D or 3D images, or even on a combination of both (10). For estimation of the residence time in the blood (as a surrogate for the bone marrow), also counts within serial blood samples can be used. A more extensive description about the method is given by Stabin and Siegel (11). In chapter 2, 3 and 7 dosimetry based on S-values plays an important role.

Another method for image-based dosimetry is to use dose volume kernels (e.g. used in STRATOS, the dosimetry package of Philips' Imalytics). This method is not further discussed, because it is not used within this thesis.

\section{Use of quantification in radionuclide therapy and beta cell imaging} Quantification plays a role in both diagnostics and in therapy with radionuclides. In diagnostics it serves for quantification of an amount of cells (e.g. the beta cells, see chapter 4 and 5) or a process (e.g. the energy consumption in FDG-PET imaging). In treatment it can be used to plan or evaluate a treatment, or even to assess whether treatment with another radionuclide would be beneficial (see chapter 2 and 3).

The role of quantification in the different examples passing in this thesis is briefly described here.

\section{Prospective and retrospective dosimetry}

Before treatment with a damage-inducing radionuclide (beta or alpha-emitter), a gamma-emitting surrogate can be used to estimate the targeting of the therapeutic compound. With this, the amount of radioactivity to be administered can be chosen high enough to ensure the intended therapeutic effect, and low enough to 
limit toxicity in other, healthy, tissue. Calculation of the radiation dose before therapy, as described in chapter 2 , is called predictive dosimetry.

When a gamma-emitting radionuclide (e.g. ${ }^{177} \mathrm{Lu}$, which is a beta- and a gammaemitter) is used for therapy, also the therapy itself can be evaluated by calculating radiation doses based on several images. This is called retrospective dosimetry. In chapter 3 , retrospective dosimetry was used to study the dose-toxicity relation.

In chapter 7 model-based dosimetry is performed, which can be considered as prospective dosimetry, and can be used for estimation of the radiation dose to patient without exposing the patient to radiation.

\section{(Pre-targeted) radioimmunotherpy planning}

In order to deliver the ionizing radiation to a certain target tissue, or to visualiz this target, radionuclides can be attached to peptides that act as a vehicle with a specific target. This concept is used in radioimmunotherapy (12). To reduce the radiation dose to non-target tissue, a two-step administration (pre-targeted radioimmunotherapy, PRIT) can be advantageous. In PRIT, first an antibody with a high affinity for the tumor associated antigen is administered. When this antigen is cleared from the blood and has accumulated in the tumor tissue, a smaller radiolabeled molecule is administered as the second step. Because the compound with the radionuclide is smaller than in traditional single-step radioimmunotherapy, the clearance from the blood is faster, and therefore the dose to non-target organs will be reduced. Also for PRIT the therapy planning can be based on a gamma-emitting surrogate of the therapeutic compound. This approach is used in the clinical study in chapter 2 and 3.

\section{Quantification of radiotracer distribution, as surrogate for the beta cell distribution}

Beta cells are the insulin producing cells in the islets of Langerhans in the pancreas. A non-invasive method for quantification of amount of beta cell, also indicated as 'beta cell mass', in addition to measures of the beta cell function (insulin production), can contribute to the knowledge about changes in the beta cells during the development and treatment of diabetes. However, the islets cannot be visualized directly by magnetic resonance imaging (MRI) or CT because of their small size $(50-400 \mu \mathrm{m})$. As an alternative, the beta cells can be visualized by SPECT after administration of ${ }^{111}$ In-exendin, a highly specific radiotracer so that the signal detected is also specific for beta cells. The ${ }^{111}$ In gets trapped in the beta cells after internalization. Quantification of this uptake of ${ }^{111}$ In enables non-invasive quantification of the actual beta cell mass in vivo.

In beta cell quantification, one of the challenges is that the amount of cells to be quantified is small and in patients with diabetes, the beta cell mass is even ower than in healthy subjects. Also, relatively high activity in the kidneys complicates quantification; the tail of the pancreas is located close to the left kidney, which therefore projects over the pancreas. By measuring the effects of the use of different acquisition and reconstruction protocols with the use of a phantom, patient studies can be optimized. These challenges are faced in chapter 4, 5 and 6

\section{Conclusion}

In conclusion, the challenges of quantitative analysis of SPECT images originate rom preparation, image acquisition, reconstruction and processing. The development of OSEM reconstruction algorithms, and adequate correction for scatter, attenuation and the collimator detector response largely contribute to the potential of using SPECT for quantification. This enables use of SPECT for quantification and dosimetry in a wide variety of therapeutic and diagnostic applications, some of which will be described in the remaining chapters of this thesis.

\section{References}

Ritt P, Vija H, Hornegger J, Kuwert T. Absolute quantification in SPECT. European journal of nuclear medicine and molecular imaging. 2011;38 Suppl 1:S69-77. Epub 2011/04/13.

2. Cherry SR, Sorensen JA, Phelps ME. Physics in Nuclear Medicine. Philadelphia, PA, USA: Saunders, Elsever inc.; 2012

3. Beauregard JM, Hofman MS, Pereira JM, Eu P, Hicks RJ. Quantitative (177)Lu SPECT (QSPECT) imagin using a commercially available SPECT/CT system. Cancer imaging : the official publication of the International Cancer Imaging Society. 2011;11:56-66. Epub 2011/06/21

4. Frey EC, Humm JL, Ljungberg M. Accuracy and precision of radioactivity quantification in nuclear medicine images. Seminars in nuclear medicine. 2012;42(3):208-18. Epub 2012/04/06.

5. Bailey DL, Willowson KP. An evidence-based review of quantitative SPECT imaging and potentia clinical applications. Journal of nuclear medicine. 2013;54(1):83-9. Epub 2013/01/04.

6. Chang L-T. A Method for Attenuation Correction in Radionuclide Computed Tomography. Nuclear Science, IEEE Transactions on. 1978;25(1):638-43.

7. Kangasmaa T, Sohlberg A, Kuikka JT. Reduction of collimator correction artefacts with bayesian reconstruction in spect. International journal of molecular imaging 2011:2011:630813. Epub 2011/04/15. Sgouros G, Frey E. Wahl R, He B. Prideaux A Hobbs R. Three dimensional imagingased radiobiobeical dosimetry. Seminars in nuclear medicine. 2008;38(5):321-34. Epub 2008/07/30.

9. Stabin MG, Sparks RB, Crowe E. OLINDA/EXM: the second-generation personal computer software for internat dose assesment in nuclear medicine. Journal of nuclear medicine. 2005:46(6):1023-7. Ep

Wierts R, de Pont CD, Brans B, Mottaghy FM, Kemerink GJ. Dosimetry in molecular nuclear therapy. Methods. 2011;55(3):196-202. Epub 2011/10/01.

11. Stabin MG, Siegel JA. Physical models and dose factors for use in internal dose assessment. Health Phys. 2003;85(3):294-310. Epub 2003/08/27.

12. Gabriel M. Radionuclide therapy beyond radioiodine. Wiener medizinische Wochenschrift. 2012:162(19-20):430-9. Epub 2012/07/21. 


\section{2}

Predictive patient-specific dosimetry and individualized dosing of pretargeted radioimmunotherapy in patients with advanced colorectal cancer

Rafke Schoffelen, Wietske Woliner - van der Weg, Eric P. Visser, David M. Goldenberg, Robert M. Sharkey, William J. McBride, Chien-Hsing Chang, Edmund A. Rossi, Winette T.A. van der Graaf, Wim J.G. Oyen, and Otto C. Boerman 


\section{Abstract}

Pretargeted radioimmunotherapy (PRIT) with bispecific antibodies (bsMAb) and a radiolabeled peptide reduces the radiation dose to normal tissues. Here, we report the accuracy of an ${ }^{111}$ In-labeled pre-therapy test dose for personalized dosing of ${ }^{177}$ Lu-labeled IMP288 following pretargeting with the anti-CEA $\mathrm{x}$ anti-hapten bsMAb, TF2, in patients with metastatic colorectal cancer (CRC).

Methods: In twenty patients bone marrow absorbed doses (BMD) and doses to kidneys were predicted based on blood samples and scintigrams acquired after ${ }^{111}$ In-IMP288 injection for individualized dosing of PRIT with ${ }^{177} \mathrm{Lu}-\mathrm{IMP} 288$ Different dose schedules were studied, varying the interval between the bsMAb and peptide administration ( 5 days vs 1 day), increasing the bsMAb dose (75 mg vs $150 \mathrm{mg}$ ), and lowering the peptide dose (100 $\mathrm{\mu g}$ vs $25 \mu \mathrm{g}$ ).

Results: TF2 and ${ }^{111} \mathrm{In} /{ }^{177} \mathrm{Lu}-\mathrm{IMP} 288$ clearance was highly variable. A strong correlation was observed between peptide residence times and individual TF2 blood concentrations at the time of peptide injection (Spearman's $\rho=0.94, P<0.0001$ ). PRIT with 7.4 GBq ${ }^{177} \mathrm{Lu}-\mathrm{IMP} 288$ resulted in low radiation doses to normal tissues (BMD $<0.5$ Gy, kidney dose $<3$ Gy). Predicted ${ }^{177}$ Lu-IMP288 BMD were in good agreement with the actual measured doses (mean difference - $0.0026 \mathrm{mGy} / \mathrm{MBq}$, SD $0.028 \mathrm{mGy} / \mathrm{MBq}$ ). Hematologic toxicity was mild in most patients, with only two (10\%) having grade 3-4 thrombocytopenia. A correlation was found between platelet toxicity and BMD (Spearman's $\rho=0.58, P=0.008$ ). No non-hematologic toxicity was observed.

Conclusion: These results show that individual high activity doses in pretargeted radioimmunotherapy in patients with CEA-expressing CRC could be safely administered by predicting the radiation dose to red marrow and kidneys, based on dosimetric analysis of a test dose of TF2 and ${ }^{111}$ In-IMP288.

ClinicalTrials.gov Identifier NCT00860860

\section{Introduction}

Selective targeting of tumor-associated antigens with radiolabeled antibodies can be used for diagnosis and therapy of cancer. By combining imaging with treatment, concept designated as theranostics, targeted radionuclide therapies can be personalized. For example, diagnostic information obtained from pre-therapeutic PET or SPECT can be used to predict potential toxicity, and possibly even the efficacy of the treatment. In this way, patients can be selected for radioimmunotherapy by estimating the probability of tumor control versus the risk of toxicity of a planned treatment. This analysis also may aid in identifying the most appropriate radionuclide or cocktail of radionuclides ${ }^{90} \mathrm{Y},{ }^{177} \mathrm{Lu},{ }^{213} \mathrm{Bi}$, etc.) to delive the therapeutic dose that ensures the patient receives a unique dose optimized for their radionuclide therapy. Finally, imaging data could potentially assess the herapeutic response. The feasibility of this approach was demonstrated in patients with neuroendocrine tumors using ${ }^{111} \mathrm{In}$ - or ${ }^{68} \mathrm{Ga}$-labeled somastatin analogues for diagnosis and the same peptides labeled with ${ }^{177} \mathrm{Lu}$ - or ${ }^{90} \mathrm{Y}$ for radionuclide therapy $(1,2)$.

We recently undertook a first-in-man clinical investigation of a bispecific antibody (bsMAb) pretargeting procedure in patients with advanced colorectal cancer using a ${ }^{177} \mathrm{Lu}$-labeled hapten-peptide as the therapeutic (3). In this clinica study, the potential of dosimetric analysis of the test dose, using the same hapten-peptide radiolabeled with ${ }^{111}$ In to estimate a therapeutic dose that could be administered safely with ${ }^{177} \mathrm{Lu}$ was evaluated. Pretargeting is a strategy that was developed to improve the imaging and therapeutic characteristics of targeted radionuclides as compared to directly radiolabeled monoclonal antibodies. Radiolabeled antibodies require several days to localize tumors effectively, due to the slow pharmacokinetics and accretion of intact antibodies in tumors. These properties cause delayed visualization of tumors and increases bone marrow toxicity for therapy. Pretargeting techniques achieve rapid accretion of the radionuclide in the tumor in combination with rapid blood clearance by first administering a non-radiolabeled bsMAb. After the bsMAb has localized in the tumor and has cleared from the circulation, a small radiolabeled hapten-peptide is administered, which extravasates quickly, where it is trapped in the tumor by the bsMAb, while the remainder is cleared rapidly from the blood and is eliminated via the kidneys. The retention of the radiolabeled hapten-peptide in the tumor is increased when the peptide carries two haptens (4). In our pretargeting system, peptides are substituted with the hapten histamine-succinyl-glycine (HSG), creating a highly versatile pretargeting system, because the peptide can be conjugated with various chelating moieties (DTPA, NOTA, DOTA, $\mathrm{N}_{3}$ S-chelates, etc.), enabling the use of radionuclides, such as ${ }^{111} \mathrm{In}$ and ${ }^{99 \mathrm{~m}} \mathrm{Tc}$ for SPECT imaging $(5),{ }^{18} \mathrm{~F}$ and ${ }^{68} \mathrm{Ga}$ for PET imaging (6-9), or ${ }^{131} \mathrm{I},{ }^{90} \mathrm{Y}$, and ${ }^{177} \mathrm{Lu}$ for pretargeted radioimmunotherapy (PRIT) $(10,11)$. 
Until recently, the bsMAbs used in pretargeting were either produced by chemical conjugation of Fab-fragments or by the quadroma technology. A novel method, known as Dock-and-Lock (DNL ${ }^{\mathrm{TM}}$ ), was developed to produce humanized trivalent Fab bsMAb constructs with two binding Fab's for the tumor-associated antigen and one for the hapten on the radiolabeled peptide $(12,13)$. DNL constructs were used effectively in a pretargeting therapy setting using ${ }^{177} \mathrm{Lu} /{ }^{90} \mathrm{Y}$-labeled di-HSG-peptide in xenograft models $(10,14,15)$. We also examined a humanized anti-CEA $x$ anti-HSG DNL-constructed bsMAb,TF2, and a HSG-substituted haptenpeptide (IMP288) in a nude mouse model of peritoneal presentation of human cancer $(12,16)$. These studies showed the enhanced sensitivity and specificity of this pretargeting system compared to FDG-PET $(9,17,18)$. Furthermore, we reported that PRIT with TF2 and ${ }^{177} \mathrm{Lu}$-peptide is an effective treatment modality for colon cancer, with limited toxicity (10). Pretargeted immuno-SPECT images acquired after diagnostic ${ }^{111}$ In-IMP288 or therapeutic ${ }^{177} \mathrm{Lu}-\mathrm{IMP} 288$ administrations were compared quantitatively (15) and used to predict and monitor the therapeutic effect of PRIT. Therefore, a pre-therapy diagnostic study with the same compound could be used to estimate absorbed doses for individualized therapeutic dose assignment $(19,20)$

In this report, we examine the feasibility of an ${ }^{111}$ In-labeled pre-therapy test dose for personalized dosing and prediction of radiation dose of PRIT with TF2 and the ${ }^{177} \mathrm{Lu}-\mathrm{IMP} 288$ in a first-in-man study in metastatic colorectal cancer (CRC) patients.

\section{Methods}

\section{Patients}

The inclusion criteria were age $\geq 18$ years; histologically or serologically confirmed CEA-expressing advanced colorectal malignancies refractory to conventional treatment or without any standard therapeutic option; Eastern Cooperative Oncology Group performance status $\leq 1$; no chemotherapy, external beam radiation, immunotherapy or angiogenesis inhibitors within four weeks of study entry; adequate hematopoietic function (absolute neutrophil count $\geq 1.5 \times 10^{9} / \mathrm{L}$; platelets $\geq 150 \times 10^{9} / \mathrm{L}$ without transfusion during the previous month; hemoglobin $\geq 5.6 \mathrm{mmol} / \mathrm{L}$; adequate hepatic function (total bilirubin $\leq 2 \mathrm{x}$ upper limit of normal (ULN), aspartate transaminase (AST)/alanine transaminase (ALT) $\leq 3 \mathrm{x}$ ULN); and adequate renal function (serum creatinine $\leq 2 \times$ ULN, Cockcroft clearance $>50 \mathrm{ml} / \mathrm{min}$ ). Exclusion criteria were a life expectancy $\leq 6$ months, known brain metastases, cardiac disease with New York Heart Association classification of III or IV, or any other illness significantly affecting the patients' clinical condition. The protocol
ClinicalTrials.gov Identifier NCT00860860) was approved by the Institutiona Review Board of the Radboud University Medical Center. Written confirmed consent was obtained from all patients prior to any study-related procedures.

\section{Preparation and administration of investigational drugs}

The pretargeting agents were provided by Immunomedics (Morris Plains, NJ, USA) The clinical grade trivalent anti-CEACAM5 $\mathrm{x}$ anti-HSG bsMAb construct, TF2, and the IMP288 peptide were described previously $(8,13)$. IMP288 was labeled with ${ }^{111}$ In (Covidien, Petten, The Netherlands) at a specific activity of $185 \mathrm{MBq} / 100$ or $25 \mu \mathrm{g}$ (2.6 or $10.6 \mathrm{MBq} / \mathrm{nmol}$ ) and with ${ }^{177} \mathrm{Lu}$ (IDB Holland BV, Baarle Nassau, The Netherlands, and Isotope Technologies Garching GmbH, Garching, Germany) at a specific activity of 3.7-7.4 GBq/100 or $25 \mu \mathrm{g}$ (53-423 MBq/nmol). Radiochemica purity of the radiolabeled IMP288 preparations always exceeded 95\% using instant thin-layer chromatography and reversed phase high-performance liquid chromatography as described previously (10)

The TF2 dose (75 or $150 \mathrm{mg}$ ) was diluted in $60 \mathrm{~mL} \mathrm{0.9 \%} \mathrm{w/v} \mathrm{NaCl,} \mathrm{and}$ administered by intravenous infusion over a period of two hours. Patients received a prophylactic dose of clemastine $(2 \mathrm{mg}$ ) and $10 \mathrm{mg}$ dexamethason intravenously 15 minutes prior to start of their second TF2 infusion to suppress infusion-related symptoms. ${ }^{111} \mathrm{In}$-IMP28 8 was diluted in $10 \mathrm{~mL} 0.9 \% \mathrm{NaCl}$ and ${ }^{177} \mathrm{Lu}-\mathrm{IMP} 288$ in $20 \mathrm{~mL}$ $0.9 \% \mathrm{NaCl}$, and administered by an intravenous bolus .

\section{Study design}

Before initiating therapy, patients underwent a pre-therapy study with TF2 and ${ }^{111}$ In-labeled IMP288. The ${ }^{111}$ In-IMP288 data were used to simulate absorbed doses of ${ }^{177} \mathrm{Lu}-\mathrm{IMP} 288$ and to calculate a safe individual ${ }^{177} \mathrm{Lu}$-activity dose (see paragraph: Dosimetric calculations and individual ${ }^{177} \mathrm{Lu}$ doses). Subsequently, the therapy cycle was administered, using the same interval and bsMAb and IMP288 doses (Fig. 1A). Fou different pretargeting conditions were examined in cohorts of five patients each (Fig. 1B), which included varying the interval between the bsMAb and haptenpeptide administrations (cohort 1: 5 days $v s$ all other cohorts: 1 day), the TF2 dose (cohort 3: $150 \mathrm{mg}$ TF2 vs other cohorts: $75 \mathrm{mg}$ ), and two IMP288 doses were studied (cohort 4: $25 \mu \mathrm{g}$ IMP288 vs other cohorts: $100 \mu \mathrm{g}$ ). $25 \mu \mathrm{g}$ IMP288 was the minimal peptide dose required to label the peptide with the maximum ${ }^{177} \mathrm{Lu}$ dose, $7.4 \mathrm{GBq}$ 


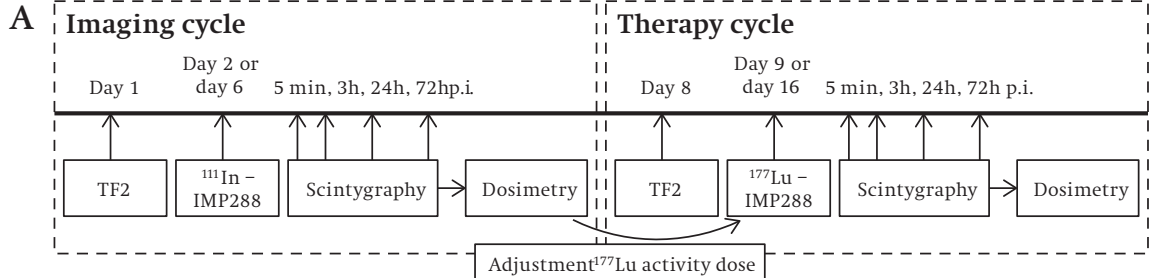

\begin{tabular}{|c|c|c|c|}
\hline Cohort & $\begin{array}{c}\text { TF2 } \\
\text { dose }\end{array}$ & Interval & IMP288 \\
\hline$(\mathrm{n}=5)$ & (mg) & (days) & Dose $(\mu g)$ \\
\hline 1 & 75 & 5 & 100 \\
\hline 2 & 75 & 1 & 100 \\
\hline 3 & 150 & 1 & 100 \\
\hline 4 & 75 & 1 & 25 \\
\hline
\end{tabular}

Figure 1 Study design. A: Patients received an imaging cycle with TF2 and ${ }^{111}$ In-IMP288 to determine the pharmacokinetics and radiation doses to the red marrow and the kidneys. Based on the dosimetry calculations, the maximum amount of ${ }^{177} \mathrm{Lu}-$ activity to be given in 4 successive doses was estimated. B: Four cohorts receiving different pretargeting conditions were studied.

\section{Pharmacokinetics}

Serum samples were collected at the end of the TF2 infusion, $30 \mathrm{~min}, 1 \mathrm{~h}, 2 \mathrm{~h}, 4 \mathrm{~h}$, $6 \mathrm{~h}$, and $24 \mathrm{~h}$ after infusion, the last sample being taken shortly before peptide infusion. TF2 concentrations were determined with a sandwich enzyme-linked immunoabsorbent assay (ELISA) developed by Immunomedics, using HSG-conjugated peptide as a capture and anti-MN-14 anti-idiotype antibody as the probe antibody as described previously (21). Blood samples were collected 2 min after ${ }^{111}$ In- and ${ }^{177} \mathrm{Lu}-\mathrm{IMP} 288$ injection, and then at $30 \mathrm{~min}, 1,2,4,24$ and $72 \mathrm{~h}$ p.i. They were counted in a gamma counter (Wizard, Pharmacia-LKB, Sweden) using appropriate energy windows, with standards prepared from the injected products. The percentage of the injected dose per gram tissue (\% ID/g) and blood residence time were calculated.

\section{Scintigraphy}

Whole-body planar scintigraphic images were acquired first, followed by SPECT imaging of the kidney region with $\geq 1$ tumor lesion in the field of view, using Siemens dual-head gamma camera (Ecam, Hoffmann Estates, IL), equipped with medium-energy collimators. Symmetric 15\% windows were used over both the 172
$\mathrm{KeV}$ and $246 \mathrm{KeV}$ energy peaks of ${ }^{111}$ In-scintigraphy, and over both 113 and $208 \mathrm{KeV}$ for ${ }^{177} \mathrm{Lu}$-scintigraphy. Scans were acquired within $15 \mathrm{~min}$ after the injection of IMP288 before voiding, $3 \mathrm{~h}$ after injection after voiding and $24 \mathrm{~h}$ and $72 \mathrm{~h}$ after injection. If scintigraphic images showed ${ }^{111}$ In-IMP288 accumulation in metastatic lesions, patients were eligible for ${ }^{177} \mathrm{Lu}$-IMP288 therapy.

\section{Dosimetric analysis and individual ${ }^{177} \mathrm{Lu}$ doses}

The ${ }^{111}$ In-IMP288 data were used as surrogates to calculate predicted radiation doses of ${ }^{17}$ Lu-IMP288, assuming identical pharmacokinetics and biodistribution of ${ }^{111}$ In-IMP288 and ${ }^{177} \mathrm{Lu}-\mathrm{IMP} 288$. Simulation of the ${ }^{111} \mathrm{In}$ scans and blood data for ${ }^{177} \mathrm{Lu}$ and calculation of residence times were performed as described previously (20). In OLINDA, the dynamic bladder model was used (bi-exponential model, bladder voiding at 3-hour interval). The dose to the red bone marrow was calculated using two methods: (I) quantification of the radioactivity in a region of interest (ROI) ove the cranium in the scintigraphic images (i.e., imaging-based method), as described previously (22); and (II) based on the radioactivity concentrations in the blood as described by Shen et al. (i.e., blood-based method) (23). For the imaging method, the residence time was divided by the fraction of the red marrow mass in the cranium to the mass in the total body, for which the default value 0.119 was taken from ICRP23's Reference Man. For the blood method, a red marrow-to-blood activity concentration of 1 was applied, as was determined for the ${ }^{177} \mathrm{Lu}-\mathrm{IMP} 288$ (24).

Since the red marrow and the kidneys were considered as the organs primarily at risk for radiation-induced toxicity, the simulated absorbed doses to these organ were used to calculate a safe total activity dose of ${ }^{177} \mathrm{Lu}$ that would deliver no more than $1.25 \mathrm{~Gy}$ to the red marrow or no more than 15 Gy to the kidneys, because these thresholds are generally accepted to be below the absorbed dose for radiation-induced toxicity $(25,26)$. For the red bone marrow, the radiation dose calculated with either the imaging- or blood-based method was used, whicheve was the highest. The total calculated ${ }^{177} \mathrm{Lu}$ activity that was assumed to be safe was divided into four equal amounts that were intended to be given on 4 separate occasions every 8 or up to 12 weeks, based on toxicity from each successive cycle. Additionally, in the first cohort, the maximum total ${ }^{177} \mathrm{Lu}$ activity dose per cycle was limited to $3.7 \mathrm{GBq}$, even when dosimetric calculations would allow a highe ${ }^{177} \mathrm{Lu}$ dose. In the next cohorts, the maximum dose per cycle was allowed to be up to $7.4 \mathrm{GBq}$. In all patients, only one treatment cycle was given, because of progression of disease. 


\section{Patient monitoring}

Patients were monitored closely during and up to eight weeks after therapy, with physical examination, clinical biochemistry, hematology, and toxicity assessment according to the Common Terminology Criteria for Adverse Events (CTCAE v3.0) Significant toxicities were defined as: grade 4 thrombocytopenia lasting for $\geq$ weeks or $\geq$ grade 3 thrombocytopenia with bleeding, grade 4 neutropenia lasting for $\geq 7$ days,or $\geq$ grade 3 neutropenia with fever at least $38.5^{\circ} \mathrm{C}$, or any $\geq$ grade 3 non-hematologic toxicity, with the exception of nausea, vomiting, and diarrhea.

\section{Statistics}

Statistical analysis was performed with GraphPad Prism software version 5.00 for Windows (GraphPad Software, San Diego USA), using two-tailed Mann Whitney test with Bonferroni correction when multiple groups were compared, Spearman's correlation tests for not normally distributed and cateoorical data, and Bland Altman plots for agreement tests. The level of significance was set at $P<0.05$.

\section{Results}

The baseline characteristics for the patients that were treated with ${ }^{177} \mathrm{Lu}-\mathrm{IMP} 288$ are summarized in Table 1. Additional safety and clinical data have been reported elsewhere (3).

\section{Pharmacokinetics}

The first cohort of patients utilized a 5-day interval, because preclinical studies in rabbits suggested this might be a suitable interval. However, TF2 cleared rapidly from the blood in all patients, with $>99 \%$ of the administered dose cleared within $24 \mathrm{~h}$. Thus, cohorts 2-4 utilized a 1-day interval. As expected, the shorter interval resulted in higher concentrations of the bsMAb at the time of peptide injection (cohort 1: 5 days vs cohort 2: 1 day, $P=0.01$, Man-Whitney) (Fig. 2A). Surprisingly, patients who received a higher TF2 dose (cohort 3: $150 \mathrm{mg}$ ) had a larger variation in the amount of bsMAb in the blood compared to those that received the lower dose (cohort 2 and 4: $75 \mathrm{mg}$ ) at $24 \mathrm{~h}$ p.i (range 0.077-1.44 $\mu \mathrm{g} / \mathrm{mL}$ vs $0.16-0.59 \mu \mathrm{g} / \mathrm{mL}$ in cohort 3 vs cohort 2 and 4, respectively). No correlation was found between TF2 blood clearance rate and CEA plasma levels. The higher variability in blood concentrations of TF2 in cohort 3 led to an expected similar high variability in blood residence time for IMP288 (cohort 3: 1.5-7.5 h vs cohort 2: 2.0-5.5 h); however, there was also considerable variability in IMP288 blood residence times for patients in cohort 4 who received the lower IMP28 8 dose with $75 \mathrm{mg}$ of TF2 (e.g., cohort 4 1.7-9.3 h) (Fig. 2B). When compared within the cohorts that received the same

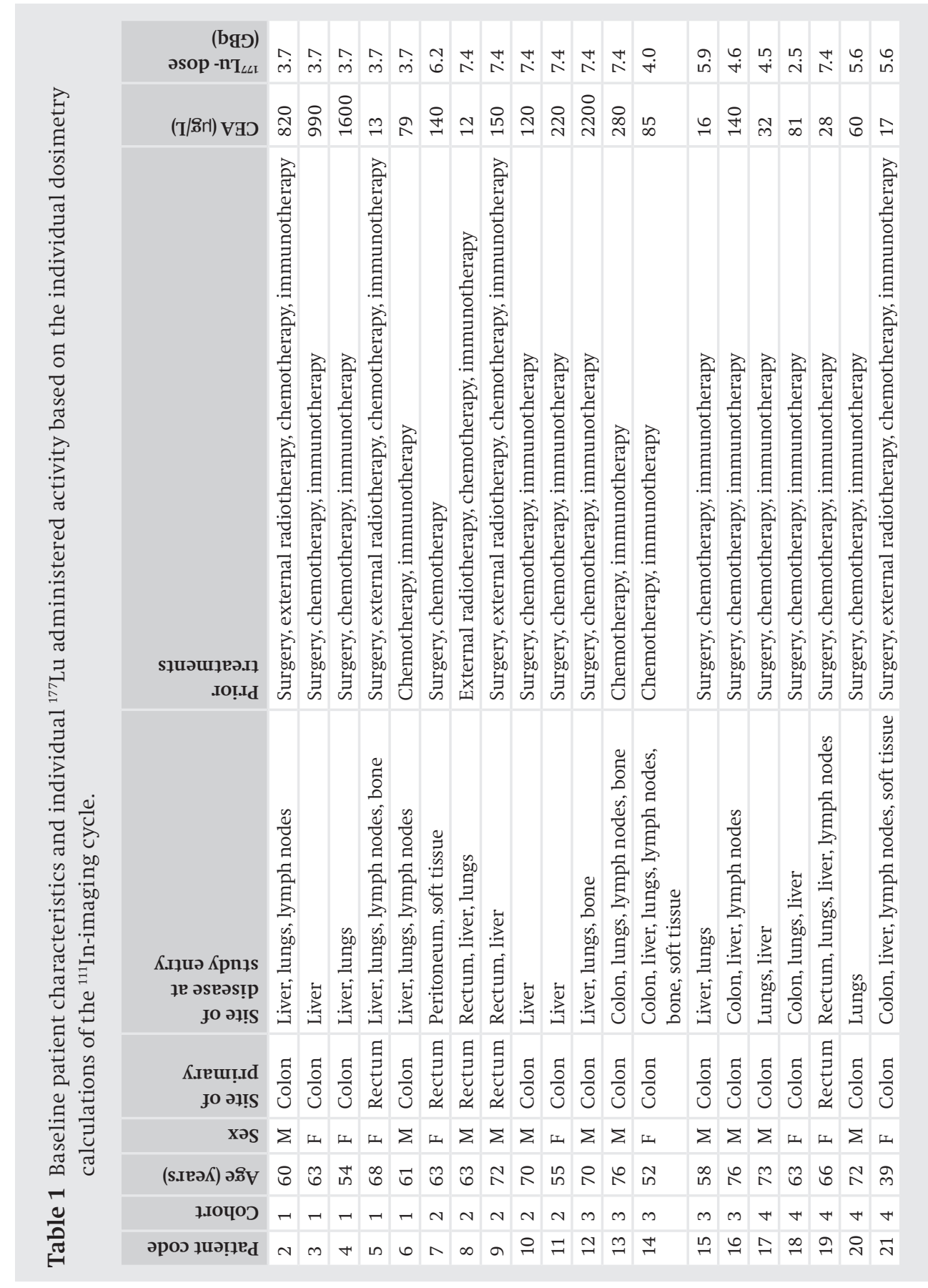


A

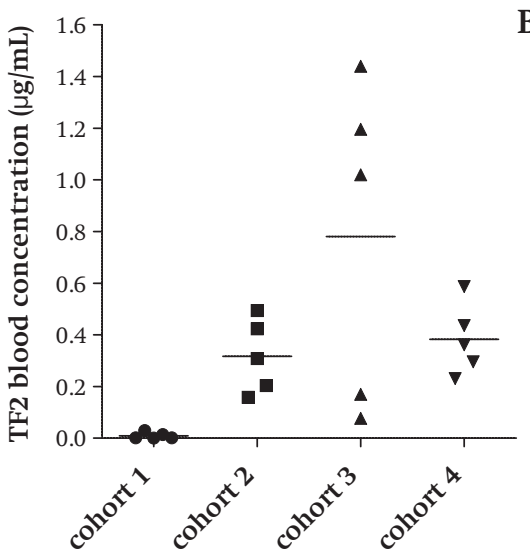

B

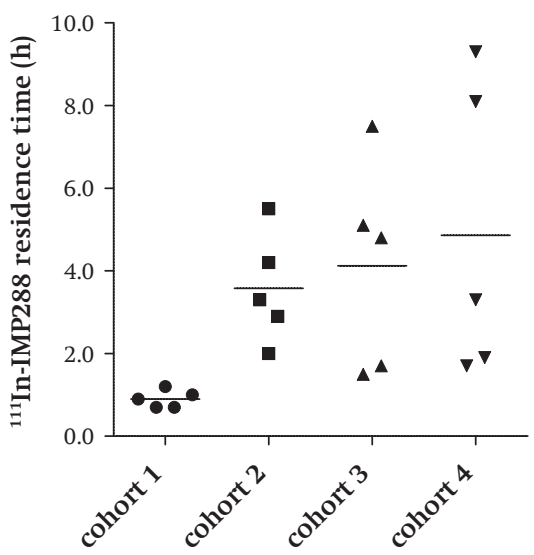

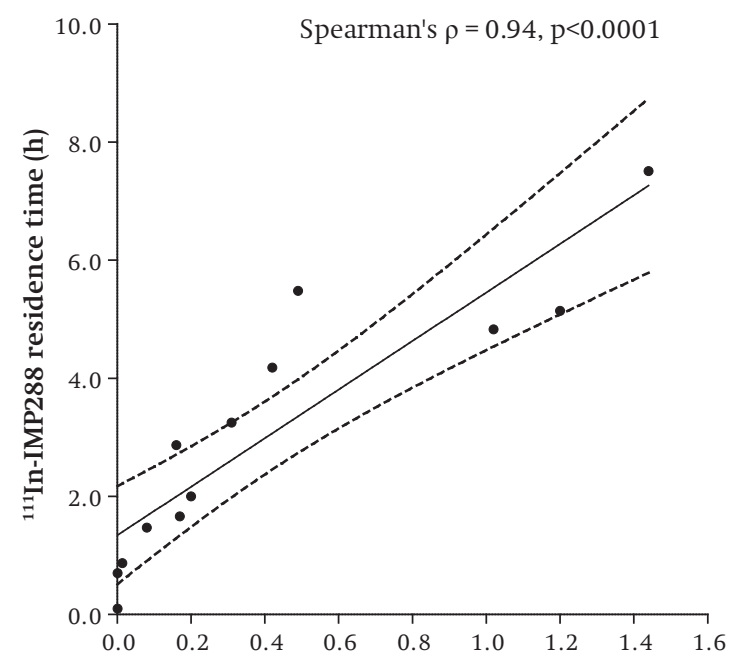

TF2 blood concentration $(\mu \mathrm{g} / \mathrm{mL})$

Figure 2 TF2 and IMP288 pharmacokinetics. A: TF2 serum concentration at the moment of IMP288 injection (individual patients). B: ${ }^{111}$ In-IMP288 residence times of the four cohorts (individual patients). C: Correlation between TF2 and IMP288 blood pharmacokinetics. Individual TF2 blood concentration at the moment of peptide injection plotted against the blood residence time of ${ }^{111}$ In-IMP288 (Spearman $\mathrm{r}=0.94, \mathrm{P}<0.0001$ ), with linear regression line (solid line) and the $95 \%$ confidence interval.

peptide dose (100 $\mu \mathrm{g}$ in cohort 1-3), IMP288 residence times correlated strongly with the TF2 blood concentrations at the time of peptide injection (Spearman's $\rho=$ $0.94, P<0.0001$ ) (Fig. 2 C).

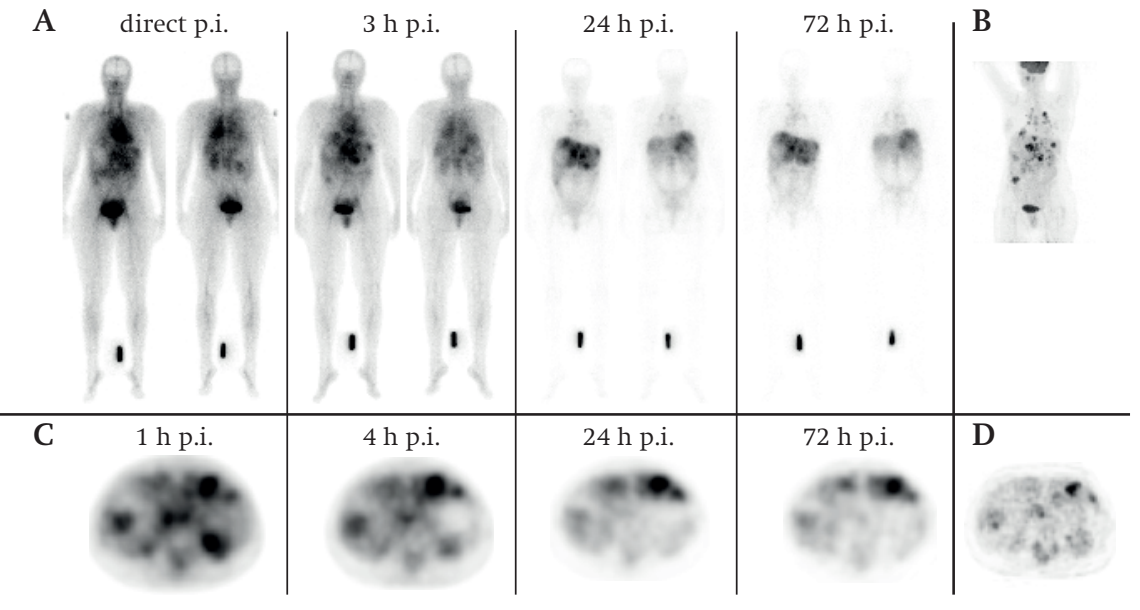

Figure 3 Whole-body planar scintigraphic images (A) of a patient with a primary colon tumor, liver, lung, lymph node (mediastinal), bone (vertebrae) and soft tissue (paravertebral) metastases, after injection of ${ }^{11} \mathrm{I} n-\mathrm{IMP} 288(185 \mathrm{MBq}, 100 \mu \mathrm{g})$, pretargeted with $150 \mathrm{mg}$ TF2 (anterior and poster, $10 \mathrm{~min}$ p.i., $3 \mathrm{~h}$ p.i., $24 \mathrm{~h}$ p.i., $72 \mathrm{~h}$ p.i.), and the corresponding FDG-PET (B). SPECT images of same patient (C), acquired directly after planar images (3D volume rendered, 1 h p.i., 4 h p.i., 24 h p.i., 72 h p.i.), and the corresponding FDG-PET (D).

\section{Scintigraphic imaging}

Tumor lesions were visualized as early as one hour after injection of the radiolabeled peptide in all patients, with ${ }^{111} \mathrm{In}$-scans and ${ }^{177} \mathrm{Lu}$-scans being highly congruent. Examples of whole-body planar and SPECT images of one of the patients are shown in Figure 3. ${ }^{111} \mathrm{In}$ - and ${ }^{177} \mathrm{Lu}-\mathrm{IMP} 288$ cleared from the blood and kidneys within 24 $\mathrm{h}$, with very limited kidney or any other normal tissue retention, resulting in high tumor-to-background ratios.

\section{Absorbed doses and individual ${ }^{177}$ Lu activity doses}

Kidney uptake of the radiolabeled peptide was relatively low. The predicted kidney absorbed doses ( $<0.50 \mathrm{mGy} / \mathrm{MBq}$ ) did not limit the maximum activity that could be administered; i.e., none of the patients would exceed the limit of $15 \mathrm{~Gy}$ to the kidneys with four cycles of $7.4 \mathrm{GBq}{ }^{177} \mathrm{Lu}-\mathrm{IMP} 288$.

The predicted radiation doses to the red bone marrow also were relatively low. Marrow doses increased in subsequent cohorts as the ratio of bsMAb to haptenpeptide was increased; i.e., the mean \pm SD of blood-based method for the 4 cohorts were: $0.008 \pm 0.003,0.034 \pm 0.011,0.045 \pm 0.028$, and $0.060 \pm 0.040$ (cohorts $1,2,3$ 
and 4, respectively, Fig. 4). However, the difference between marrow doses for cohort 1 vs cohorts 2-4 was the only significant finding ( $P=0.02$, Mann-Whitney, with Bonferonni correction). Individual patients in cohorts 2, 3, and 4 showed considerable variability in red marrow doses, which corresponded with the variable residence times for IMP-288 blood clearance within these cohorts. The low predicted red marrow doses indicated that high therapeutic doses of up to $7.4 \mathrm{GBq}$ could be administered (Table 1). In several patients of cohorts $2-4$, the ${ }^{177}$ Lu activity doses were reduced to keep the red marrow dose below 1.25 Gy in four successive cycles ( 0.31 Gy per treatment cycle), as required by the protocol. The results of two different methods that were used to estimate red marrow doses (blood- versus image-based method) were in good agreement (mean difference: $0.0040 \mathrm{mGy} / \mathrm{MBq}$, SD: $0.017 \mathrm{mGy} / \mathrm{MBq}$, Bland-Altman).

Patient 19 received the maximum allowed $7.4 \mathrm{GBq}$ of ${ }^{177} \mathrm{Lu}$-activity with $25 \mathrm{\mu g}$ IMP288. However, even though patients 20 and 21 also were allowed to receive 7.4 GBq on the basis of ${ }^{111}$ In-IMP288 assessment, poor labeling efficiency yielded only 5.6 GBq ${ }^{177} \mathrm{Lu}-\mathrm{IMP} 288$ for administration. The red marrow doses that were calculated after administration of ${ }^{177}$ Lu-IMP288 were in good agreement with the predicted absorbed doses from the ${ }^{111}$ In-IMP288 study. The Bland-Altman plot in Figure 5 shows a mean difference of $-0.0026 \mathrm{mGy} / \mathrm{MBq}$ (SD: $0.014 \mathrm{mGy} / \mathrm{MBq})$. As a consequence, the measured red marrow doses did not exceed the preset limit of 0.31 Gy in the majority of patients (11 out of 13 patients, $85 \%$ ), excluding cohort 1 , patients 20 and 21 from the analysis, as described above).

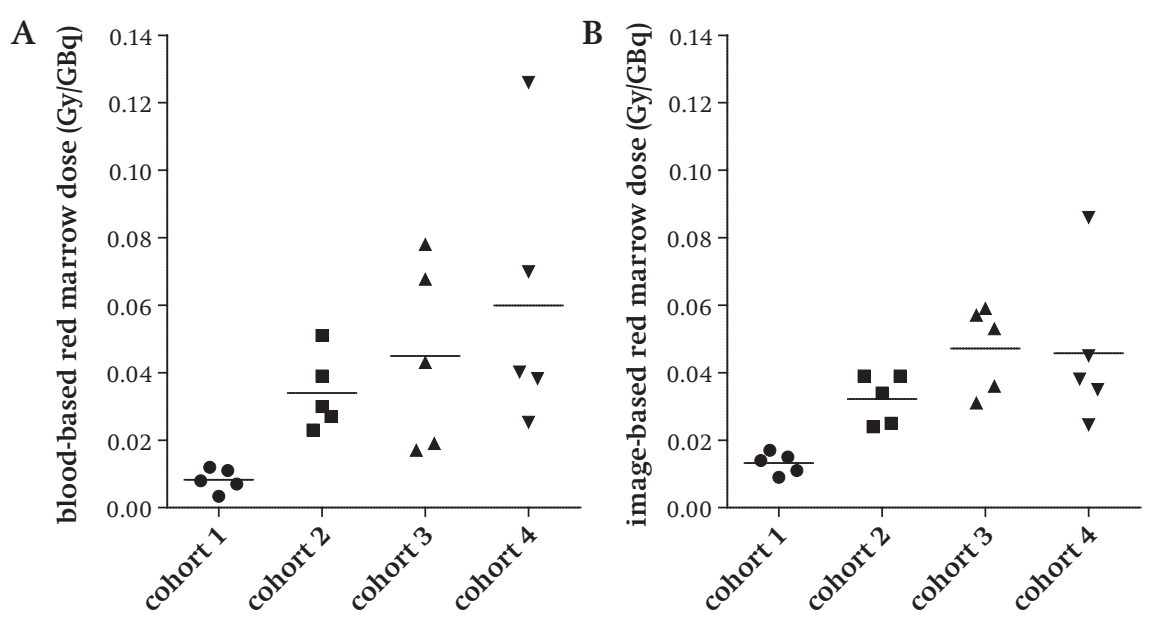

Figure 4 Simulated ${ }^{177} \mathrm{Lu}$ absorbed doses. The simulated ${ }^{177} \mathrm{Lu}-\mathrm{IMP} 288$ absorbed doses for red marrow, blood-based (A), and image-based (B) (individual patients in each cohort).

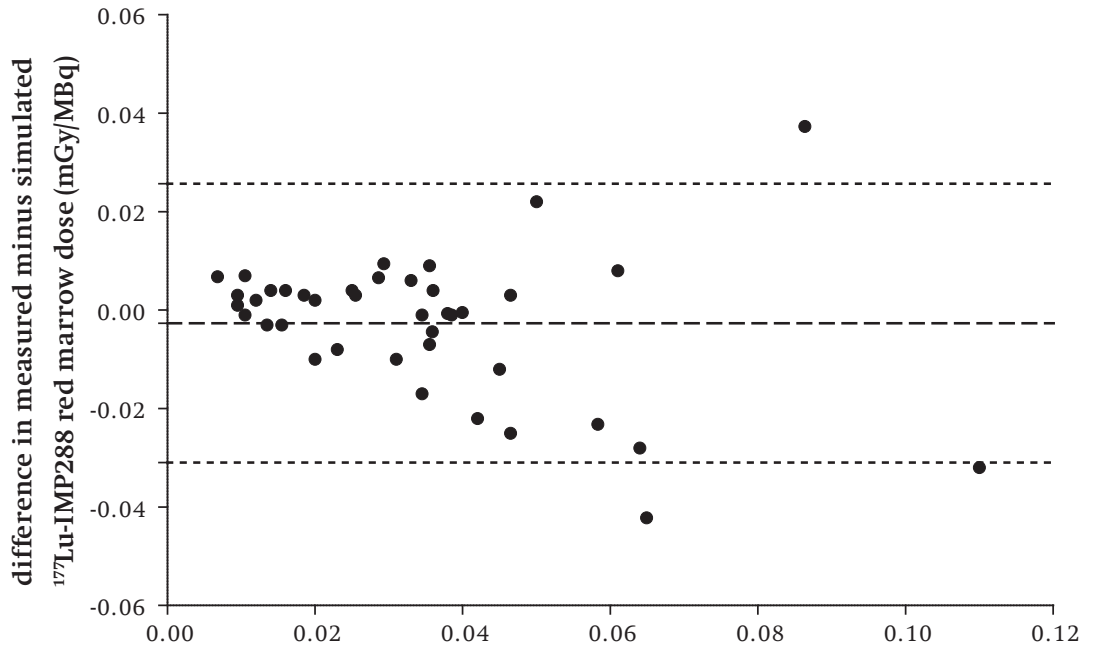

average of simulated and measured ${ }^{177} \mathrm{Lu}-\mathrm{IMP} 288$ red marrow dose (mGy/MBq)

Figure 5 Agreement between predicted and measured ${ }^{177} \mathrm{Lu}$ doses. Bland-Altman plot showing the difference between the measured minus the predicted data plotted against the average of both values. Dashed line is mean difference $(-0.0026 \mathrm{mGy} / \mathrm{MBq})$, dotted lines are $95 \%$ agreement limits $(-0.031$ and $0.026 \mathrm{mGy} / \mathrm{MBq})$.

\section{Toxicity}

None of the patients experienced non-hematological toxicity related to ${ }^{177} \mathrm{Lu}-\mathrm{IM} 288$. Hematologic toxicity was mild in most patients (grade $1-2$ in $30 \%$ of the patients). Two patients (10\%) had transient grade 3 or 4 hematologic toxicity: patient 10 (cohort 2) and patient 16 (cohort 3) had grade 3-4 thrombocytopenia and grade 3 lymphopenia. The nadir was 5-6 weeks after ${ }^{177} \mathrm{Lu}-\mathrm{IMP} 288$ administration, but both patients had complete recovery at 7-8 weeks p.i. without any complication or need for medical intervention. Remarkably, in those two patients, the ${ }^{177} \mathrm{Lu}$-absorbed doses were somewhat higher than predicted by the imaging study 0.45 and $0.48 \mathrm{~Gy}$ for patients 10 and 16, respectively), while the red marrow dose of all other patients did not exceed $0.31 \mathrm{~Gy}$ (resulting in only grade $0-2$ thrombocytopenia). The measured ${ }^{177} \mathrm{Lu}-\mathrm{IMP} 288$ absorbed doses correlated significantly with platelet toxicity (Spearman's $\rho=0.58, P=0.008$ ) (Figure 6).

\section{Therapeutic efficacy}

At evaluation by FDG-PET/CT-scan eight weeks after the first therapy cycle with TF2 and ${ }^{177} \mathrm{Lu}-\mathrm{IMP} 288$, all patients still had progressive disease. Therefore, none of the patients was eligible for a second therapy cycle. 


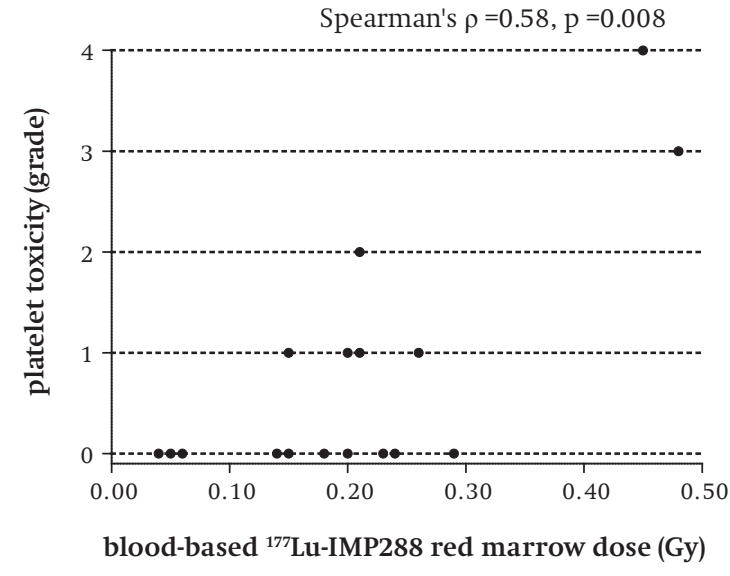

Figure 6 Correlation between ${ }^{177} \mathrm{Lu}$-absorbed doses and platelet toxicity. The measured ${ }^{177} \mathrm{Lu}-\mathrm{IMP} 288$ absorbed doses (calculated with blood-based method) correlated significantly with platelet toxicity (Spearman $\mathrm{r}=0.58, \mathrm{P}=0.008$ ).

\section{Discussion}

Patient-specific treatment planning in radioimmunotherapy is an attractive strategy to reduce the risk of hematologic toxicity. In this study, we showed that individualized dosing of PRIT with TF2 and ${ }^{17} \mathrm{Lu}$-IMP288 at fixed radiation-dose thresholds, estimated using a pre-therapeutic test dose of TF2 and ${ }^{111}$ In-IMP288, is feasible in patients with progressive metastatic CRC. Individualized activity dosing seemed essential, since relatively large inter-patient variations were observed in TF2 and IMP288 pharmacokinetics. We were able to identify those patients who were at the lowest risk for hematologic toxicity and who were eligible to receive the maximum activity dose of 7.4 GBq. At the same time, in those patients in whom the hapten-peptide blood and marrow residence time was somewhat slower in the pre-therapy cycle, the ${ }^{177} \mathrm{Lu}$-therapeutic activity doses could be reduced to prevent toxicity. As a consequence, red marrow radiation doses were low $(<0.5 \mathrm{~Gy})$. Of possible importance was the correlation between red marrow doses and hematological toxicity, where 2 patients who had the most significant hematological toxicity also had the highest red marrow dose. It was somewhat surprising that toxicity occurred at such low radiation doses, but we suspect that dose rate will need to be factored in future assessments, much like that found for renal dosimetry with radiolabeled peptides (27). The observed relation between the red bone marrow dose and hematological toxicity is in accordance with the results of some previous clinical radioimmunotherapy studies that demonstrated such a relationship $(20,28,29)$. On the other hand, various other studies reported a lack of correlation between dosimetric estimations and severity of toxicity (30-34). The discrepancy between the lack of correlation of the other studies and the correlation in this study might be due to differences in dose rate (35-38).

Two methods were employed to estimate the red marrow dose. The use of blood concentrations as a surrogate for bone marrow concentrations was permitted, because the pretargeting agents do not interact with any blood, marrow, or bone component, and metastatic bone involvement is limited in CRC. For imaging quantification, the cranium was used, because this region does not have overlapping organs, and because colorectal cancer frequently has pelvic and abdominal involvement that could mask the lumbar or sacral regions. Indeed, the lumbar vertebrae also are hindered by overlapping intestines (22). We showed that both dosimetry methods are feasible and provide similar results.

Our study showed some interesting features of the bsMAb and hapten-peptide pharmacokinetics. First, in all patients, the blood clearance of TF2 was much faste than that of similarly-sized IgG molecules (157 kD and $150 \mathrm{kD}$, respectively), which could be explained by the fact that TF2 lacks a $\mathrm{C}_{\mathrm{H}} 2$ domain, which enables recycling via FcRn-receptors (38). Our findings are in agreement with the observation that $\mathrm{C}_{\mathrm{H}} 2$ domain-deleted variants of IgG $(121 \mathrm{kDa})$ clear much faster from the blood than intact IgG (39). TF2's rapid blood clearance allowed us to reduce the interval between TF2 and IMP288 safely from five days to one. However, the short residence time of TF2 in the blood resulted in lower tumor uptake (40). Rapid blood clearance reduces the driving force of tumor accumulation. A bsMAb construct with a longer circulatory half-life, like IgGs, that would result in higher antibody-concentrations in the tumor could be advantageous in pretargeting.

Secondly, our data suggest that the inter-patient variability in IMP288 residence time in the blood could be explained by individual variations in bsMAb clearance rates, and consequently the bsMAb blood concentration at the time of peptide administration. If the concentration of circulating bsMAb is relatively high at the time the radiolabeled peptide is injected, a larger portion of the injected peptide dose will form immune complexes with the antibody in the circulation, which will increase the residence time of the radiolabeled peptide in the blood (and will thus increase the red marrow dose). These data are in accordance with the clinica observations of another phase I study, where patients with CEA-expressing tumors received an anti-CEA $\mathrm{x}$ anti-DTPA bsMAb in combination with an ${ }^{131}$ I-di-DTPAhapten (41).

The variation in TF2 blood clearance could be influenced by differences in individual baseline plasma CEA levels, because antigen-antibody complexes could be formed in serum, or by liver function, since the antibody is expected to be 
captured and degraded by this organ. However, no patient-specific characteristics could be identified to explain differences in bsMAb clearance rates (data not shown)

Three important pretargeting variables were examined in this study: (i) the interval between injection of the bsMAb and the peptide, (ii) the bsMAb dose, and (iii) the hapten-peptide dose (10). While the interval required for sufficient serum bsMAb clearance proved to be very short, tumor uptake of the radiolabeled peptide could be improved by administrating higher antibody doses. We also demonstrated that tumor uptake of the radiolabeled peptide increased at lower peptide doses. I remains to be established whether these parameters can be optimized further because at some point the residence time of the radiolabeled peptide will increase to levels that will cause increased red marrow toxicity.

No serious ${ }^{177}$ Lu-related non-hematological toxicity was observed, and only 2 cases of grade 3 and 4 hematological toxicity (thrombocytopenia) were encountered. Both of these patients had slightly higher red marrow doses in the ${ }^{177} \mathrm{Lu}$-study than predicted by the ${ }^{111}$ In-imaging procedure, which was not the case in the patient who had grade 0-2 hematologic toxicity. However, the overall differences in the total red marrow doses in these 2 patients were rather modest, and therefore it is likely that patient specific factors, such as age, prior myelotoxic treatments (e.g., prior radiation to bone marrow or chemotherapy), or extensive bone marrow involvement, also determined bone marrow toxicity. Both of these patients were $>70$ years of age (median age of the other patients was 63 years) and it is known that aging reduces the bone marrow reserve. They did not have bone marrow metastases, but both had received similar chemotherapeutic regimens and had similar interval between this study and their last treatment, as compared to the other patients, all of which could have contributed to their hematological toxicity.

${ }^{177} \mathrm{Lu}$ has several advantages over other $\beta$-emitting radionuclides, like ${ }^{90} \mathrm{Y}$ and ${ }^{131} \mathrm{I}$. The low energy of the beta particles with a short tissue penetration range is ideally suited for treatment of smaller-sized tumors. Its $11 \%$ abundance of photons allows scintigraphy to monitor the in vivo kinetics of the therapeutic administrations, and therefore we were able to compare directly the suitability of a pre-therapy imaging study with ${ }^{111} \mathrm{In}$ to the ${ }^{177} \mathrm{Lu}$ results. Unlike radioiodine, ${ }^{177} \mathrm{Lu}$ is a residualizing radionuclide, which means that the radionuclide is retained in tumor cells after internalization. Retention in the tumor, with rapid clearance from the norma tissues, also would benefit from ${ }^{177} \mathrm{Lu}$ 's longer half-life of 7 days. However, the half-life of ${ }^{90} \mathrm{Y}(64.1 \mathrm{~h})$ perhaps matched better with the residence time of IMP288 in the tumor, and its stronger beta emission would increase the radiation dose to the tumor. Another advantage of ${ }^{90} \mathrm{Y}$ is its longer tissue penetration range (maximum $12.0 \mathrm{~mm}$ ), an important factor when there is non-uniform distribution of uptake within the tumor, but also in this setting, where many patients had considerable tumor load. Therefore, future studies should consider using 90Y-labeled IMP288. In addition, the completion of the therapeutic regimen needs to be shortened, since in this trial, patients progressed after receiving only one-fourth of the planned therapeutic dose.

In conclusion, this study shows that pretargeted radioimmunotherapy in CEA-expressing CRC is feasible. Based on patient-specific dosimetry in a pre-therapy cycle with TF2 and ${ }^{111}$ In-IMP288, individualized, high therapeutic activity doses could be administered safely. Tumors are specifically and rapidly targeted with TF2 and ${ }^{177} \mathrm{Lu}-\mathrm{IMP} 288$, with limited hematological toxicity and no clinically-relevan non-hematological toxicity. Further clinical investigation should focus on therapeutic efficacy in patients with smaller volume disease and evaluating potential advantages of ${ }^{90} \mathrm{Y}$ over ${ }^{177} \mathrm{Lu}$ for labeling the peptide.

\section{Acknowledgements}

This work was supported by the Dutch Cancer Society (KWF Kankerbestrijding, grant no. KUN 2008-4038)

\section{References}

1. Baum RP, Rosch F. 1 World Congress on Ga-68 and Peptide Receptor Radionuclide Therapy (PRRNT), June 23-26, 2011, Zentralklinik Bad Berka, Germany. World J Nucl Med. 2011;10(1):1-2. Epub 2011/10/29. Goldenberg DM, Chang, C.H.,, Rossi, E.A., McBride, W.J, Sharkey, R.M. Pretargeted molecular imaging and radioimmunotherapy. Theranostics. 2012.

3. Schoffelen R, Boerman OC, Goldenberg DM, Sharkey RM, van Herpen CM, Franssen GM, et al. Development of an imaging-guided CEA-pretargeted radionuclide treatment of advanced colorecta cancer: first clinical results. Br J Cancer. 2013;109(4):934-42. Epub 2013/07/19.

4. Le Doussal JM, Martin M, GautherotE, Delaage M, BarbetJ. In vitro and in vivo targeting of radiolabeled monovalent and divalent haptens with dual specificity monoclonal antibody conjugates: enhanced divalent hapten affinity for cell-bound antibody conjugate. Journal of nuclear medicine. 1989;30(8):1358-66. Epub 1989/08/01.

5. Sharkey RM, Cardillo TM, Rossi EA, Chang CH, Karacay H, McBride WJ, et al. Signal amplification in

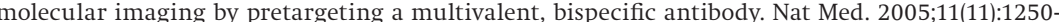
Epub 2005/11/01.

6. Griffiths GL, Chang CH, McBride WJ. Rossi EA, Sheerin A, Tejada GR, et al. Reagents and methods for PET using bispecific antibody pretargeting and 68Ga-radiolabeled bivalent hapten-peptide-chelete conjugates. Journal of nuclear medicine . 2004;45(1):30-9. Epub 2004/01/22. Koppe MJ, Bleichrodt RP, Soede AC, Verhofstad AA, Goldenberg DM, Oyen WJ, et al. Biodistributio and therapeutic efficacy of (125/131)I-, (186)Re-, (88/90)Y-, or (177)Lu-labeled monoclonal antibody MN-14 to carcinoembryonic antigen in mice with small peritoneal metastases of colorectal origin. Journal of nuclear medicine . 2004;45(7):1224-32. Epub 2004/07/06

8. McBride WJ, Zanzonico P, Sharkey RM, Noren C, Karacay H, Rossi EA, et al. Bispecific antibody pretargeting PET (immunoPET) with an 124I-labeled hapten-peptide. Journal of nuclear medicine. 2006:47(10):1678-88. Epub 2006/10/04

9. Schoffelen R, Sharkey RM, Goldenberg DM, Franssen G, McBride WJ, Rossi EA, et al. Pretargeted immuno-positron emission tomography imaging of carcinoembryonic antigen-expressing tumors 
with a bispecific antibody and a 68Ga- and 18F-labeled hapten peptide in mice with human tumor xenografts. Mol Cancer Ther. 2010;9(4):1019-27. Epub 2010/04/01

10. Schoffelen R, van der Graaf WT, Franssen G, Sharkey RM, Goldenberg DM, McBride WJ, et at. Pretargeted 177Lu radioimmunotherapy of carcinoembryonic antigen-expressing human colonic tumors in mice. Journal of nuclear medicine . 2010;51(11):1780-7. Epub 2010/11/06.

11. Sharkey RM, McBride WJ, Karacay H, Chang K, Griffiths GL, Hansen HJ, et al. A universal pretargeting system for cancer

12. Goldenberg DM, Rossi EA, Sharkey RM, McBride WJ, Chang CH. Multifunctional antibodies by the Dockand medicine . 2008;49(1):158-63. Epub 2007/12/14

13. Rossi EA, Goldenberg DM, Cardillo TM, McBride WJ, Sharkey RM, Chang CH. Stably tethered multiCo

14. Sharkey RM, Karacay H, Litwin S, Rossi EA, McBride WJ. Chang CH, et al. Improved therapeutic results by pretargeted radioimmunotherapy of non-Hodgkin's lymphoma with a new recombinant, trivalent nti-CD20, bispecific antibody. Cancer Res. 2008:68(13):5282-90. Epub 2008/07/03.

15. Schoffelen R, van der Graaf WT, Sharkey RM, Franssen GM, McBride WJ, Chang CH, et al. Quantitative immuno-SPECT monitoring of pretargeted radioimmunotherapy with a bispecific antibody in an intraperitoneal nude mouse model of human colon cancer. Journal of nuclear medicine. 2012:53(12):1926-32. Epub 2012/10/20.

16. Chang CH, Sharkey RM, Rossi EA, Karacay H, McBride W, Hansen HJ, et al. Molecular advances in pretargeting radioimunotherapy with bispecific antibodies. Mol Cancer Ther. 2002;1(7):553-63. Epub $2002 / 12 / 14$

17. Schoffelen R, van der Graaf WT, Sharkey RM, Franssen GM, McBride WJ, Chang CH, et al. Pretargeted mmuno-PET of CEA-expressing intraperitoneal human colonic tumour xenografts: a new sensitive detection method. EJNMMI Res. 2012;2(1):5. Epub 2012/01/31.

18. Sharkey RM, Karacay H, Vallabhajosula S, McBride WJ, Rossi EA, Chang CH, et al. Metastatic huma colonic carcinoma: molecular imaging with pretargeted SPECT and PET in a mouse model. Radiology. 008;246(2):497-507. Epub 2008/01/30.

19. Brouwers AH, Buijs WC, Mulders PF, de Mulder PH, van den Broek WJ, Mala C, et al. Radioimmunotherapy with [131I]cG250 in patients with metastasized renal cell cancer: dosimetric analysis an mmunologic response. Clin Cancer Res. 2005;11(19 Pt 2):7178s-86s. Epub 2005/10/06.

20. Stillebroer AB, Zegers CM, Boerman OC, Oosterwijk E, Mulders PF, O'Donoghue JA, et al. Dosimetric Analysis of 177Lu-CG250 Radioimmunotherapy in Renal Cell Carcinoma Patients: Correlation with Myelotoxicity and Pretherapeutic Absorbed Dose Predictions Based on 111In-cG250 Imaging. Journ of nuclear medicine . 2011. Epub 2011/12/14.

1. Sharkey RM, Rossi EA, McBride WJ, Chang CH, Goldenberg DM. Recombinant bispecific monoclona antibodies prepared by the dock-and-lock strategy for pretargeted radioimmunotherapy. Semin Nuc Med. 2010;40(3):190-203. Epub 2010/03/31

22. Visser E, Postema E, Boerman O, Visschers J, Oyen W, Corstens F. Software package for integrated data processing for internal dose assessment in nuclear medicine (SPRIND). Eur J Nucl Med Mol Imaging. 2007;34(3):413-21. Epub 2006/11/16.

23. Shen S, DeNardo GL, Sgouros G, O'Donnell RT, DeNardo S. Practical determination of patient-specific marrow dose using radioactivity concentration in blood and body. Journal of nuclear medicine. 1999;40(12):2102-6. Epub 2000/01/05

24. Forrer F, Krenning EP, Kooij PP, Bernard BF, Konijnenberg M, Bakker WH, et al. Bone marrow dosimetry in peptide receptor radionuclide therapy with [177Lu-DOTA(0),Tyr(3)]octreotate. Eur J Nuc Med Mol Imaging. 2009;36(7):1138-46. Epub 2009/02/28

25. Valkema R, Pauwels SA, Kvols LK, Kwekkeboom DJ, Jamar F, de Jong M, et al. Long-term follow-up of renal function after peptide receptor radiation therapy with (90)Y-DOTA(0),Tyr(3)-octreotide and (177) Lu-DOTA(0), Tyr(3)-octreotate. Journal of nuclear medicine . 2005;46 Suppl 1:83S-91S. Epub 2005/01/18.
26. Scala RJ. Biologic effects of ionizing radiation. In: P.J. Early BDS, editor. Principles and Practice of Nuclear Medicine. St Louis: Mosby; 1995. p. 123-7.

27. Barone R, Borson-Chazot F, Valkema R, Walrand S, Chauvin F, Gogou L, et al. Patient-specific dosimetry in predicting renal toxicity with (90)Y-DOTATOC: relevance of kidney volume and dose rate in findin a dose-effect relationship. Journal of nuclear medicine . 2005;46 Suppl 1:99S-106S. Epub 2005/01/18.

28. Juweid ME, Zhang CH, Blumenthal RD, Hajjar G, Sharkey RM, Goldenberg DM. Prediction of hematologic toxicity after radioimmunotherapy with (131)-labeled anticarcinoembryonic antigen monoclonal antibodies. Journal of nuclear medicine . 1999;40(10):1609-16. Epub 1999/10/16.

29. Vallabhajosula S, Goldsmith SJ, Hamacher KA, Kostakoglu L, Konishi S, Milowski MI, et al. Prediction of myelotoxicity based on bone marrow radiation-absorbed dose: radioimmunotherapy studies using 90Y- and 177Lu-labeled J591 antibodies specific for prostate-specific membrane antigen. Journal of nuclear medicine . 2005;46(5):850-8. Epub 2005/05/06.

30. Behr TM, Sharkey RM, Juweid ME, Dunn RM, Vagg RC, Ying Z, et al. Phase I/II clinical radioimmunotherapy with an iodine-131-labeled anti-carcinoembryonic antigen murine monoclonal antibody IgC. Journal of nuclear medicine . 1997;38(6):858-70. Epub 1997/06/01

31. Divgi CR, Bander NH, Scott AM, O'Donoghue JA, Sgouros G, Welt S, et al. Phase I/II radioimmunotherapy trial with iodine-131-labeled monoclonal antibody G250 in metastatic renal cell carcinoma. Clin Cancer Res. 1998;4(11):2729-39. Epub 1998/11/26.

32. O'Donoghue JA, Baidoo N, Deland D, Welt S, Divgi CR, Sgouros G. Hematologic toxicity in radio immunotherapy: dose-response relationships for I-131 labeled antibody therapy. Cancer Biothe Radiopharm. 2002;17(4):435-43. Epub 2002/10/25.

33. Vose JM, Wahl RL, Saleh M, Rohatiner AZ, Knox SJ, Radford JA, et al. Multicenter phase II study of iodine-131 tositumomab for chemotherapy-relapsed/refractory low-grade and transformed low-grade B-cell non-Hodgkin's lymphomas. J Clin Oncol. 2000;18(6):1316-23. Epub 2000/03/15.

34. Wiseman GA, White CA, Sparks RB, Erwin WD, Podoloff DA, Lamonica D, et al. Biodistribution an dosimetry results from a phase III prospectively randomized controlled trial of Zevalin radioimmunotherapy for low-grade, follicular, or transformed B-cell non-Hodgkin's lymphoma. Crit Rev Onco Hematol. 2001;39(1-2):181-94. Epub 2001/06/22.

35. Behr TM, Memtsoudis S, Sharkey RM, Blumenthal RD, Dunn RM, Gratz S, et al. Experimental studies on the role of antibody fragments in cancer radio-immunotherapy: Influence of radiation dose an dose rate on toxicity and anti-tumor enicacy. Int J Cancer. 1998;77(5):787-95. Eptb 1998/08/04.

6. Behr TM, Sharkey RM, Sgouros G, Blumenthal RD, Dunn RM, Kolbert K, et al. Overcoming the nephrotoxicity of radiometal-labeled immunoconjugates: improved cancer therapy administered to nude mouse model in relation to the internal radiation dosimetry. Cancer. 1997;80(12 Suppl):259 610. Epub 1997/12/24.

37. Howell RW, Goddu SM, Rao DV. Design and performance characteristics of an experimental cesium-137 irradiator to simulate internal radionuclide dose rate patterns. Journal of nuclear medicine. 1997;38(5):727-31. Epub 1997/05/01.

8. Ghetie V, Ward ES. FcRn: the MHC class I-related receptor that is more than an IgG transporter Immunol Today. 1997;18(12):592-8. Epub 1998/01/13.

39. Chinn PC, Morena RA, Santoro DA, Kazules T, Kashmiri SV, Schlom J, et al. Pharmacokinetics an tumor localization of (111)in-labeled HuCC49DeltaC(H)2 in BALB/c mice and athymic murine colo carcinoma xenograft. Cancer Biother Radiopharm. 2006;21(2):106-16. Epub 2006/05/19.

40. Jain RK. Physiological barriers to delivery of monoclonal antibodies and other macromolecules in tumors. Cancer Res. 1990;50(3 Suppl):814s-9s. Epub 1990/02/01.

41. Kraeber-Bodere F, Faivre-Chauvet A, Ferrer L, Vuillez JP, Brard PY, Rousseau C, et al. Pharmacokinetic and dosimetry studies for optimization of anti-carcinoembryonic antigen $\mathrm{x}$ anti-hapten bispecific antibody-mediated pretargeting of lodine-131-labeled hapten in a phase I radioimmunotherapy trial. Clin Cancer Res. 2003;9(10 Pt 2):39735-815. Epub 2003/09/25. 


\section{3}

Tumor and red bone marrow dosimetry: Comparison of methods for prospective treatment planning in pre-targeted radioimmunotherapy

Wietske Woliner - van der Weg, Rafke Schoffelen, Robert F. Hobbs, Martin Gotthardt, David M. Goldenberg, Robert M. Sharkey, Cornelis H. Slump, Winette T.A. van der Graaf, Wim J.G. Oyen, Otto C. Boerman, George Sgouros, Eric P. Visser 


\section{Abstract}

Red bone marrow (RBM) toxicity is dose-limiting in (pretargeted) radioimmunotherapy (RIT). Previous blood-based and two-dimensional (2D)-image-based methods have failed to show a clear dose-response relationship. We developed a three-dimensional (3D)-image-based RBM dosimetry approach using the Monte Carlo-based 3D-Radiobiological Dosimetry (3D-RD) software, and determined its additional value for predicting RBM toxicity.

Methods: RBM doses were calculated for thirteen colorectal cancer patients after pretargeted RIT with the two-step administration of an anti-CEA $\mathrm{x}$ anti-HSC bispecific monoclonal antibody and a ${ }^{177} \mathrm{Lu}$-labeled di-HSG-peptide. 3D-RD RBM dosimetry was based on lumbar vertebrae, delineated on SPECT scans acquired directly, 3, 24 and $72 \mathrm{~h}$ after ${ }^{177} \mathrm{Lu}$ administration. RBM doses were correlated to hematologic effects, according to NCI-CTC v3, and compared with conventiona 2D-cranium-based and blood-based dosimetry results. Tumor doses were calculated with 3D-RD, which has not been possible with 2D dosimetry. Tumor-to-RBM dose ratios were calculated and compared for ${ }^{177} \mathrm{Lu}$-based-pretargeted RIT and simulated pretargeted RIT with ${ }^{90} \mathrm{Y}$.

Results: 3D-RD RBM doses of all seven patients who developed thrombocytopenia were higher (range 0.43-0.97 Gy) than of the six patients without thrombocytopenia (range 0.12-0.39 Gy), except in one patient (0.47 Gy) without thrombocytopenia, but with grade 2 leucopenia. Blood and 2D-image-based RBM doses for patients with grade 1-2 thrombocytopenia were in the same range as in patients without thrombocytopenia (0.14-0.29 Gy and 0.11-0.26 Gy, respectively). Blood-based RBM doses for two grade 3-4 patients were higher (0.66 Gy and 0.51 Gy, respectively) than the others, and the cranium-based dose of only the grade 4 patient was higher (0.34 Gy). Tumor-to-RBM dose ratios would increase by $25 \%$ on average when treating with ${ }^{90} \mathrm{Y}$ instead of ${ }^{177} \mathrm{Lu}$.

Conclusion: 3D dosimetry identifies patients at risk of developing any grade of RBM toxicity more accurately than blood- or 2D-image-based methods. It has the added value to enable calculation of tumor-to-RBM dose ratios.

\section{Introduction}

The aim of radioimmunotherapy (RIT) is to selectively target radioactivity to tumor lesions, with limited radiation dose to healthy tissues. The absorbed dose (AD) depends on the patient-specific pharmacokinetics of the tracer, the administered activity and the radionuclide. After pre-therapeutic administration of a diagnosticlabeled compound, dosimetric calculations (matched pair dosimetry) lead to a patientspecific insight into how best to treat the patient. For example, pre-treatment dosimetry can be used to adjust the individual therapy dose, or even be used to select the mos suitable radionuclide for therapy. Ideally, this will lead to an improved benefitversus-risk ratio for individual patients.

In external beam radiotherapy, patient-specific treatment planning is common practice, because treatment planning is based on absorbed dose distributions an dose-response relationships for both tumor and normal tissue are relatively well known. However, for RIT, dose-response relationships have not been rigorously established and dose estimation has been less accurate and focused on calculating mean absorbed doses. In radionuclide therapy, the mean absorbed dose may not be useful for predicting tumor response and, in some cases, for predicting normal organ toxicity. Therefore, further investigation of dose-response relationships and development of dosimetry methods that provide dose-volume histograms and that incorporate radiobiological modeling for the clinical practice of radioimmunotherapy are highly desirable.

Since the red bone marrow (RBM) is often dose-limiting in RIT (1-6), the focus of the present study was on RBM dose calculation and the dose-toxicity relationship. Commonly used methods to calculate the RBM AD are the blood-based method, two-dimensional (2D) image-based method, or a combination of these two methods. Although blood-based (or partially blood-based) dosimetry is an accepted method for estimation of the RBM dose $(1,3,7-9)$, the correlation with the observed hematological toxicity is insufficient for clinical use $(5,10)$. Especially when the radiopharmaceutical shows RBM retention, 2D image-based dosimetry seems to be a better predictor for hematological toxicity than the blood-based method (5).

In our institution, both the above-mentioned methods were prospectively applied in a clinical phase I pretargeted RIT study in twenty patients with advanced colorectal cancer (11). Dosimetric data based on administration of a diagnostic

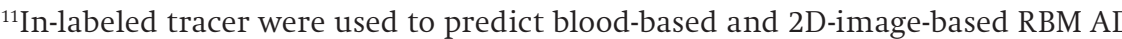
in a subsequent treatment with the same compound, but labeled with ${ }^{177} \mathrm{Lu}$. Although relatively low RBM absorbed doses ( $<0.31 \mathrm{~Gy}$ ) were predicted in several patients, RBM toxicity was observed. According to the NCI Common Terminology Criteria v3, five patients developed grade 1-2 and two patients grade 3-4 thrombo- 
cytopenia, and three patients developed grade 1-2 leucopenia. Based on the applied dosimetry methods, no dose limit based on the ${ }^{177} \mathrm{Lu}$ dosimetry could be defined that distinguishes the patients who showed toxicity from those without toxicity. Clearly, these calculations did not reliably predict toxicity in this experimenta treatment. This indicates that an improved dosimetry method leading to doses correlating with the RBM toxicity and probably in the future useful for prediction, -and ideally prevention- of toxicity is desirable.

Furthermore, based on the data of the phase I study, it was suggested that treatment with ${ }^{90} \mathrm{Y}$ might improve the tumor-to-RBM dose ratio (11). The half-life of ${ }^{90} \mathrm{Y}(2.66$ days versus 6.71 days for ${ }^{177} \mathrm{Lu}$ ) better corresponds with the peptide residence time in the tumor (11), and therefore should lead to a higher tumor-to-RBM ratio. In addition, ${ }^{90} \mathrm{Y}$ is a well-known therapeutic radionuclide, e.g., as ${ }^{90} \mathrm{Y}$-ibritumomab tiuxetan (Zevalin ${ }^{\circledR}$, Bayer Schering Pharma AG), an FDA-approved drug for treatment of non-Hodgkin's lymphoma.

Assessment of the tumor-to-RBM dose ratio requires 3D dosimetry, since 2D mage-based tumor dosimetry does not lead to reliable results as a result of overprojection with background tissue. Also, for the RBM dose calculation, 3D dosimetry is preferred since it has been shown that single photon emission computed tomography (SPECT)-based dosimetry leads to smaller errors than planar imagebased dosimetry (12). This is mainly due to the lack of overprojecting organs and more accurate attenuation correction.

Therefore, we hypothesized that 3D SPECT-based RBM dosimetry results better correlate with bone marrow toxicity, than the blood-based and 2D image-based dosimetry results.

Recently, Boucek and Turner demonstrated a relationship between myelotoxicity and RBM dose, calculated using a 3D-image-based dosimetry method in 23 patients with non-Hodgkin lymphoma. They used a three compartment model, comprising the blood, whole body and RBM activity, which was measured in the spine, pelvis and femur (13). They calculated the RBM dose with a method relying on S-values, which are calculated using idealized representative model-based anatomical phantoms (14). The use of idealized model-based S-values for individual patient anatomy may not account for important differences between the model-based representation and the individual patient anatomy. Such differences are particularly important in a therapeutic setting. Schwartz et al. (4), who also used an S-value based method, did not find a correlation with toxicity. This emphasizes the potential importance of investigating dosimetry methods for RBM dosimetry that do not rely on idealized anatomical models and that are more likely to give results that correlate with RBM toxicity.
In this study, we applied a method for 3D RBM dosimetry that does not depend on S-values, but instead uses Monte Carlo simulations for the dose calculation. Fo this purpose, the 3D Radiobiological Dosimetry (3D-RD) software $(15,16)$ was used in patients who had undergone pretargeted RIT with a bispecific antibody and ${ }^{177} \mathrm{Lu}$-labeled peptide. The aims were: (I) to accurately estimate the RBM dose and the tumor dose; (II) to correlate the RBM dose with the RBM toxicity; and (III) to use the results for selection of the most suitable radionuclide for this therapy.

\section{Materials and Methods}

\section{Patients}

Patients with metastatic colorectal cancer for whom no standard treatment was available were eligible for the pretargeted RIT study. The protocol (ClinicalTrials. gov Identifier NCT00860860) was approved by the Regional Ethics Review Board (CMO). Written confirmed consent was obtained from all patients prior to any study-related procedures.

The patients received the anti-CEACAM5 $\mathrm{x}$ anti-hapten humanized trivalen bispecific antibody TF2. One day later the ${ }^{177} \mathrm{Lu}$-labeled di-HSG peptide IMP288 (2.5-7.4 GBq) was administered as reported previously (11). Of the 20 patients in this study, 13 were eligible for the 3D image based dosimetry as they had undergone a series of four SPECT scans after administration of the ${ }^{177}$ Lu-labeled peptide.

\section{Image acquisition and use in dosimetry}

Anterior and posterior whole body planar images were acquired immediately (at 8 $\mathrm{cm} / \mathrm{min}$ scan speed), at 3 hours $(6 \mathrm{~cm} / \mathrm{min})$, at 24 hours and at 72 hours (both $4 \mathrm{~cm} /$ min) after administration of the ${ }^{177} \mathrm{Lu}$-labeled peptide, and followed by a SPECT scan involving continuous, circular scanning with a $180^{\circ} \mathrm{scan}$ arc, 64 views with both camera heads and 19s/view. For each patient, a region for the SPECT scanning was selected that contained the kidney region and at least one tumor lesion, as well as at least two lumbar vertebrae (LV).

A Siemens dual-head gamma camera (ECAM, Hoffmann Estates, IL), equipped with medium energy collimators was used with a symmetric 15\% window over the $208 \mathrm{keV}$ energy peak for SPECT scanning, and an additional 15\% window over the $113 \mathrm{keV}$ peak for the planar images.

The planar ${ }^{177} \mathrm{Lu}$ images were only used to calculate the ${ }^{177} \mathrm{Lu}$ RBM dose. Due to overprojection of background tissue, tumor dosimetry could not be performed on planar images.

Subsequently, the SPECT scans were used for tumor and RBM dosimetry of the ${ }^{77} \mathrm{Lu}$ dose. Simulation of ${ }^{90} \mathrm{Y}$ doses was performed (matched pair dosimetry) for 
comparison with the ${ }^{177} \mathrm{Lu}$ tumor-to-RBM dose ratios. For the ${ }^{90} \mathrm{Y}$ simulation, the SPECT images were rescaled to correct for the difference in half-life. Absorbed doses were calculated relative to the administered activity.

A contrast-enhanced diagnostic CT scan and a FDG-PET/CT (Biograph BGO duo, Siemens Medical Solutions, Knoxville, TN, USA) in accordance with the EANM guidelines were performed within two weeks prior to study entry, and for follow up, eight weeks after ${ }^{177} \mathrm{Lu}$ injection (17)

\section{D image and blood-based red bone marrow dosimetry}

During the pretargeted RIT study, the dose to RBM was calculated using a blood-based (3) and a 2D image-based dosimetry method (18).

The blood-based RBM dose was based on blood samples collected 2 minutes, 30 minutes, 1, 2, 4, 24 and $72 \mathrm{~h}$ after peptide injection and on the total body activity (retrieved from the whole body planar images). The blood samples were counted in a gamma counter (Wizard, Pharmacia-LKB, Sweden), using appropriate energy windows, with reference samples prepared from the injected products. Whole body activities were calculated and combined with the blood counts for the final dose calculation as described by Shen et al. (3) This was performed with the SPRIND software (18) using an RBM-to-blood activity concentration of 1, as was determined for ${ }^{177}$ Lu-peptide (19).

For the 2D image-based method, SPRIND was used to delineate the cranium, representing the activity in the RBM and to calculate the time-integrated activity coefficients. The time-integrated activity coefficient of the cranium activity was divided by the fraction of the RBM mass in the cranium to the RBM mass in the total skeleton, for which the default value of 0.119 was taken from ICRP23's Reference Man. Subsequently, OLINDA software (20) was used to calculate the RBM dose

\section{SPECT image reconstruction}

SPECT scans were reconstructed using the iterative reconstruction software ReSPECT (Scivis, Germany), in six iterations, without noise reduction, with background subtraction and attenuation correction using an attenuation coefficient of $0.13 \mathrm{~cm}^{-1}$, based on the results of Brown et al. (21). The algorithm uses attenuation correction with a constant attenuation coefficient within the body contours estimated from photo-peak and scatter projections.

This implies that a correct body contour is needed for quantitative evaluation of the images. In ReSPECT, the 'threshold object background' is used for definition of the body contour; voxels with a value higher than this threshold are assigned to the body. The contour can be defined correctly by the default threshold as long as the target (body)-to-background activity ratio is high enough, which was the case for the first two SPECT scans. For the third and fourth scans, the contour was fitted to the contour of the first scan by lowering the threshold value, as the lower whole body activity would have led to a smaller body contour at the default settings. The comparison of body contours of the early and later SPECT scans was performed with MATLAB (MathWorks, Natick, Massachusetts, U.S.A)

\section{Calibration}

To convert the pixel values in the SPECT images to activity (Bq), a calibration experiment was performed. A cylindrical phantom was filled with $440 \mathrm{MBq}{ }^{177} \mathrm{Lu}$ dissolved in $9.1 \mathrm{~L}$ water, and imaged using the same scanning and reconstruction protocol as used for the patient-SPECTs. The known activity at the time of acquisition, combined with the number of counts in a volume drawn around the phantom, resulted in a calibration factor of $6.23 \times 10^{-6} \mathrm{MBq}$ per count. In a separate calculation we estimated the dead time typically present in patient scans and in the phantom scan, using the equations that hold for paralyzable system as described in Cherry et al (22). The observed values based on quality control measurements of the SPECT camera, using the "two-source method", were in the order of $0.5-2 \mu$ s. This led to errors in activity concentrations of at maximum $1 \%$. For this reason we did not correct the images for dead time.

\section{Delineation and co-registration}

Tumor-oriented co-registration of the SPECT and the low-dose CT images was performed using the HERMES Gold 2.10 software (HERMES Medical Solutions, Stockholm, Sweden). The co-registered CT images were cropped and scaled to the same matrix size $(128 \times 128 \times 78)$ and voxel dimensions $(4,795 \times 4.795 \times 4.795 \mathrm{~mm})$ as the SPECT images. For each patient, VOIs were manually delineated in the LV $\left(\mathrm{VOI}_{\mathrm{LV}}\right)$ on the SPECT images for RBM dosimetry, and for one tumor lesion ( $\mathrm{VOI}_{\mathrm{tumor}}$ on the co-registered CT images for the tumor dosimetry.

For the $\mathrm{VOI}_{\mathrm{IV}}$, at each time point, the RBM containing parts of at least two LV were delineated and further processed as one VOI. If delineation on the SPECT image was not possible because the LV could not be distinguished from the background, the co-registered CT image was used for delineation.

Tumors were delineated on the rescaled CT by using a SPECT overlay. Occasionally, after checking the VOIs on the PET images, the regions were changed so that FDG-positive areas of tumors that were SPECT-negative were included in the delineation, because FDG-PET and SPECT images show different aspects of the pathophysiology. Necrotic cores were included in the VOI, since these areas might still contain vital tumor cells. 
3D-RD

For the 3D dosimetry, the Monte Carlo-based 3D-RD dosimetry package -developed at the Johns Hopkins Medical Institute (Baltimore, USA)- was used $(16,23)$. In thi software, the co-registered low-dose CT scan is used to assign a density value and composition (soft tissue, lung or bone) to each voxel in the corresponding SPECT scans. The density, composition and the activity maps were the input for the Mont Carlo (MC) simulations. For each SPECT scan, $10^{6} \mathrm{MC}$ simulations were run, using the spectra probability distributions obtained from MIRD (24), resulting in a dose rate value per voxel for each scan.

The dose per voxel was calculated by integrating the dose rates for each voxel, using a hybrid trapezoidal-exponential fit consisting of linear fits between the first three time points and an exponential tail obtained by fitting to the final two time points (25). This finally resulted in a dose map. The VOI mean absorbed doses were obtained by summing the deposited energy in the VOIs, and integrating these resulting VOI dose rates. For this integration a similar fit as for the voxel doses was used.

$3 \mathrm{D}$ tumor dosimetry - For the $\mathrm{VOI}_{\text {tumor }}$, the mean absorbed dose $\left(\mathrm{AD}_{\mathrm{tumor}}\right)$ and a dose volume histogram (DVH) were calculated using 3D-RD. The DVH provides insight into the spatial distribution of $\mathrm{AD}$ in the tumor. For comparison with the $\mathrm{AD}$ the $\mathrm{AD}$ in $\mathrm{VOI}_{\text {tumor }}$ was also calculated using the sphere model as incorporated in OLINDA (20). For this purpose, the mass of the VOI was calculated using the density map created from the low-dose CT and was used as the sphere mass as required by OLINDA. The tumor time-integrated activity coefficient was calculated using the total activity in the $\mathrm{VOI}_{\text {tumor }}$ at the four different time points, again using a similar hybrid trapezoidal-exponential fit.

3D LV-based RBM dosimetry - The rigid co-registration as described above was tumororiented, and it was observed that this did not lead to adequate co-registration of the LV, especially when slight differences in bending of the spine were observed. Therefore, delineation of the LV VOI was performed on each SPECT image separately, precluding the ability to perform voxelized dosimetry and thus only mean dose rates and mean absorbed doses were calculated for the $\operatorname{VOI}_{\mathrm{LV}}\left(\mathrm{AD}_{\mathrm{RBM}}\right)$

In addition to $\mathrm{AD}_{\mathrm{RBM}}$, the biological effective dose $\left(\mathrm{BED}_{\mathrm{RBM}}\right)$ was also calculated:

$$
B E D_{R B M}=A D_{R B M}\left(1+\frac{G(\infty)}{\alpha / \beta} A D_{R B M}\right)
$$

with $\alpha$ and $\beta$ the radiobiological parameters from the linear quadratic equation model $(\alpha / \beta=10 \mathrm{~Gy}(26)[26])$, AD the absorbed dose and $G(\infty)$ the Lea-Catcheside $G$-factor expressing the reduction in cell kill as a result of sublethal damage repair during treatment and which depends on the DNA repair rate $(\mu): 0.46 \mathrm{~h}^{-1}(26)$.

The 3D LV-based $\mathrm{AD}_{\mathrm{RBM}}$ was compared to the blood-based and the $2 \mathrm{D}$ craniumbased RBM dosimetry results, and correlated with the grade of thrombocytopenia graded according to NCI Common Terminology Criteria v3), since thrombocytopenia was the major toxicity experienced in this study.

\section{Tumor-to-RBM dose ratios}

In adequate treatment settings, the $\mathrm{AD}$ should be sufficiently higher than the $A D_{R B M}$. To evaluate this, the tumor-to-RBM dose ratio $A D_{\text {tumor }} / A_{\text {RBM }}$ was calculated. This calculation was done for the ${ }^{177} \mathrm{Lu}$ dose and the simulated ${ }^{90} \mathrm{Y}$ dose.

\section{Results}

\section{Patients, VOIs and RBM toxicity}

Table 1 summarizes patient and VOI characteristics, the calculated mean tumor and RBM doses and the grades of thrombocytopenia and leucopenia, representing the severity of the RBM toxicity. Patient numbers are the same as those used in the report of the phase 1 study (11). Figure 1 shows an example of a tumor lesion in the liver on CT, SPECT and PET images.

\section{D tumor doses}

As shown in Table 1, the ${ }^{177} \mathrm{Lu} \mathrm{AD}_{\text {tumor }}$ are 0.46 to $4.52 \mathrm{~Gy}$. A typical DVH of a tumor is shown in Figure 2 . The $\mathrm{AD}_{\text {tumor }}$ calculated with $3 \mathrm{D}-\mathrm{RD}$ was systematically greater than the OLINDA $\mathrm{AD}$, although in most patients the difference was small (difference $<5.0 \%$ ), except for one patient (number 10) who showed a 9.6\% higher 3D-RD tumor dose.

In all patients the BED was just slightly higher than the $\mathrm{AD}$ with a maximum difference of $0.11 \%$, which is expected due to the relatively low AD values.

\section{Correlation between RBM dose and RBM toxicity}

Figure 3 shows the blood-based, cranium-based and 3D-RD-based RBM doses vs. the grade of thrombocytopenia. 3D-RD-based RBM doses (Figure 3A) were generally higher than the doses calculated with the blood-based and 2D-image-based methods (Figures $3 \mathrm{~B}$ and $3 \mathrm{C}$ respectively). The highest 3D-RD-based RBM dose without thrombocytopenia and/ or leucopenia was 0.39 Gy. The other patient showed RBM toxicity and had RBM doses in the range of 0.42-0.97 Gy. There was 
Table 1 Patient, and VOItumor details and dose results

\begin{tabular}{|c|c|c|c|c|c|c|c|c|c|}
\hline \multirow[t]{2}{*}{ pt. \# } & \multirow{2}{*}{$\begin{array}{l}\text { Age } \\
\text { (y) }\end{array}$} & \multirow[t]{2}{*}{ Gender } & \multirow{2}{*}{$\begin{array}{l}\text { Location } \\
\text { VOI }_{\text {tumor }}\end{array}$} & \multirow{2}{*}{$\begin{array}{l}\mathrm{AA} \\
\text { (GBq) }\end{array}$} & \multirow{2}{*}{$\begin{array}{l}\text { Volume } \\
\text { VOI }_{\text {tumor }} \\
(\mathrm{mL})\end{array}$} & \multirow[t]{2}{*}{ tox } & \multicolumn{2}{|l|}{ 3D-RD } & \multirow{2}{*}{$\begin{array}{l}\text { OLINDA } \\
\mathrm{AD}_{\text {tumor }} \\
\text { (Gy) }\end{array}$} \\
\hline & & & & & & & $\begin{array}{l}\mathrm{AD}_{\text {tumor }} \\
(\mathrm{Gy})\end{array}$ & $\begin{array}{l}\mathrm{AD}_{\mathrm{RBM}} \\
\text { (Gy) }\end{array}$ & \\
\hline 7 & 63 & $\mathrm{~F}$ & Rectum & 6.2 & 14 & $0-0$ & 0.73 & 0.39 & 0.71 \\
\hline 10 & 70 & M & Liver & 7.4 & 70 & $4-1$ & 2.94 & 0.51 & 2.67 \\
\hline 11 & 55 & F & Liver & 7.4 & 44 & $0-0$ & 2.53 & 0.29 & 2.47 \\
\hline 12 & 70 & M & Liver & 7.4 & 386 & $0-0$ & 1.54 & 0.30 & 1.47 \\
\hline 13 & 76 & M & Colon & 7.4 & 23 & $0-0$ & 0.51 & 0.12 & 0.51 \\
\hline 14 & 52 & F & Liver & 4.0 & 23 & $1-0$ & 3.70 & 0.70 & 3.72 \\
\hline 15 & 58 & M & Liver & 5.9 & 285 & $1-0$ & 0.86 & 0.58 & 0.83 \\
\hline 16 & 76 & M & Colon & 4.6 & 121 & 3-2 & 0.66 & 0.97 & 0.64 \\
\hline 17 & 73 & $\mathrm{M}$ & Lung & 4.5 & 65 & $2-0$ & 0.63 & 0.43 & 0.62 \\
\hline 18 & 63 & F & Liver & 2.5 & 104 & $0-2$ & 4.52 & 0.47 & 4.44 \\
\hline 19 & 66 & F & Liver & 7.4 & 141 & $0-0$ & 1.45 & 0.28 & 1.38 \\
\hline 20 & 72 & M & Lung & 5.6 & 723 & $1-0$ & 0.46 & 0.72 & 0.45 \\
\hline 21 & 39 & $\mathrm{~F}$ & Liver & 5.6 & 154 & $0-0$ & 2.47 & 0.23 & 2.45 \\
\hline Mean & 64 & & & & 166 & & 1.77 & 0.46 & 1.72 \\
\hline
\end{tabular}

$\mathrm{RBM}=$ red bone marrow $\mathrm{AA}$ = administered activity, pt \# = patient number, tox = toxicity: grade thrombocytopenia - grade leucopenia

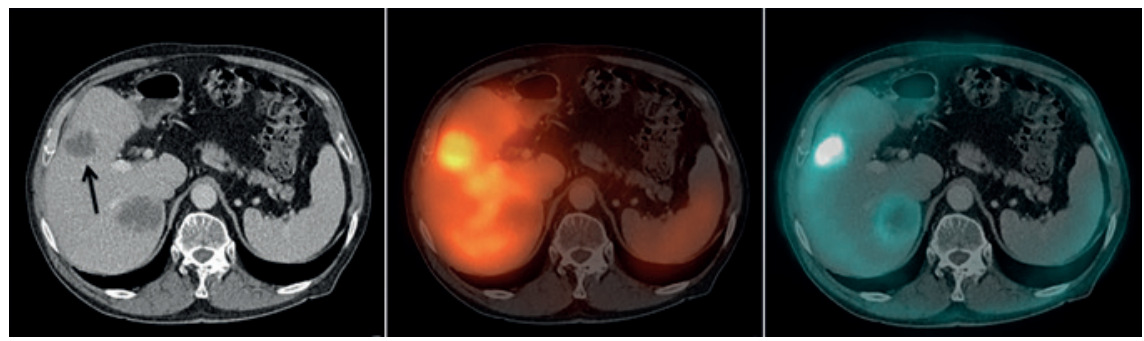

Figure 1 Metastases in the liver of patient 10 showing the lesions on CT (left), ${ }^{17} \mathrm{Lu}$-peptide SPECT/CT (middle) and FDG-PET/CT (right). The arrow indicates the lesion that was used for the tumor dosimetry.

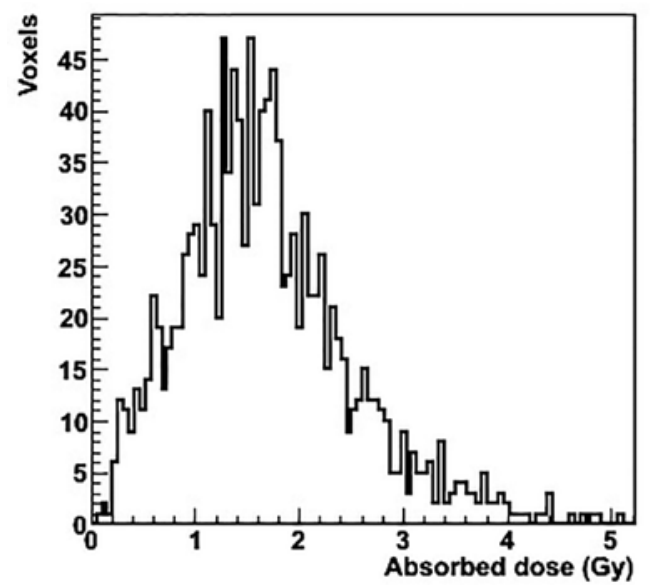

Figure 2 Dose volume histogram of the tumor volume of interest, located in the liver of patient 19.

only one patient in this range $(0.47 \mathrm{~Gy})$ without thrombocytopenia, but this patient had a grade 2 leucopenia. As shown in Figures 3 B and C, the blood-based and cranium-based methods do not separate patients with and without any grade of toxicity. Based on the blood-based RBM dose, only patients with grade 3-4 thrombocytopenia could be distinguished $(0.51$ and $0.66 \mathrm{~Gy})$ from the others patients (0.14-0.29 Gy). For the cranium-based dosimetry, the patient with grade 4 thrombocytopenia was the only patient with toxicity who showed a dose outside the AD range of the patients without toxicity (0.34 Gy vs $0.11-0.26$ Gy for the others). Some patients showed accumulation of activity in the RBM (see figure 4), leading to a higher RBM dose.

\section{Tumor to RBM dose ratios for ${ }^{177} \mathrm{Lu}$ compared to ${ }^{90} \mathrm{Y}$}

The effect of ${ }^{90} \mathrm{Y}$ versus ${ }^{177} \mathrm{Lu}$ was shown by the simulated ${ }^{90} \mathrm{Y}$ dosimetry (see Table 2). The mean increase in the tumor to RBM dose ratio is $25 \%$ when using ${ }^{90} \mathrm{Y}$ instead of ${ }^{177} \mathrm{Lu}$, and the mean ratio for the group changed from 4.68 for ${ }^{177} \mathrm{Lu}$ to $5.41 \mathrm{for}{ }^{90} \mathrm{Y}$. Interestingly, patients with a high RBM dose in ${ }^{177} \mathrm{Lu}$ treatment showed the largest percentage increase in tumor-to-RBM dose ratio when changing to ${ }^{90} \mathrm{Y}$. (Figure 5) 

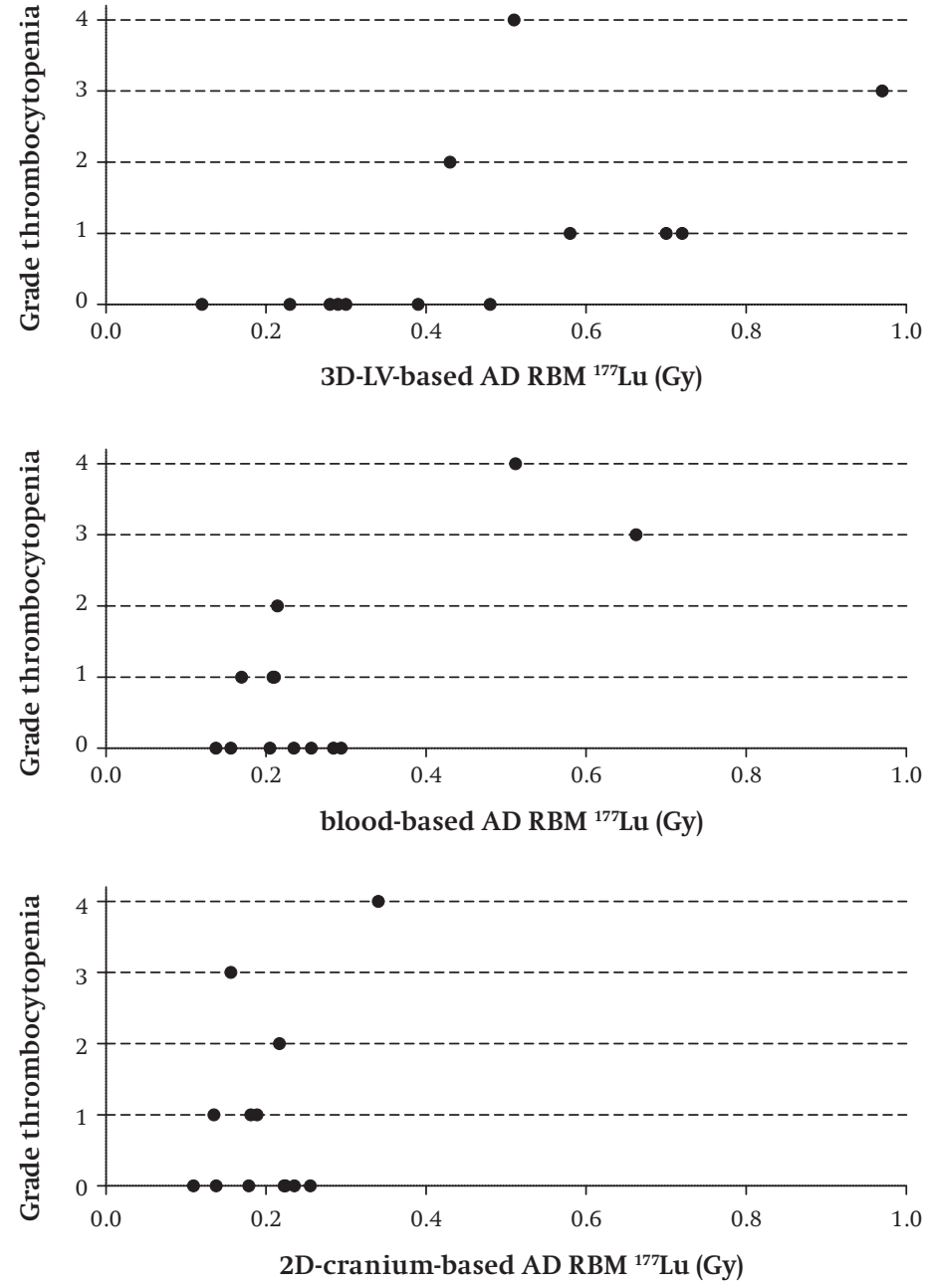

Figure 3 3D-RD-based (A), blood-based (B) and 2D-cranium-based (C) absorbed dose in the red bone marrow, versus the grade of platelet toxicity. AD RBM = absorbed dose red bone marrow.

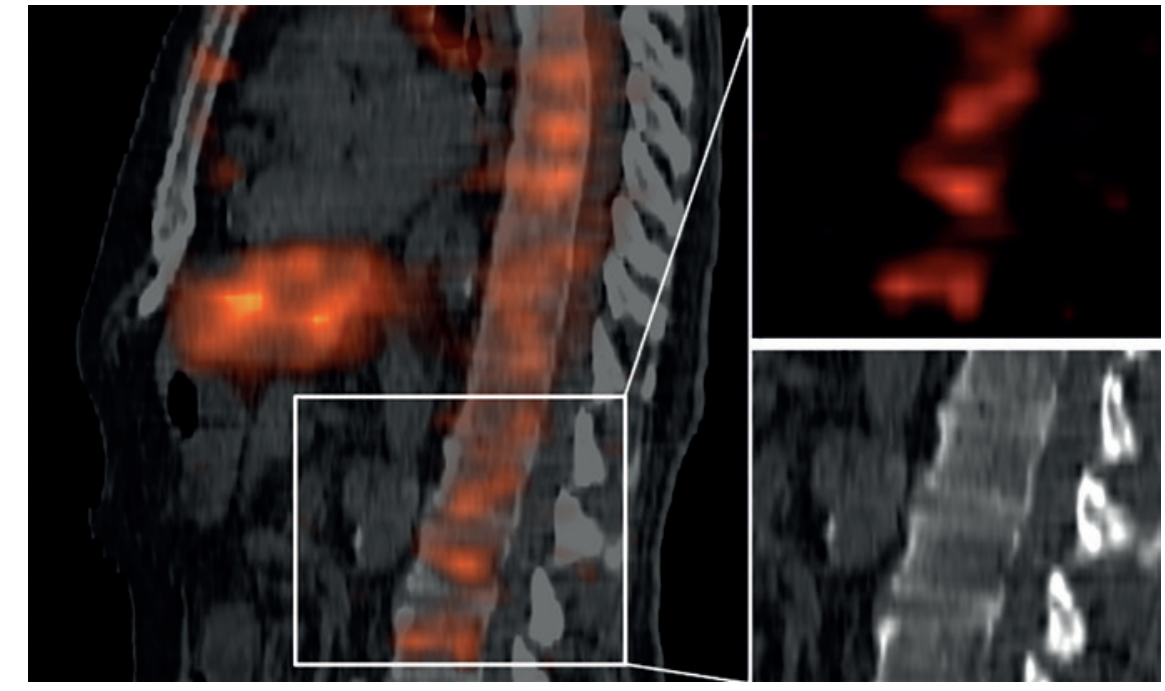

Figure 4 Accumulation of activity in the RBM. Left the saggital cross section of low dose CT and the SPECT image made at 72 hours after administration of the ${ }^{177} \mathrm{Lu}$-peptide of patient 20. Right the SPECT and low dose CT images of vertebrae in which activity accumulated.

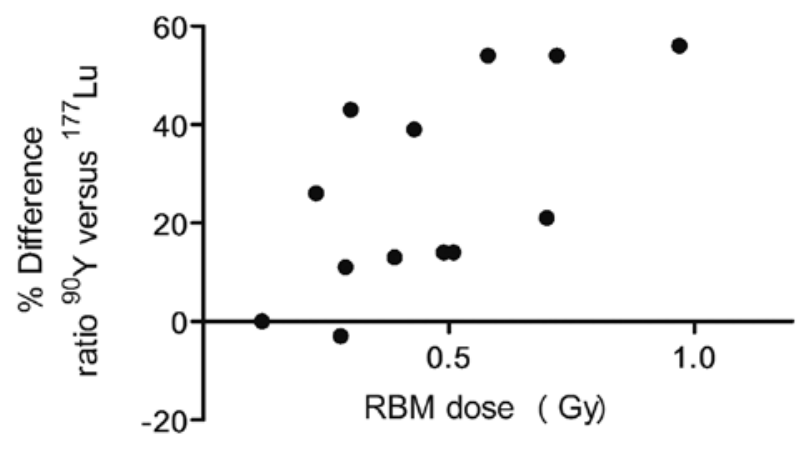

Figure 5 Red bone marrow (RBM) dose versus the percentage difference in ADtumor/ ADRBM for ${ }^{90} \mathrm{Y}$ compared to ${ }^{177} \mathrm{Lu}$. A positive difference represents a higher tumor-to-RBMdose ratio in ${ }^{90} \mathrm{Y}$. 
Table 2 The difference between ADtumor/ADRBM for treatment with ${ }^{177} \mathrm{Lu}$ and ${ }^{90} \mathrm{Y}$. A positive difference represents a higher tumor-to-RBM dose ratio in ${ }^{90} \mathrm{Y}$ and the percentage is calculated compared to the ${ }^{177} \mathrm{Lu}$ ratio.

\begin{tabular}{|l|l|l|l|}
\hline $\begin{array}{l}\text { Patient } \\
\text { number }\end{array}$ & \multicolumn{2}{|l|}{ Ratio tumor/ RBM dose } & \multicolumn{2}{l|}{$\begin{array}{l}\text { Percentage } \\
\text { difference }\end{array}$} \\
\hline $\mathbf{7}$ & 1.87 & ${ }^{90} \mathrm{Y}$ & $13 \%$ \\
\hline $\mathbf{1 0}$ & 5.72 & 2.13 & $14 \%$ \\
\hline $\mathbf{1 1}$ & 8.84 & 6.54 & $11 \%$ \\
\hline $\mathbf{1 2}$ & 5.08 & 9.78 & $43 \%$ \\
\hline $\mathbf{1 3}$ & 4.33 & 7.27 & $0 \%$ \\
\hline $\mathbf{1 4}$ & 5.26 & 4.33 & $21 \%$ \\
\hline $\mathbf{1 5}$ & 1.49 & 2.37 & $54 \%$ \\
\hline $\mathbf{1 6}$ & 0.68 & 1.06 & $56 \%$ \\
\hline $\mathbf{1 7}$ & 1.46 & 2.02 & $39 \%$ \\
\hline $\mathbf{1 8}$ & 9.45 & 8.87 & $-6 \%$ \\
\hline $\mathbf{1 9}$ & 5.11 & 4.97 & $-3 \%$ \\
\hline $\mathbf{2 0}$ & 0.64 & 0.99 & $54 \%$ \\
\hline $\mathbf{2 1}$ & 10.91 & 13.72 & $26 \%$ \\
\hline
\end{tabular}

RBM = red bone marrow

\section{Discussion}

In this study, we demonstrated the utility of 3D-RD for tumor and RBM dose calculation and the correlation with RBM toxicity.

Using 3D-RD, all patients with RBM toxicity had a higher RBM dose than the patients without any RBM toxicity. In contrast, this was not observed with the conventional blood-based and 2D-cranium-based methods which might be caused by an underestimation of the calculated doses.

RBM doses were in most patients higher when calculated with 3D-RD than with the blood-based method (11/13 patients) and the cranium-based method (12/13 patients). In the blood-based RBM dosimetry used, it was assumed that the blood activity concentration is proportional to the RBM concentration at all time points. In most patients, we clearly observed RBM retention at the later imaging time points ( $24 \mathrm{~h}$ and $72 \mathrm{~h}$ post injection). Therefore, at least in these patients, the blood-based method will result in an underestimation for the $\mathrm{AD}_{\mathrm{RBM}}$ and therefore in a poorer correlation with the RBM toxicity, compared to the 3D-RD result.

The $2 \mathrm{D}$ cranium-based dosimetry might also have resulted in an underestimation of the $\mathrm{AD}_{\mathrm{RBM}}$. The fraction of $\mathrm{RBM}$ in the cranium is used to represent the activity in the total RBM. In the SPRIND software package, this fraction was defined as 0.119, based on the ICRP23's Reference Man, representing a 40-year old male. As the median age of our patient-population was 63 years, this fraction might have been an overestimation. Since the distribution of the RBM over the skeleton is both age- and patient-specific, ideally this fraction should be individually measured, and a patient-specific percentage of the RBM in a certain VOI should be used for this method.

For both methods, a patient-specific difference in the severity of the under estimation might explain the relative difference between the methods in relation to the observed RBM toxicity.

In addition, the difference between the 3D LV-based and 2D cranium-based $\mathrm{AD}_{\mathrm{RBM}}$ could be explained by inhomogeneous uptake in the RBM (2) and the influence of activity surrounding the $\mathrm{LV}$ on the $\mathrm{AD}_{\mathrm{H}}$. Taking this into account, the 3D-image-based results seem to be more accurate by avoiding the influence of overlapping organs and due to more accurate VOI drawing because the target-tobackground ratio is higher for 3D than for 2D.

Figure 3A suggests that RBM toxicity can be expected for RBM doses higher than $0.4 \mathrm{~Gy}$. As in all dosimetry studies, the absolute value (i.e., $0.4 \mathrm{~Gy}$ ) is highly influenced by methodological choices such as not to correct for the partial volume effect. This might partially explain the difference with the general rule that a blood (and RBM dose) of $2 \mathrm{~Gy}$ is considered to be safe $(6,27,28)$. Also, and probably most important, all patients had a history of multiple lines of polychemotherapy, which most certainly negatively affected bone marrow reserves and thus myelotoxicity (29).

For actual definition of a threshold, a larger study, including patient groups with and without previous chemotherapy, should be performed.

In this study, attenuation correction was performed using a method that assumes a homogeneous body density, and therefore overestimates the attenuation in low-density tissue like the lungs. To avoid this, CT-based attenuation correction would be preferred for future studies Unfortunately, there was no SPECT-CT available at our department during this clinical study that finished in 2011.

In our view, the absorbed dose -or a derived parameter of the AD (i.e. BED)has the potential to be predictive for different radiolabeled therapeutics, irrespective of the radionuclides, radiolabeled peptides, radiolabeled antibodies or pretargeting. 
As we have shown, 3D-image-based dosimetry seems to result in a clear dose-toxicity relationship and therefore might be a valuable approach for the calculation of a maximally tolerated absorbed dose (MTAD). However, a robust definition of a reliable MTAD based on this method would require processing of larger datasets.

The OLINDA-based results of the tumor-absorbed doses were comparable to the 3D-RD results and are in accordance with previous research (30). In general the OLINDA sphere model led to a somewhat lower dose. This could be explained by the influence of activity surrounding the VOI. For use of the OLINDA sphere model, the $\mathrm{VOI}$ is assumed to be isolated. In 3D-RD, surrounding activity does contribute to the tumor dose, which is more realistic. Nevertheless, we conclude that OLINDA provides a fast and practical method for tumor dosimetry.

OLINDA RBM dosimetry, or more generally S-factor-based RBM dosimetry, is not feasible without a known (or a model-based) RBM mass. Using a dose poin kernel, as alternative for the MC-simulation would reduce the time needed for the calculation. Nevertheless, 3D-RD can easily provide dose rates per time point, and enables delineation for each time point. Therefore, for the calculation of the bone marrow dose we prefer 3D-RD and we consider MC-based software advantageous for RBM dosimetry, despite the fact that it is more time-consuming.

Matched pair dosimetry, as shown for ${ }^{177} \mathrm{Lu}$ and ${ }^{90} \mathrm{Y}$, proved to be a convenient and inexpensive method (compared to animal or patient studies) for exploring the effect of the different radionuclide on the tumor-to-RBM dose ratio. The results of the ${ }^{90} \mathrm{Y}$ simulation confirm the hypothesis that replacing ${ }^{177} \mathrm{Lu}$ by ${ }^{90} \mathrm{Y}$ would lead to an increase in the tumor-to-RBM dose ratio, especially in patients given a higher RBM dose. In such patients, we see a relatively slow, but clear, accumulation of activity in the RBM, which is most visible on the later scans. Therefore, the shorter half-life of ${ }^{90} \mathrm{Y}$ is advantageous.

\section{Conclusions}

3D-RD based RBM dosimetry is feasible and a more sensitive predictor for selection of patients showing any grade of RBM toxicity than blood-based and planar image-based RBM doses. In addition, comparing the ${ }^{177} \mathrm{Lu}$ tumor to RBM dose ratios with the simulated ${ }^{90} \mathrm{Y}$ ratios proved to be a useful method to explore the effect of using a different radionuclide for this therapeutic modality.

\section{Acknowledgements}

This work was supported by the Dutch Cancer Society (KWF Kankerbestrijding, grant no. KUN 2008-4038) and NCI R01CA116477. We thank Antoi P.W. Meeuwis for providing the essential assistance in preparing the data for the dosimetry.

\section{References}

1. Hindorf C, Glatting G, Chiesa C, Linden O, Flux G. EANM Dosimetry Committee guidelines for bone marrow and whole-body dosimetry. European journal of nuclear medicine and molecular imaging. 2010;37(6):1238-50. Epub 2010/04/23.

2. Sgouros G, Jureidini IM, Scott AM, Graham MC, Larson SM, Scheinberg DA. Bone marrow dosimetry regional variability of marrow-localizing antibody Journal of nuclear medicine , 1996:37(4):695-8. Epub 1996/04/01.

3. Shen S, DeNardo GL, Sgouros G, O'Donnell RT, DeNardo SJ. Practical determination of patient-specific marrow dose using radioactivity concentration in blood and body. Journal of nuclear medicine 1999;40(12):2102-6. Epub 2000/01/05.

4 Schwartz J. Humm JL, Divgi CR, Larson SM, O'Donoghue JA. Bone Marrow Dosimetry Using 124I-PET. Journal of nuclear medicine . 2012;53(4):615-21. Epub 2012/03/15.

5. Ferrer L, Kraeber-Bodere F, Bodet-Milin C, Rousseau C Le Goill S, Wegener WA, et al. Three methods

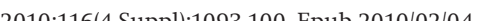

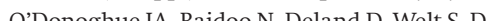

and D, Welt S, Divgi CR, Sgouros G. Hematologic toxicity in radioimmunonships for I-131 labeled antibody therapy. Cancer Biother Radiopharm.

2002;17(4):435-43. Epub 2002/10/25.
7. Siegel JA, Yeldell D, Goldenberg DM, Stabin MG, Sparks RB, Sharkey RM, et al. Red marrow radiatio dose adjustment using plasma FLT3-L cytokine levels: improved correlations between hematologic 作

8. Wessels BW, Bolch WE, Bouchet LG, Breitz HB, Denardo GL, Meredith RF, et al. Bone marrow dosimetr using blood-based models for radiolabeled antibody therapy: a multiinstitutional comparison. Journal of nuclear medicine . 2004;45(10):1725-33. Epub 2004/10/09.

9. Sgouros G, Chiu S, Pentlow KS, Brewster LJ, Kalaigian H, Baldwin B, et al. Three-dimensional dosimetry for hioimmunotherapy treatment planning. Journal of nuclear medicine. 1993;34(9):1595-601. Epub 1993/09/0

D. Brouwers AH, Buijs WC, Mulders PF, de Mulder PH, van den Broek WJ, Mala C, et al. Radioimmunotherapy with [131I] G G250 in patients with metastasized renal cell cancer: dosimetric analysis an immunologic response. Clin Cancer Res. 2005:11(19 Pt 2):7178s-86s. Epub 2005/10/06.

11. Schoffelen R, Woliner-van der Weg W, Visser EP, Goldenberg DM, Sharkey RM, McBride WJ, et al Predictive patient-specific dosimetry and individualized dosing of pretargeted radioimmunotherapy in patients with advanced colorectal cancer. European journal of nuclear medicine and molecula imaging. 2014;41(8):1593-602. Epub 2014/03/20.

12. He B, Wahl RL, Du Y, Sgouros G, Jacene H, Flinn I, et al. Comparison of residence time estimatio methods for radioimmunotherapy dosimetry and treatment planning-Monte Carlo simulatio studies. IEEE Trans Med Imaging. 2008;27(4):521-30. Epub 2008/04/09.

13. Boucek JA, Turner JH. Personalized Dosimetry of I-131-Rituximab Radioimmunotherapy of NonHodgkin Lymphoma Defined by Pharmacokinetics in Bone Marrow and Blood. Cancer Biother Radio. 2014;29(1):18-25.

14. Snyder WS, Ford MR, Warner GG, Watson SB. MIRD Pamphlet \#11: S, Absorbed Dose per Unit Cumulated Activity for Selected Radionuclides and Organs. In: Society of Nuclear Medicine R, VA, editor. 1975 
15. Hobbs RF, Wahl RL, Lodge MA, Javadi MS, Cho SY, Chien DT, et al. 124I PET-based 3D-RD dosimetry for pediatric thyroid cancer patient: real-time treatment planning and methodologic comparison. Journal of nuclear medicine . 2009;50(11):1844-7. Epub 2009/10/20.

16. Prideaux AR, Song H, Hobbs RF, He B, Frey EC, Ladenson PW, et al. Three-dimensional radiobiologic dosimetry: application of radiobiologic modeling to patient-specific 3-dimensional imaging-based internal dosimetry. Journal of nuclear medicine . 2007;48(6):1008-16. Epub 2007/05/17.

17. Boellaard R, O'Doherty MJ, Weber WA, Mottaghy FM, Lonsdale MN, Stroobants SG, et al. FDG PET and PET/CT: EANM procedure guidelines for tumour PET imaging: version 1.0. European journal of nuclear medicine and molecular imaging. 2010;37(1):181-200. Epub 2009/11/17.

18. Visser E. Postema E Boerman O, Visschers J. Oyen W Corstens F. Software package for integrated data processing for internal dose assessment in nuclear medicine (SPRIND). European journal of nucler (1)

Forrer F, Krenning EP, Kooij PP, Bernard BF, Konijnenberg M, Bak

dorit. Bone marrow

. Stabin MG, Sparks RB, Crowe E. OLINDA/EXM: the second-generation personal computer software for internal dose assessment in nuclear medicine. Journal of nuclear medicine . 2005;46(6):1023-7. Epub

21. Brown S, Bailey DL, Willowson K, Baldock C. Investigation of the relationship between linear attenuation coefficients and CT Hounsfield units using radionuclides for SPECT. Appl Radiat Isot. 2008:66(9):1206-12. Epub 2008/07/30.

22. Cherry SR, Sorensen JA, Phelps ME. Physics in Nuclear Medicine. Philadelphia, PA, USA: Saunders, Elsever inc.; 2012

23. Sgouros G, Frey E, Wahl R, He B, Prideaux A, Hobbs R. Three-dimensional imaging-based radiobiological dosimetry. Seminars in nuclear medicine. 2008;38(5):321-34. Epub 2008/07/30.

24. Eckerman KF, Endo A. MIRD: Radionuclide Data and Decay Schemes. 1st ed. Reston, VA: Society of Nuclear Medicine; 2008.

tt T, Baechler S, He B, Esaias CE, Frey EC, et al. A Treatment Planning Method for Sequentially Combining Radiopharmaceutical Therapy and External Radiation Therapy. Int J Radiat Oncol Biol Phys. 2010. Epub 2010/10/19

26. Baechler S, Hobbs RF, Prideaux AR, Wahl RL, Sgouros G. Extension of the biological effective dose to the MIRD schema and possible implications in radionuclide therapy dosimetry. Medical physics. 2008,35(3).1123-34. Еpub 2008/04/15.

27. Benua RS, Leeper, R.D. A method and rationale for treating metastatic thyroid carcinoma with the largest safe dose of I-131. In: Medeiros-Neto G, Gaitan, G., editor. Frontiers in thyroidology. New Yor Plenum Medical Book Co; 1986. p. 1317-21.

28. Benua RS, Cicale NR, Sonenberg M, Rawson RW. The relation of radioiodine dosimetry to results and complications in the treatment of metastatic thyroid cancer. Am J Roentgenol Radium Ther Nucl Med 1962;87:171-82. Epub 1962/01/01

29. Baechler S, Hobbs RF, Jacene HA, Bochud FO, Wahl RL, Sgouros G. Predicting hematologic toxicity in patients undergoing radioimmunotherapy with 90Y-britumomab tiuxetan or 131I-tositumomab. Journal of nuclear medicine . 2010;51(12):1878-84. Epub 2010/11/26.

30. Senthamizhchelvan S, Hobbs RF, Song H, Frey EC, Zhang Z, Armour E, et al. Tumor dosimetry and response for $153 \mathrm{Sm}$-ethylenediamine tetramethylene phosphonic acid therapy of high-ris osteosarcoma. Journal of nuclear medicine . 2012;53(2):215-24. Epub 2012/01/19, 
4

Non-invasive quantification of the beta cell mass by SPECT with

${ }^{111}$ In-exendin

Maarten Brom*, Wietske Woliner - van der Weg*, Lieke Joosten, Cathelijne Frielink,

Thomas Bouckenooghe, Paul Rijken, Karolina Andralojc, Burkhard J. Göke, Marion de Jong, Decio L. Eizirik, Martin Béhé, Tony Lahoutte, Wim J.G. Oyen, Cees J. Tack, Marcel Janssen,

Otto C. Boerman, Martin Gotthardt

"These authors contributed equally 


\section{Abstract}

A reliable method for in vivo quantification of pancreatic beta cell mass (BCM) could lead to further insight in the pathophysiology of diabetes. The glucagon-like peptide 1 receptor (GLP-1R), abundantly expressed on beta cells, may be a suitable target for imaging. We investigated the potential of radiotracer imaging with the GLP-1 analogue ${ }^{111}$ In-exendin for determination of BCM in vivo in a rodent model of beta cell loss and in type 1 diabetes (T1D) patients and healthy volunteers.

Methods: The targeting of ${ }^{111}$ In-exendin was examined in a rat model for alloxaninduced beta cell loss. Rats were injected with $15 \mathrm{MBq}{ }^{111}$ In-exendin and Single Photon Emission Computed Tomography (SPECT) acquisition was performed $1 \mathrm{~h}$ p.i., followed by dissection, biodistribution and ex vivo autoradiography studies of pancreatic sections. BCM was determined by morphometric analysis after staining with an anti-insulin antibody. For clinical evaluation SPECT was acquired 4, 24 and $48 \mathrm{~h}$ after injection of $150 \mathrm{MBq}{ }^{111} \mathrm{In}$-exendin in 5 T1D patients and 5 healthy volunteers. The tracer uptake was determined by quantitative analysis of the SPECT images.

Results: In rats, ${ }^{111}$ In-exendin specifically targets the beta cells and pancreatic uptake is highly correlated with BCM. In patients, the pancreas was visible in SPECT images and the pancreatic uptake showed high interindividual variation with a substantially lower uptake in T1D patients.

Conclusions/interpretation: These studies indicate that ${ }^{111}$ In-exendin may be suitable for non-invasive quantification of BCM.

Trial registry number: NCT01825148 on www.clincaltrials.gov, EudraCT: 2012-000619-10

\section{Introduction}

Diabetes mellitus is characterized by chronically elevated blood glucose levels. In type 1 diabetes (T1D), autoimmune destruction of pancreatic beta cells leads to absolute deficiency of insulin secretion with subsequent hyperglycaemia (1). It is commonly assumed that T1D becomes clinically apparent when remaining beta cell mass (BCM) has decreased to $5-20 \%(2,3)$. Recent studies, however, suggest that T1D patients may still have a large amount of remaining beta cells (4) and in some patients, loss of BCM was less than 50\% (5). Moreover, some newly diagnosed T1D patients who underwent autologous nonmyeloablative hemotopoietic stem cell transplantation became insulin-independent for several months, suggesting restored function of existing beta cells (6). In type 2 diabetes (T2D), failure of the beta cells to adapt to the increased insulin demand, posed by insulin resistance in target tissues, leads to impaired glucose homeostasis $(1,7-10)$. BCM changes during the development and course of $\mathrm{T} 2 \mathrm{D}$ (11), but this process is neither well understood nor well characterized (9). Moreover, it also appears that BCM and beta cell function do not always correlate over the course of disease $(9,12)$. To date, no reliable method exists to measure BCM in humans in vivo and most information about human BCM has been obtained from autopsy studies of diabetic patients (9) and healthy subjects. Autopsies, however, cannot show the dynamics of BCM at onset and progression of the disease. Therefore, a non-invasive imaging method to quantify BCM in vivo would allow us to gain better insight into the pathophysiology of diabetes during progression of the disease.

Magnetic resonance imaging (MRI) and computed tomography (CT) offer the highest resolution of currently available clinical imaging technology, but neither resolution nor contrast suffices to visualize single pancreatic islets in humans in vivo. As an alternative, nuclear medicine imaging modalities such as SPECT (Single Photon Emission Computed Tomography) or PET (Positron Emission Tomography) offer a very high sensitivity which may be sufficient for the detection of the relatively low number of pancreatic islet cells by using a highly beta cell-specific radiolabelled tracer molecule. A potential target for measurement of the BCM is the glucagon-like peptide 1 receptor (GLP-1R) as it is abundantly expressed in rat, mouse and human pancreatic beta cells but not in , and pp cells $(13,14)$. Exendin is a stable agonist of the GLP-1R and it was previously used for in vivo targeting of the GLP-1R after labelling with various radionuclides (15-19). Exendin labelled with ${ }^{111}$ In could be used to determine the BCM in vivo by SPECT.

To test this hypothesis, we first explored biodistribution and specificity of the ${ }^{111}$ In-exendin tracer in a rat model of alloxan-induced beta cell loss and subsequently in 5 T1D patients and 5 healthy controls. 


\section{Materials and Methods}

\section{Peptides and radiolabelling for studies in rats}

$\left[\right.$ Lys $\left.^{40}(\mathrm{DTPA})\right]$ exendin-3 and $\left[\right.$ Lys $\left.^{40}\right]$ exendin-3 were purchased from Peptides Specialty Laboratories (Heidelberg, Germany). DTPA was conjugated to the -amino group of the Lysine (K40) and the C-terminal carboxyl group was amidated (15). ${ }^{111}$ In-labelling and quality control was performed as previously described (15). ${ }^{111}$ In-exendin was injected in rats without further purification.

\section{SPECT and biodistribution of diabetic rats}

In order to determine the correlation of the uptake of ${ }^{111}$ In-exendin-3 and the beta cell mass, six to eight week old female Brown Norway rats (Harlan, Horst, The Netherlands) were injected with various concentrations of alloxan monohydrate (Sigma Chemicals, St. Louis, MO, USA): 15, 30, 45 or $60 \mathrm{mg} / \mathrm{kg}$ body weight ( $\mathrm{n}=4$ per group). Alloxan was dissolved in ice-cold $10 \mathrm{mM} \mathrm{HCl}$ at a concentration of $25 \mathrm{mg}$ $\mathrm{ml}$, diluted with ice-cold PBS and protected from light during storage and dilution. Rats were injected in the tail vein with $200 \mu \mathrm{l}$ alloxan solution within 5 minutes after dilution in $10 \mathrm{mM} \mathrm{HCl}$, while the control group $(\mathrm{n}=4)$ was injected with $\mathrm{HCl}$ in PBS. Blood glucose concentrations were determined one week after injection of alloxan using a glucose meter (Accu-Chek Sensor, Roche Diagnostics, Almere, The Netherlands). One week after the injection of alloxan, when hyperglycaemia was confirmed, rats received $15 \mathrm{MBq}{ }^{111}$ In-exendin-3 intravenously; 5 control rats were co-injected with an excess of unlabelled exendin-3 $(25 \mu \mathrm{g})$. One hour p.i., SPECT/CT scans were acquired using a dedicated small animal SPECT/CT scanner (NanoSPECT) CT, Bioscan, Paris, France). SPECT images were acquired with a $2.5 \mathrm{~mm}$ multipinhole rat collimator, 24 projections of 120 sec were acquired. SPECT images were reconstructed with HiSPECT software (noise suppression high, standard voxel size standard $(0.3 \mathrm{~mm})$, standard reconstruction settings). After SPECT imaging, the pancreas and other relevant tissues were dissected, weighed and counted in a well type -counter (Wallac 1480-Wizard, Perkin-Elmer, Boston, MA, USA). Pancreata of one rat per group were harvested and snap-frozen in isopentane on dry ice for autoradiography. Cryosections $(10 \mu \mathrm{m})$ were exposed to an imaging plate (Fuji Film BAS-SR 2025, Raytest, Straubenhardt, Germany) for $72 \mathrm{~h}$. Images were acquired with a radioluminography laser imager (Fuji Film BAS 1800 II system, Raytest, Straubenhardt, Germany) and analyzed with Aida Image Analyzer software (Raytest). The pancreata of the remaining rats were fixed in formalin and embedded in paraffin for the determination of the beta cell mass as described below.

Pancreatic BCM was determined as described below and plotted against the pancreatic uptake determined by ex vivo counting; the correlation was determined by the Pearson correlation coefficient (r) using GraphPad Prism (version 4).
${ }^{111}$ In-exendin-3 uptake was quantified using Inveon Research Workplace (Preclinical Solutions, Siemens Medical Solutions USA, Inc., Knoxville, TN, USA) by drawing a volume of interest $(8 \mu \mathrm{l})$ over the pancreatic region and correcting it for the injected activity and non-specific uptake (determined by co-injection of an excess unlabelled exendin-3).

\section{SPECT of dissected tissues}

Brown Norways rats were injected intravenously with $60 \mathrm{mg} / \mathrm{kg}$ alloxan monohydrate (Sigma Chemicals, St. Louis, MO, USA) as described above. When hyperglycaemia was confirmed, diabetic $(n=4)$ and healthy $(n=4)$ rats were injected with $15 \mathrm{MBq}{ }^{111} \mathrm{In}$-exendin-3. Two control rats were co-injected with an excess of unlabelled exendin-3 $(25 \mu \mathrm{g} / \mathrm{rat})$. Rats were euthanized $1 \mathrm{~h}$ after injection of the radiolabeled exendin and the pancreas, gastro intestinal tract, liver and spleen were dissected and fixed in $4 \%$ formalin. SPECT images of the dissected pancreata along with the gastro-intestinal tract, liver and spleen were acquired on a dedicated small animal SPECT scanner (U-SPECT-II, MILabs, Utrecht, The Netherlands). SPECT images were acquired with a $1.0 \mathrm{~mm}$ pinhole rat collimator with an acquisition time of $4 \mathrm{~h}$. The images were reconstructed with OSEM (6 iterations, 16 subsets, voxel size 0.1875 ) using the U-SPECT-Rec software (MILabs, Utrecht, The Netherlands).

\section{Quantitative analysis of SPECT images of rats.}

${ }^{111}$ In-exendin-3 uptake was quantified using Inveon Research Workplace (Preclinica Solutions, Siemens Medical Solutions USA, Inc., Knoxville, TN, USA) by drawing a volume of interest $(8 \mu \mathrm{l})$ over the pancreatic region and correcting it for the injected activity and non-specific uptake (determined by co-injection of an excess unlabeled exendin-3). The volume of interest was placed in the coronal plane, cranial/anterio of the kidneys in the same plane where the high uptake was observed in the transition of the stomach to the duodenum (see Figure 1). The absolute activity (in Bq) was calculated by multiplying the value with the calibration factor determined by quantitative analysis of standards, scanned with the same acquisition settings.

To determine the exact localization of the pancreas in the SPECT images, a Brown Norway rat was co-injected with ${ }^{99 \mathrm{~m}} \mathrm{Tc} \mid \mathrm{Demobesin} 3$, a peptide specifically targeting the gastrin-releasing peptide receptor in the exocrine pancreas. ${ }^{99 \mathrm{~m}} \mathrm{Tc}$ Demobesin 3 was labeled with ${ }^{99 m} \mathrm{Tc}$ as previously described (20). Rats were injected intravenously with $20 \mathrm{MBq}$ (approximately $2 \mu \mathrm{g}$ ) diluted in PBS containing 0.5\% BSA (v/w) and 15 MBq ${ }^{11}$ In-exendin-3. SPECT was acquired for ${ }^{99 m} \mathrm{Tc}$ and ${ }^{111} \mathrm{In}$ separately, 60 min p.i. (NanoSPECT/CT, Bioscan, Paris, France). After SPECT acquisition, the rat was euthanized and the kidneys were removed in order to avoid overprojection on projection images followed by an additional SPECT acquisition. Images were fused and analyzed using Inveon Research Workplace (Siemens Inc, Munich, Germany). 


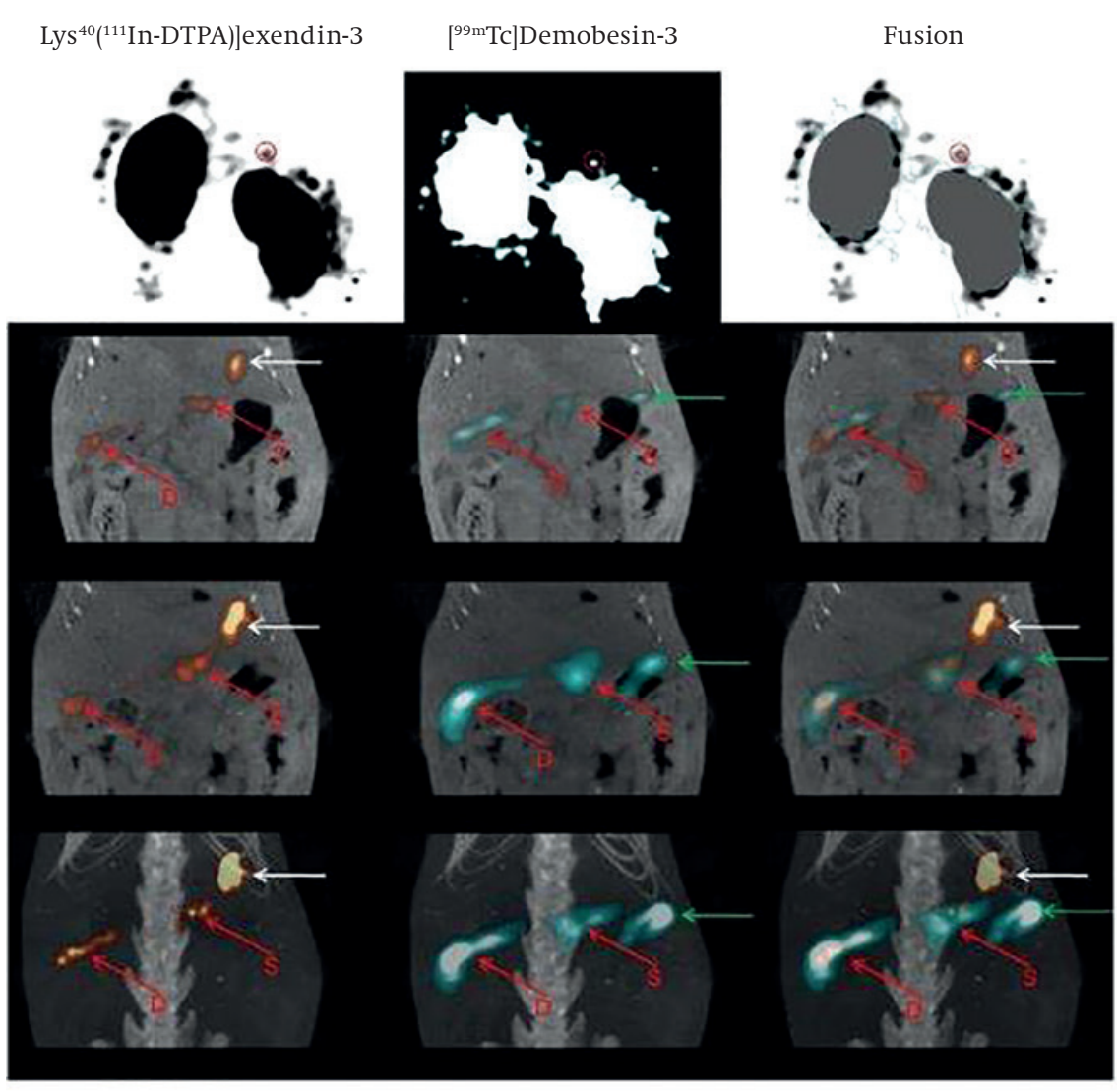

Figure 1 SPECT images of a Brown Norway rat after injection of [99m Tc]Demobesin-3 and ${ }^{111} \mathrm{In}$-exendin (A). Pancreatic tissue on $\left[{ }^{99 \mathrm{~m}} \mathrm{Tc}\right]$ Demobesin scan colocalized with the region observed in the ${ }^{111}$ In-exendin scans (Pancreata are indicated with red circles). SPECT images show clear colocalization of $\left.{ }^{99 m} \mathrm{mc}\right]$ Demobesin and ${ }^{111} \mathrm{In}$-exendin in the pancreatic region (B-C: coronal slices, D: Maximum intensity projection (MIP), red arrow: pancreas (S: splenic part, D: duodenal part), white arrow: stomach, green arrow: spleen; kidneys removed (for these images only) in order to avoid over-projection on MIP).

\section{Determination of the beta cell mass}

Five sections $(4 \mu \mathrm{m})$ of paraffin-embedded pancreata, $100 \mu \mathrm{m}$ apart from each other were stained with an anti-insulin antibody $(4 \mu \mathrm{g} / \mathrm{ml}$ in PBS containing $1 \%$ BSA w/v) (sc 9168, Santa Cruz Biotechnology, Inc., Santa Cruz, CA, USA.) as previously described (21).
The surface of the beta cells in the sections was estimated by drawing regions of interest around insulin stained tissue and total pancreatic tissue on images of the pancreatic section that were captured using a digital image processing system for bright field microscopy (22) (400x magnification). The total pancreatic pixel area was determined by automated image thresholding (23) of the haematoxylin stained pancreatic tissue. The total insulin-positive pixel area was determined by drawing regions of interest over the insulin-positive tissue and the insulin-positive fraction was calculated by dividing the insulin positive pixel area by the total pancreatic pixel area (24). The beta cell mass was calculated by multiplying the insulin positive fraction with the total pancreatic weight.

\section{Microautoradioraphy}

One Brown Norway rat was injected intravenously 3 times with $15 \mathrm{MBq}{ }^{111} \mathrm{In}-$ exendin- 3 with an interval of $1 \mathrm{~h}$ and the pancreas was harvested $1 \mathrm{~h}$ after the last injection. The pancreas was fixed in formalin and embedded in paraffin and $4 \mu \mathrm{m}$ sections were prepared. The sections were deparaffinised and covered with a homogeneous layer of hypercoat emulsion (Amersham LM-1, GE Healthcare UK limited, Buckinghamshire, UK) by dipping the slides in the emulsion at $42 \mathrm{C}$ in the dark. After incubation in the dark for one week the sections were developed for $4 \mathrm{~min}$ in a $6 \%$ black and white developer (Agfa studional liquid, Agfa, Leverkusen, Germany). The sections were rinsed in water for $20 \mathrm{sec}$ and fixed in $24 \%(\mathrm{w} / \mathrm{v})$ solution of sodium thiosulphate for $4 \mathrm{~min}$. The sections were rinsed with water stained with heamatoxylin for $30 \mathrm{sec}$, rinsed with water for $10 \mathrm{~min}$, dehydrated and the slide was mounted with mounting fluid. Consecutive sections were stained with an anti-insulin monoclonal antibody as described above for co-localization.

\section{mRNA extraction and real time PCR}

Poly(A)+ mRNA were isolated from rat tissues using the Dynabeads mRNA DIRECT ${ }^{\text {TM }}$ kit (Invitrogen, Merelbeke, Belgium), and reverse transcribed as previously described (25). Human cDNA were from Gentaur (Brussels, Belgium). The real time PCR amplification reaction was done as described (26), using iQ SyBR Green Supermix on iCycler MyiQ Single Color (BIO-RAD, Hercules, CA, USA) and compared to a standard curve (27). Expression values were corrected for the housekeeping gene glyceraldehyde-3-phosphate dehydrogenase (GAPDH). Primers used are summarized in Table 1.

\section{Preparation and labelling of ${ }^{111} \mathrm{In}$-exendin for human use}

$\left[\operatorname{Lys}^{40}(A h x-D T P A)\right]$ exendin-4 for clinical use was produced (piCHEM Forschungs-und Entwicklungs-GmbH, Graz, Austria), dissolved and aliquoted according to GMP standards to a final concentration of $2 \mu \mathrm{g} / \mathrm{ml}$ in 1M HEPES (pH5.5) containing $0.1 \%$ 
Table 1 Primers used for quantitative PCR

\begin{tabular}{l|l|l|l|}
\hline \multirow{2}{*}{ Rat GAPDH } & & Sequence $\mathbf{( 5}^{\prime}$ - $\mathbf{3}^{\prime}$ ) & Base-pairs \\
\hline \multirow{2}{*}{ Rat GLP-1R } & AGTTCAACGGCACAGTCAAG & 118 \\
& R & TACTCAGCACCAGCATCACC & \\
\hline \multirow{2}{*}{ Human GAPDH } & F & GCTGCCCTCAAGTGGATGTA & 108 \\
& R & ATGAGCAGGAACACCAGTCG & \\
\hline \multirow{2}{*}{ Human GLP-1R } & AGTTCAACGGCACAGTCAAG & 136 \\
\cline { 2 - 4 } & R & TACTCAGCACCAGCATCACC & \\
\hline & F & TCCTCCTCGGCTTCAGACACCTGCA & 114 \\
& R & CCACTTCAGGGCTGCGTCCTTGATG & \\
\hline
\end{tabular}

Tween 80 . The vials were stored at $-20^{\circ} \mathrm{C}$. Radiolabelling was performed by adding exendin to the vial containing ${ }^{111} \mathrm{InCl}_{3}$ to a final concentration of $150 \mathrm{MBq}{ }^{111} \mathrm{InCl}_{3}$ per $\mu \mathrm{g}\left[\operatorname{Lys}^{40}(\mathrm{Ahx}\right.$-DTPA) $]$ exendin-4. After incubation at room temperature for 30 min $0.25 \mathrm{ml}$ EDTA $(3.75 \mathrm{mg} / \mathrm{ml})$ was added and quality control was performed as described above. Finally, the volume was adjusted to $5 \mathrm{ml}$ with $0.9 \% \mathrm{NaCl}$ before administration.

\section{Type 1 diabetes patients and healthy controls}

Five patients with type 1 diabetes (T1D), who met the inclusion criteria listed in Table 2, were included in this study. Only T1D patients without insulin production as confirmed by undetectable non-stimulated and stimulated C-peptide $\quad<0.03$ nmol/1), were included. Non-stimulated C-peptide levels were measured after fasting for at least 4 hours. Six minutes after stimulation of the insulin production with $1 \mathrm{mg}$ glucagon a blood sample for measuring the stimulated C-peptide was taken. In order to create two comparable groups, healthy controls matched for BMI (maximum difference $2 \mathrm{~kg} / \mathrm{m}^{2}$ ), age (maximum difference 10 year) and sex were selected for participation. Only healthy controls with $\mathrm{HbA}_{1 \mathrm{C}}<7 \%$ and normal glucose tolerance, assessed by an oral glucose tolerance test (OGTT), were included. After fasting for at least 10 hours, subjects drank 75 gram of anhydrous glucose dissolved in $250 \mathrm{ml}$ water within 5 minutes. The blood glucose $2 \mathrm{~h}$ after glucose intake, had to be below $7.8 \mathrm{mmol} / 1$ (28).

An overview of the subjects included in the study is given in Table 3. From all subjects written informed consent in accordance with provisions of the Declaration of Helsinki was obtained, and the study was approved by the Institutional Ethics Review Board of the Radboud University Nijmegen Medical Centre.

\begin{tabular}{l|l|}
$\begin{array}{l}\text { Table } 2 \text { Inclusion criteria } \\
\text { BMI }\end{array}$ & range \\
\hline Age & $18.5-27 \mathrm{~kg} / \mathrm{m}^{2}$ \\
\hline $\begin{array}{l}\text { No previous treatment with Exenatide or } \\
\text { Dipeptidyl-Peptidase IV inhibitors }\end{array}$ & $21-60 \mathrm{y}$ \\
\hline Creatinine clearance (MDRD) & \\
\hline For T1D patients: & $\geq 40 \mathrm{~mL} / \mathrm{min}$ \\
\hline No stimulated insulin production:C-peptide & \\
\hline Time since diagnosis T1D & $\begin{array}{l}\text { Not measurable } \\
(<0.03 \mathrm{nmol} / \mathrm{L})\end{array}$ \\
\hline $\begin{array}{l}\text { For healthy controls: } \\
\text { HbA } 1 \mathrm{C}\end{array}$ & $>5 \mathrm{y}$ \\
\hline Normal glucose tolerance: Blood glucose $2 \mathrm{~h}$ after OGTT & $<7.8 \mathrm{mmol} / \mathrm{L}$ \\
\hline OGTT = oral glucose tolerance test & \\
\hline
\end{tabular}

\section{Administration of radiotrace}

After at least 4 hours of fasting, $150 \mathrm{MBq}{ }^{111}$ In-exendin, corresponding to a peptide dose of $1 \mu \mathrm{g}$ exendin, was injected as a slow bolus over 5 minutes.

\section{Image acquisition}

SPECT was acquired $4 \mathrm{~h}, 24 \mathrm{~h}$ and $48 \mathrm{~h}$ after ${ }^{111}$ In-exendin administration using an integrated SPECT/CT scanner (Symbia T16, Siemens Healthcare, Molecular Imaging, Hoffman Estates, IL, USA) equipped with parallel-hole medium-energy collimators. All subjects were scanned with a fixed radius of rotation $(25 \mathrm{~cm})$ and a fixed bed height $(13 \mathrm{~cm}) .128$ views were obtained in step-and-shoot mode (64 views per camera head) with an acquisition time of 40s per view.

Symmetric 15\% windows over the 172 and the $247 \mathrm{keV}$ photopeaks were used, with additional 7\% lower and upper scatter windows.

CT images were acquired $24 \mathrm{~h}$ post-injection with the following settings: 110 $\mathrm{keV}, 45 \mathrm{mAs}$ (using Siemens' "care dose" technology for automatic dose reduction) $39 \mathrm{~cm}$ scanning length, with $5 \mathrm{~mm}$ slices and a pitch of 1 . 
Table 3 Overview included subjects

\begin{tabular}{|c|c|c|c|c|c|c|c|}
\hline & $\begin{array}{l}\text { Age } \\
\text { (years) }\end{array}$ & $\begin{array}{l}\text { BMI } \\
\left(\mathrm{kg} / \mathrm{m}^{2}\right)\end{array}$ & Sex & $\begin{array}{l}\text { T1D } \\
\text { since }\end{array}$ & $\begin{array}{l}\mathrm{HbA}_{1 \mathrm{C}} \\
(\%)\end{array}$ & $\begin{array}{l}\text { CT-based } \\
\text { pancreas } \\
\text { volume } \\
\left(\mathrm{cm}^{3}\right)\end{array}$ & $\begin{array}{l}\text { Uptake } \\
\text { in the whole } \\
\text { pancreas } \\
\text { (counts) }\end{array}$ \\
\hline \multicolumn{8}{|c|}{$\begin{array}{l}\text { Subjects } \\
\text { with T1D: }\end{array}$} \\
\hline D1 & 50 & 26.1 & M & $30 y$ & 7.3 & 51.6 & 239 \\
\hline D2 & 25 & 21.3 & F & $13 \mathrm{y}$ & 8.4 & 63.6 & 1445 \\
\hline D3 & 47 & 23.6 & M & $27 \mathrm{y}$ & 6.7 & 45.9 & 185 \\
\hline D4 & 54 & 21.9 & $\mathrm{~F}$ & $33 \mathrm{y}$ & 7.4 & 20.7 & 92 \\
\hline D5 & 41 & 25.2 & $\mathrm{~F}$ & $27 \mathrm{y}$ & 6.7 & 72.1 & 612 \\
\hline \multicolumn{8}{|c|}{$\begin{array}{l}\text { Healthy } \\
\text { controls: }\end{array}$} \\
\hline H1 & 54 & 24.3 & M & & 5.6 & 88.8 & 263 \\
\hline $\mathrm{H} 2$ & 24 & 19.8 & $\mathrm{~F}$ & & 5.6 & 72.7 & 1821 \\
\hline H3 & 40 & 23.9 & M & & 5.3 & 106.6 & 1844 \\
\hline H4 & 54 & 22.4 & $\mathrm{~F}$ & & 5.4 & 73.8 & 899 \\
\hline H5 & 43 & 24.1 & $\mathrm{~F}$ & & 5.4 & 83.1 & 1577 \\
\hline
\end{tabular}

\section{SPECT reconstruction}

SPECT images were reconstructed with Hermes Hybrid Recon software (Hermes Medical Solutions, Stockholm, Sweden), in 6 iterations, 16 subsets and a matrix size of $128 \times 128$ (corresponding with an isotropic voxel size of $4.7952 \mathrm{~mm}$ ). CT-based attenuation correction and Monte Carlo based scatter correction was performed. Post-reconstruction filtering was applied with a $1.0 \mathrm{~cm}$ Gauss filter.

\section{Quantification of SPECT images}

Image quantification was performed with Inveon Research Workplace software (Siemens Inc, Munich, Germany). Within the pancreas two spherical volumes of interest (VOIs), one in the head and one in the corpus, were used for determination of the pancreatic uptake.

A threshold-based VOI around the kidneys was drawn with a cut-off value of 200 counts and increased by a rim of 3 voxels in width; this area was not taken into account for quantification of uptake even if pancreatic tissue was lying within this VOI in order to avoid spill-over effects from the kidneys. The uptake in the spherical VOIs in the pancreas (as mean counts per voxel) was corrected for the total pancreas volume based on delineation on CT images.
The uptake of the whole pancreas (U, in counts/ pancreas) was calculated with the counts $(C)$ per $\mathrm{mm}^{3}$ within the SPECT VOIs, multiplied by the volume of the whole pancreas $\left(V\right.$, in $\left.\mathrm{mm}^{3}\right)$ as determined on $\mathrm{CT}$ and corrected for administered activity (AA, in MBq), the time after injection $\left(\mathrm{T}_{\text {scan }}-T_{\text {injection }}\right)$ and the half life of ${ }^{111} \operatorname{In}\left(\mathrm{T}_{1 / 2}, 2.81 \mathrm{~d}\right)$ :

$$
U=\frac{C \times V}{A A \times\left(\frac{1}{2}\right)^{\left(T_{\text {sann }}-T_{\text {injection }}\right) / T_{1 / 2}}}
$$

\section{Statistical analysis}

All mean values are expressed as mean \pm standard deviation (SD). Statistica analysis was performed using unpaired two-tailed unpaired t-test using GraphPad Prism (version 4). The level of significance was set at $\mathrm{p}<0.05$.

Correlation between the BCM and pancreatic uptake was determined by the Pearson correlation coefficient (r) using two-tailed analysis of variance with GraphPad Prism (version 4). The level of significance was set at $\mathrm{p}<0.05$.

\section{Results}

\section{Radiolabelling}

A specific activity of $700 \mathrm{GBq} / \mu \mathrm{mol}$ for ${ }^{111}$ In-exendin-3 was obtained with a radiochemical purity exceeding $95 \%$.

\section{Biodistribution studies}

Biodistribution in Brown Norway rats $1 \mathrm{~h}$ p.i. showed tracer uptake in various organs (Fig. 2A). ${ }^{111}$ In-exendin accumulated in the pancreas to a concentration of $0.29 \pm 0.04 \% \mathrm{ID} / \mathrm{g}$. Coinjection of an excess unlabelled exendin resulted in a marked decrease in pancreatic uptake $(0.04 \pm 0.01 \% \mathrm{ID} / \mathrm{g})$, indicating that the uptake of the tracer is GLP-1R mediated, which also holds for the uptake in the stomach and the duodenum $(0.33 \pm 0.03 \% \mathrm{ID} / \mathrm{g}$ and $0.44 \pm 0.08 \% \mathrm{ID} / \mathrm{g}$, respectively). In addition, very high specific uptake was observed in the lungs, known to express high levels of GLP-1R in rodents: $13.45 \pm 2.50 \% \mathrm{ID} / \mathrm{g}$. Kidney uptake was also very high $(29.2 \pm 2.3$ $\% \mathrm{ID} / \mathrm{g}$ ) and could not be blocked with an excess of unlabelled exendin. Severely diabetic rats showed an $80 \%$ reduction of pancreatic uptake $(0.06 \pm 0.01 \% \mathrm{ID} / \mathrm{g})$.

The uptake of ${ }^{111}$ In-exendin in the pancreas was plotted against the BCM (Fig. 2F) showing a good correlation between these two parameters (Pearson $\mathrm{r}=0.89$ ). 


\section{Ex vivo autoradiography}

Autoradiography of pancreatic sections of healthy and alloxan-treated rats demonstrated that the tracer specifically accumulated in the islets of Langerhans: High activity concentrations of ${ }^{11}$ In-exendin were observed in hot spots throughout the pancreatic section in control rats representing the islets of Langerhans (Fig. 2B-C). The uptake was GLP-1R mediated since it could be blocked by an excess of unlabelled exendin (Fig. 2B). Very low uptake of the tracer in the exocrine pancreas was observed (Fig. 2B-C). When treated with alloxan, tracer uptake in the islets was reduced (Fig. 2B).

A

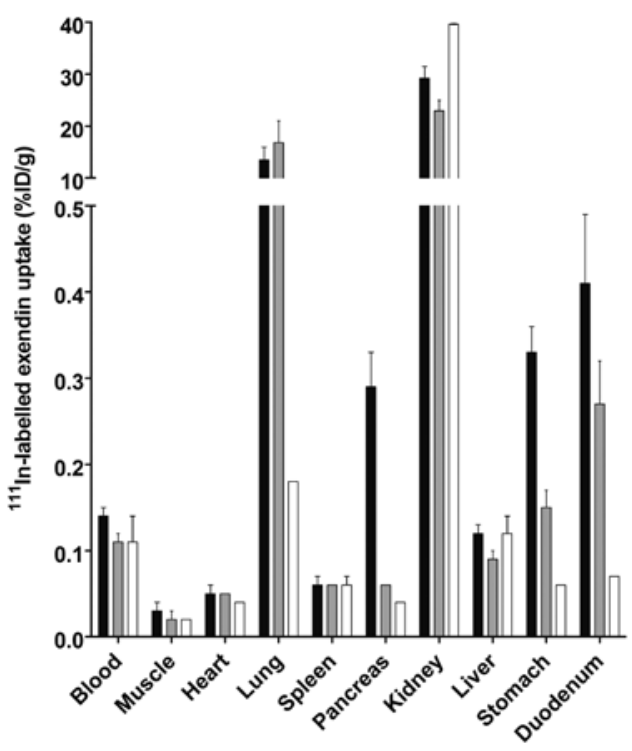

Figure 2 A. Biodistribution of ${ }^{111}$ In-exendin in control $(\mathrm{n}=4)$ and alloxan-treated $(60 \mathrm{mg} / \mathrm{kg}$ (n=4) Brown Norway rats in \% injected dose/g, blocking by co-injection of a 250-fold mola excess of unlabelled exendin $(\mathrm{n}=2)$. Organs were dissected $1 \mathrm{~h}$ post-injection (p.i.). B. Macroautoradiography of consecutive pancreatic sections $(10 \mu \mathrm{m})$ of control and alloxan $(15-60 \mathrm{mg}$ $\mathrm{kg}$ ) treated Brown Norway rats $\left(15 \mathrm{MBq}{ }^{11} \mathrm{In}\right.$-exendin $(0.1 \mu \mathrm{g})$, dissection $1 \mathrm{~h}$ p.i.) C. A section $(4 \mu \mathrm{m})$ of pancreatic tissue (embedded in paraffin) of a control rat (magnification $4 \mathrm{x}$ ) was counterstained with an anti-insulin $\mathrm{mAb}$ (brown) after exposure to the imaging plate. The immunohistochemically stained islets are connected with the representative radiosignal with arrows on enlarged section. D. Microautoradiography shows exclusive uptake in the islets and excellent correlation with immunohistochemical staining (E) with anti-insulin $\mathrm{mAb}$. F. Correlation between the uptake of ${ }^{111}$ In-exendin in rats injected with alloxan $(0,15$, 30,45 and $60 \mathrm{mg} / \mathrm{kg}$ ) and BCM. The uptake on the $\mathrm{x}$-axis is expressed as \% $\mathrm{ID} / \mathrm{g}$ and BCM (in $\mathrm{mg}$ ) as determined by morphometric analysis after immunohistochemical staining with an anti-insulin antibody on the $\mathrm{y}$-axis. The correlation as determined by Pearson test is $\mathrm{r}=0.89$.
B

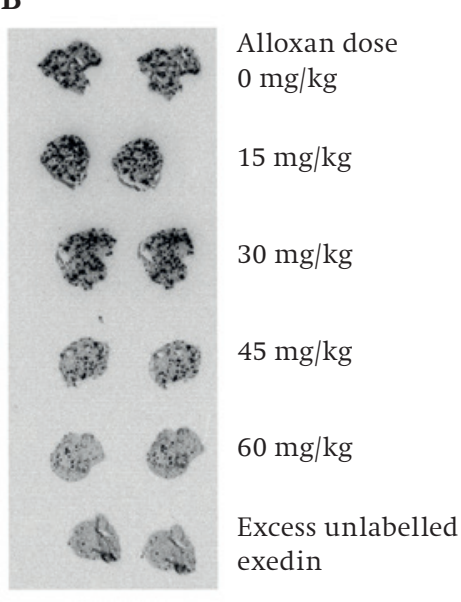

C
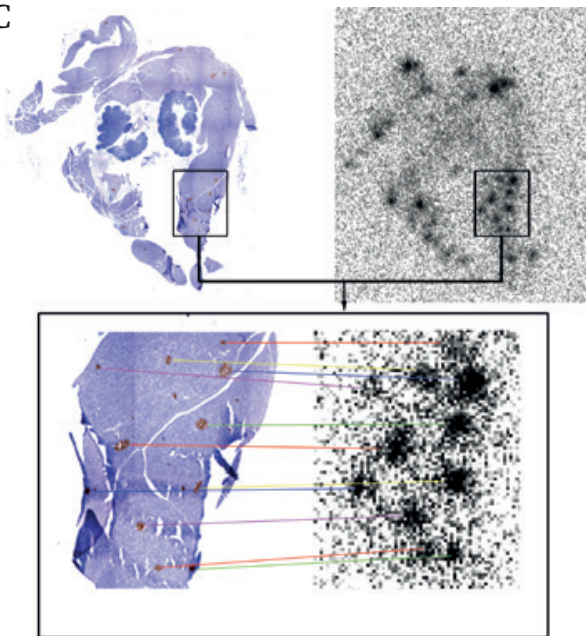

F
$\mathrm{D}$

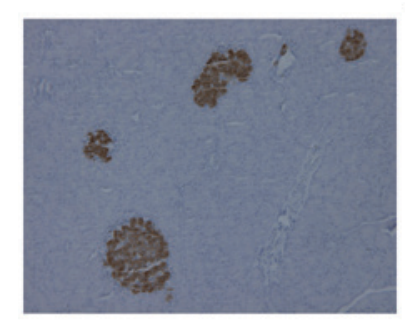

E

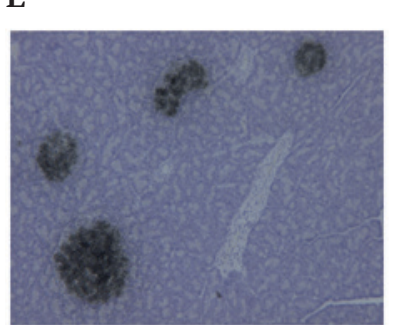

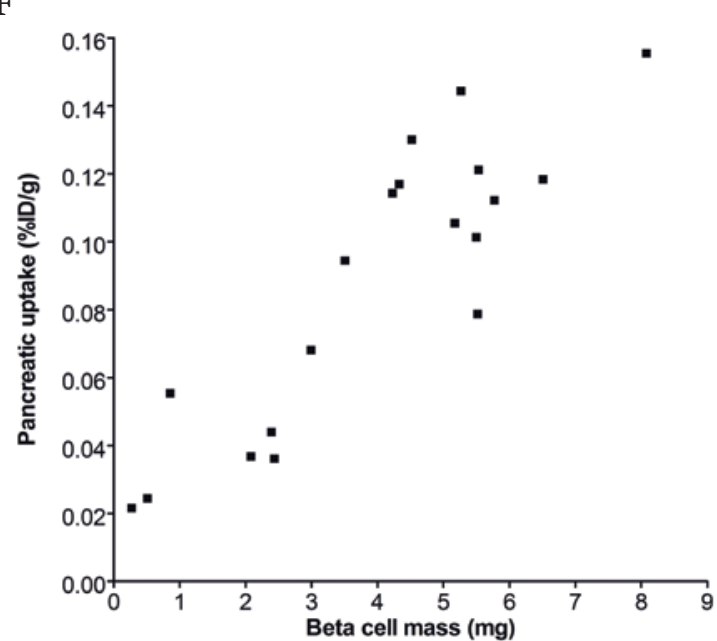

Figure 2 Continued.

Immunohistochemical staining of the pancreatic sections with an anti-insulin $\mathrm{mAb}$ showed colocalization of the stained islets and the hot-spots in autoradiography (Fig. 2C), demonstrating specific accumulation in the islets. Microautoradiography (Fig. 2D) also showed islet specific tracer uptake that colocalized with immunohistochemical staining of a consecutive section with an anti-insulin $\mathrm{mAb}$ (Fig. 2E) 


\section{SPECT of dissected tissues}

To visualize the uptake of ${ }^{11}$ In-exendin in the abdominal organs, SPECT scans of dissected organs in the peritoneal cavity of control and alloxan-treated Brown Norway rats were acquired (Fig. 3). The uptake of ${ }^{111}$ In-exendin in the pancreata of untreated Brown Norway rats were clearly visualized, whereas the pancreata of alloxan-treated rats as well as those from rats coinjected with an excess unlabelled exendin were barely visible by ex vivo SPECT (Fig. 3A-C). The resolution of the SPECT scanner is $600 \mu \mathrm{m}$, so individual islets of Langerhans could not be visualized (Fig. 3D). Blocking with an excess of unlabelled exendin resulted in a very low pancreatic uptake similar to the uptake in severely diabetic rats. Fig. 3E-F shows the ex vivo SPECT images of the dissected gastro-intestinal tract, spleen, liver and pancreas after injection of ${ }^{111}$ In-exendin. A strong signal was observed in the pancreas, whereas other abdominal organs showed only low uptake except for the proximal part of the duodenum and the distal part of the stomach that showed relatively high uptake.

\section{SPECT and quantitative analysis of SPECT images of diabetic rats}

The SPECT images of dissected tissues enabled accurate interpretation of the SPECT images of Brown Norway rats (Fig. 3G). In healthy Brown Norway rats, uptake in the pancreas was visible in SPECT scans after injection of ${ }^{111}$ In-exendin.

A
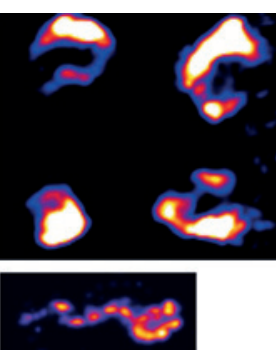
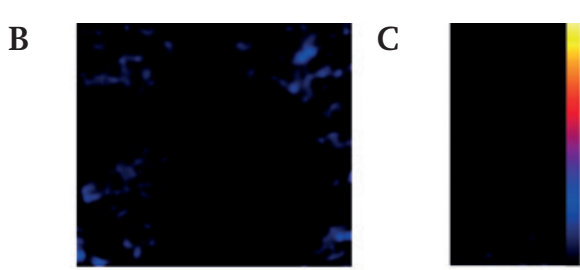

Figure 3 Ex vivo SPECT images of pancreata of control (A) and alloxan-treated Brown Norway rats (B). Blocking (C) was performed with an excess of unlabelled exendin $(25 \mu \mathrm{g})$ in 2 rats. Ex vivo SPECT images of the gastrointestinal tract of Brown Norway rats (E-F) and a high resolution SPECT scan of a pancreas (D). ${ }^{111}$ In-exendin-SPECT scans of Brown Norway rats with different amounts of BCM $(G)$ (acquired $1 \mathrm{~h}$ p.i.). Pancreata are indicated with red circles. H. Correlation between ${ }^{111}$ In-exendin uptake determined by quantitative analysis of SPECT scans and BCM (uptake on $\mathrm{y}$-axis in Becquerel, BCM in mg on $\mathrm{x}$-axis as determined by morphometric analysis after immunohistochemical staining with anti-insulin antibody). The correlation as determined by Pearson test is $r=0.83$.

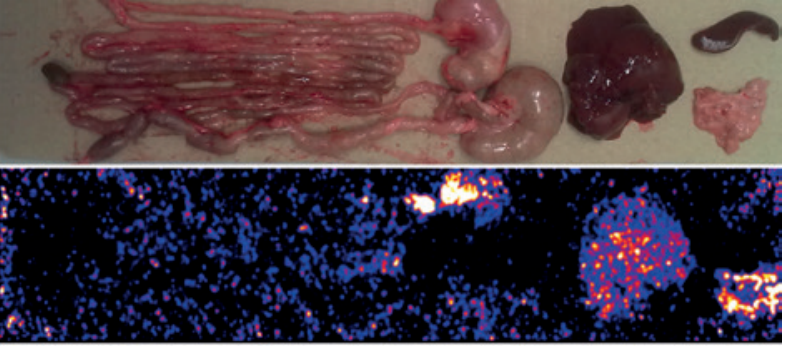

G

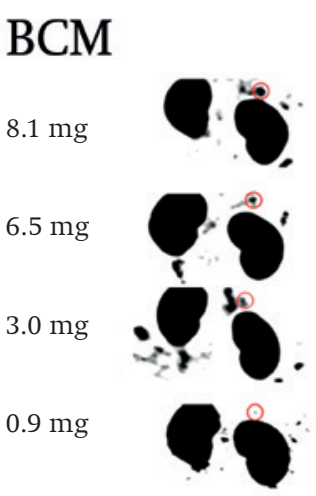

igure 3 Continued.

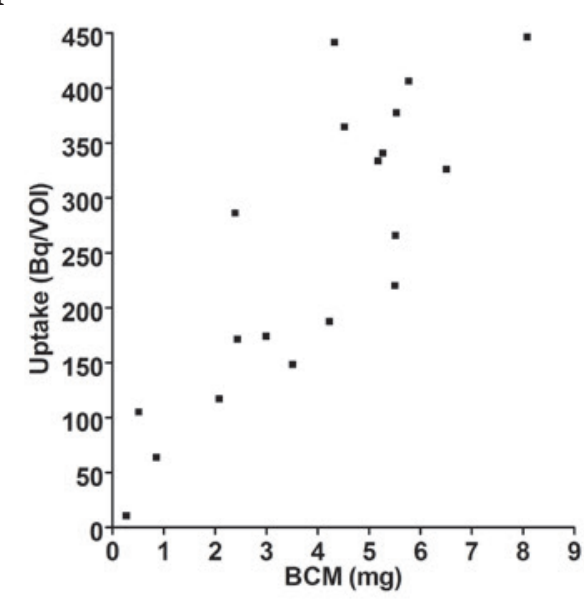

In diabetic rats there was negligible uptake in the pancreatic region and lowe uptake was observed in rats with reduced BCM. Besides high pancreatic uptake, uptake was also observed in the kidneys and lung. Moreover, SPECT images after injection of ${ }^{99 \mathrm{~m}} \mathrm{Tc} \mid$ Demobesin 3, specifically accumulating in the pancreas (20), correlated with the pancreatic uptake of ${ }^{11}$ In-exendin (Figure 1), confirming that the VOI used for quantification of BCM is indeed located in the pancreas. As previously described, the pancreas is the organ with the highest uptake of the bombesin analogue in the peritoneal cavity of rats (29), due efficient binding to the gastrin-releasing peptide receptor (GRP-R) that is abundantly expressed in the pancreas $(30,31)$. Removal of both kidneys enabled more accurate visualization of the pancreas, showing exact colocalization of [99m Tc]Demobesin and ${ }^{111} \mathrm{In}$-exendin as an additional proof that the VOI used for quantification contained the pancreas (Figure 1). The tracer uptake of ${ }^{111}$ In-exendin as determined by quantitative analysis of the SPECT images correlates linearly with the BCM determined by morphometric analysis (Fig. 3H, Pearson r=0.83) 


\section{Quantitative PCR}

In order to determine the GLP-1R mRNA expression in human and rat tissues, quantitative RT-PCR was performed. In rats, a 13-fold higher GLP-1R mRNA expression in the endocrine pancreas compared to the exocrine pancreas was found (Fig. 4A). The quantitative PCR on human tissues (Fig. 4B) showed high expression of the GLP-1R mRNA in the endocrine pancreas and very low expression in the exocrine pancreas with an endocrine-exocrine ratio of 47 . These results indicate that the GLP-1R expression profile is even more favourable in humans than in rats.
A
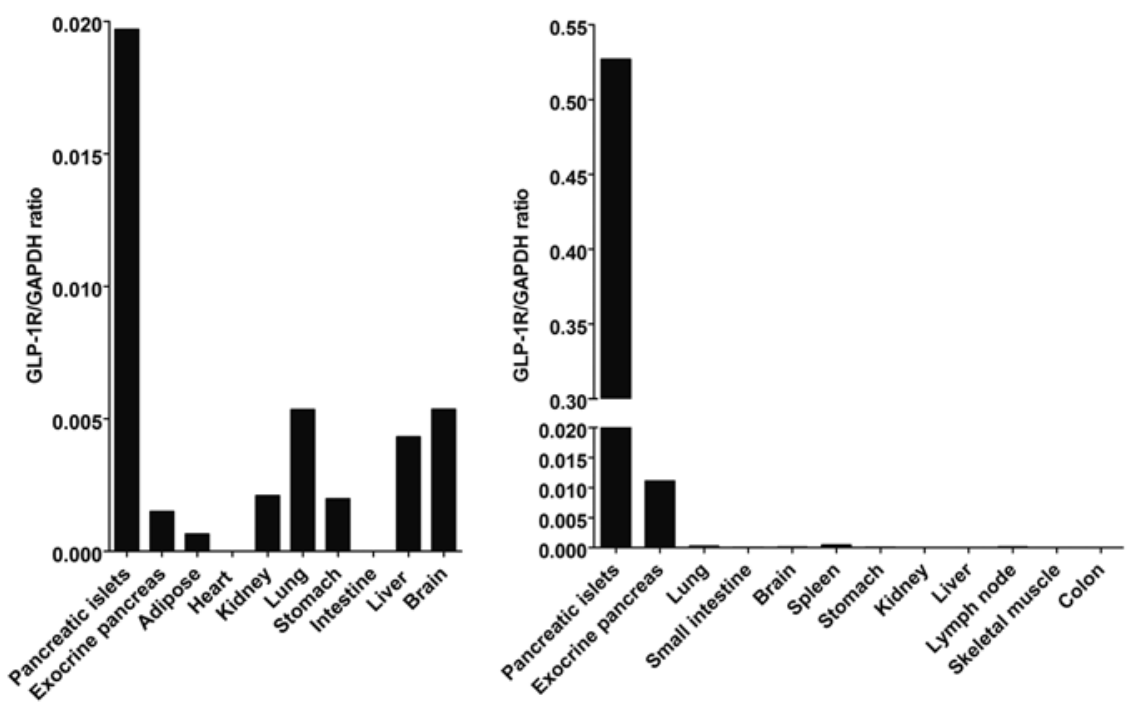
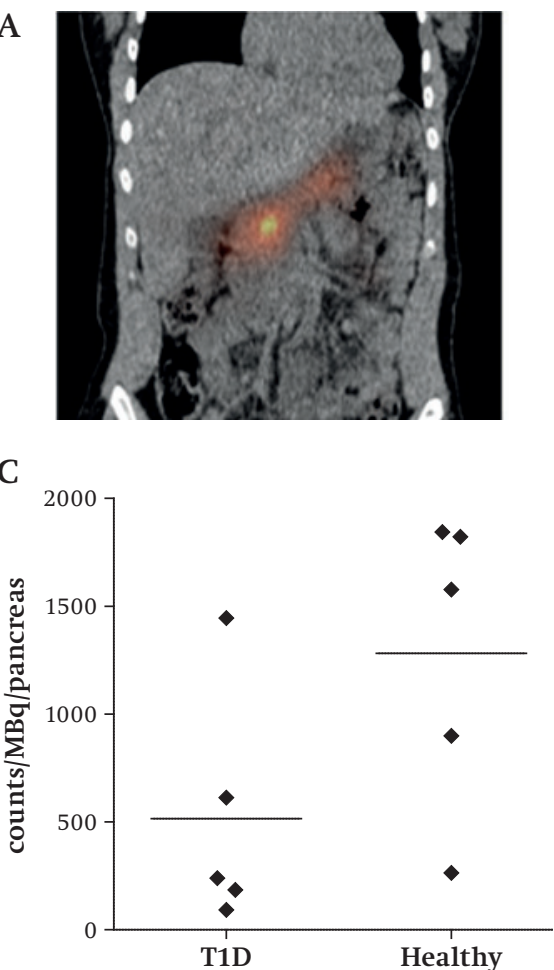
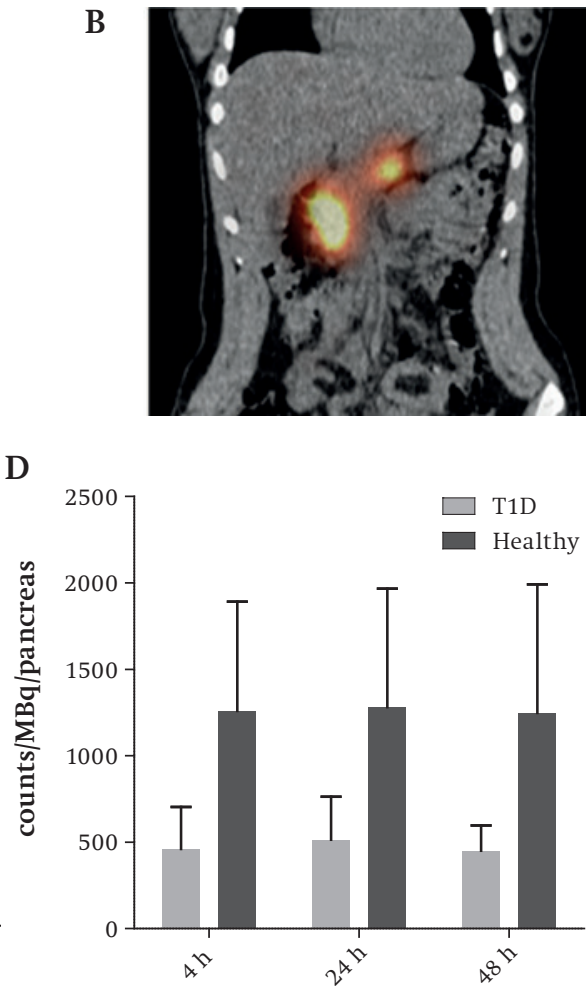

Figure 5 In vivo SPECT/CT images of the abdomen in humans, acquired $24 \mathrm{~h}$ after injection of ${ }^{11}$ In-exendin. Coronal cross-sections of a T1D patient (A) and a healthy volunteer (B), both show high uptake in the pancreas. Pancreatic uptake of ${ }^{111}$ In-Exendin quantified in SPECT images of 5 T1D patients and 5 healthy volunteers (C). Uptake was corrected for administered activity (142-153 MBq) and time after injection (23.2-24.7 h), resulting in pancreas uptake in total counts. The lines indicate the mean uptake per group. Mean and standard deviation of the pancreas activity per group, per scan moment (4 h, $24 \mathrm{~h}$ and $48 \mathrm{~h}$ post injection) (D).

\section{SPECT/CT in humans}

We explored the feasibility of the technology to determine the BCM in vivo in T1D patients $(\mathrm{n}=5)$ and matching healthy controls $(\mathrm{n}=5) .{ }^{111} \mathrm{In}$-exendin uptake is clearly visible in the human pancreas, as shown in Figure 5A and 5B. The pancreatic uptake of ${ }^{111}$ In-exendin was clearly decreased in T1D patients (Fig. 5C) with marked differences between T1D patients and healthy volunteers. Nevertheless, there was some overlap in pancreatic uptake of ${ }^{111}$ In-exendin between the groups. There was no significant difference in pancreatic uptake of ${ }^{111} \mathrm{In}$-exendin at 4,24 or $48 \mathrm{~h}$ after injection of ${ }^{111}$ In-exendin (Fig. 5D).

\section{Discussion}

In this study, we systematically characterized ${ }^{111}$ In-exendin in rats for the non-invasive determination of the pancreatic BCM by SPECT. The key findings of these studies are: (I) There is specific GLP-1R-mediated uptake of ${ }^{111}$ In-exendin in the pancreas; (II) ${ }^{111}$ In-exendin localizes specifically in the islets of Langerhans of rats; (III) the pancreatic uptake of ${ }^{111}$ In-exendin determined by ex vivo counting correlates 
linearly with BCM (Pearson $\mathrm{r}=0.89$ ); (IV) rats treated with high doses of alloxan, representing a controlled model of severe beta cell loss, showed an $80 \%$ reduction of tracer uptake in the pancreas indicating that even small differences in BCM can be detected; (V) the pancreatic tracer uptake decreased with BCM and only minima uptake was observed in rats treated with high doses of alloxan; (VI) most importantly, the pancreatic tracer uptake could be visualized by in vivo SPECT and quantitative analysis of the SPECT data correlated with the BCM in a linear manner (Pearson $\mathrm{r}=0.83$ ).

We have previously shown that the peptide dose is a critical factor that determines the uptake of ${ }^{111}$ In-exendin in GLP-1R positive tissues (32). It should be noted that the specific and high uptake of the tracer in the pancreas in this rat model is only observed at peptide doses not exceeding $0.1 \mu$ g per rat, as at higher peptide doses the GLP-1R in the pancreas are saturated as shown in the in vivo blocking experiments. Therefore, a tracer with a very high specific activity is required for SPECT (32). We have therefore improved the specific activity of ${ }^{111}$ In-exendin up to $700 \mathrm{MBq} / \mathrm{nmol}$, which allows administration of an activity dose sufficient for SPECT without exceeding the tracer dose of $0.1 \mu \mathrm{g} / \mathrm{rat}$

Previous preclinical studies with exendin labelled with ${ }^{18} \mathrm{~F}$ or ${ }^{64} \mathrm{Cu}$ have demonstrated their ability to visualise transplanted islets and subcutaneous tumours in rodents $(18,19)$. For the GLP-1R antagonist exendin-(9-39) labelled with ${ }^{18} \mathrm{~F}$, however, no correlation of uptake and pancreatic BCM could be demonstrated in a rat model (33). This is probably due to the fact that the antagonist exendin-(9-39) is not suitable for in vivo targeting of the GLP-1R (34), indicating that agonistic activity is required for successful visualisation of beta cells in vivo. Importantly, Reiner et al. have recently shown the in vivo targeting of beta cells with a fluorescent labelled exendin-4 analogue (35).

Previous studies suggested that GLP-1R expression is detected in the exocrine pancreas by in vitro autoradiography with ${ }^{125} \mathrm{I}-\mathrm{GLP}-1$ and immunohistochemistry on human pancreatic sections $(13,36)$. Our ex vivo autoradiographic analysis showed scattered focal hotspots throughout the pancreas which colocalized with the islets in immunohistochemical staining and only low background activity in the exocrine pancreas. This focal uptake decreased with alloxan-induced loss of beta cell mass and was almost completely absent in severely diabetic rats. The results of our ex vivo autoradiography are in line with the quantitative PCR analysis on rat tissues: high GLP-1R expression was observed in the endocrine pancreas, while low expression was apparent in the exocrine pancreas. The differences observed between our results and the previously published data $(13,36)$ may be explained by differences between binding characteristics of ${ }^{125}$ I-GLP-1 and GLP-1R antibodies in an in vitro assay as compared to the binding characteristics of ${ }^{111}$ In-exendin in vivo. Of note, in vivo ${ }^{111}$ In-exendin shows also internalization and metabolic trapping (37), which makes this technology highly sensitive and specific for the detection of GLP-1R positive tissues. In addition, artefacts potentially occurring in vitro leading to non-specific binding are avoided.

It was previously described that the GLP-1R expression is decreased in pancreatic islets in hyperglycaemic rats after near total pancreatectomy or constant glucose infusion (38), as determined by immunohistochemical staining and qPRC.

Our data show a strong correlation of tracer uptake and BCM under euglycemic as well as severe hyperglycaemic conditions. This observation argues against changes in GLP-1R expression that have significant influence of ${ }^{111}$ In-Exendin uptake. In addition, quantitative RT-PCR showed that the endocrine-exocrine GLP-1R expression ratio is even higher in humans than in rats (endocrine-exocrine ratio 47 in humans vs. 13 in rats). Of note, we cannot exclude some contamination of the exocrine pancreas with insulin producing cells, so that the actual endocrine exocrine ratio of GLP-1R mRNA expression may even be higher.

After these promising preclinical findings, we explored the feasibility of this technology to determine the BCM in vivo in T1D patients $(\mathrm{n}=5)$ and matching healthy controls $(\mathrm{n}=5)$. The pancreatic uptake of ${ }^{111}$ In-exendin showed considerable inter-individual variation, in line with variations in BCM as reported in the literature $(12,39)$. The pancreatic uptake of ${ }^{111}$ In-exendin was clearly decreased in T1D patients with marked differences between T1D patients and healthy volunteers. To date, no other radiotracer tested for BCM determination has shown such large maximum differences between healthy volunteers and T1D patients, nor has such a high inter-individual variability of the uptake been reported previously (40-47). A recent study in healthy volunteers and T1D patients with PET after injection of ${ }^{18} \mathrm{~F}$-Fluoropropyl-Dihydrotetrabenazine $\left({ }^{18} \mathrm{~F}-\mathrm{FP}-(+)-\mathrm{DTBZ}\right)$ showed a maximum difference of pancreatic uptake (represented in SUV) of $<40 \%$ (48) (Figure $4 \mathrm{~A}$ and $5 \mathrm{~A}$ of this reference). The difference in binding potential between T1D patients and healthy volunteers was not greater than 50\% (48). Moreover, recent publication showed that expression of VMAT2 in pancreatic polypeptide cells causes overestimation of the BCM in T1D with PET measurements (49). In thi study, the authors demonstrated that PP cells express VMAT2 and this contributes to the total pancreatic uptake of ${ }^{18} \mathrm{~F}-\mathrm{FP}-(+)-\mathrm{DTBZ}$. These data are in line with the reduction of pancreatic uptake of ${ }^{18} \mathrm{~F}-\mathrm{FP}-(+)-\mathrm{DTBZ}$ by less than $40 \%$ in T1D patients. The considerably higher differences in pancreatic uptake between healthy subjects and T1D patients point towards a considerably higher specificity of ${ }^{111}$ In-exendin for beta cells.

Of note, the dynamic scanning protocol described including arterial blood sampling makes this a laborious procedure. Our SPECT protocol with ${ }^{111}$ In-exendin is a much simpler method that requires a single injection of the radiotrace followed by single time point static SPECT acquisition and subsequent quantitative 
analysis of the images. In addition, the method can be used in every hospita equipped with a gamma camera and does not require a cyclotron. Also, the labeled compound can easily be distributed ready to use for clinical studies.

In summary, we here demonstrate in a preclinical imaging model of beta cell loss that the uptake of ${ }^{111}$ In-exendin, as determined by in vivo imaging, is specific fo the beta cells and shows a direct correlation with BCM. In humans, we observed considerable inter-individual differences in pancreatic tracer uptake (in line with available autopsy data) and a marked reduction of pancreatic uptake in T1D patients. To the best of our knowledge, no comparable preclinical evidence has been created for any other radiotracer tested for in vivo imaging of BCM with respect to beta cell-specificity and correlation of uptake to actual BCM so far. Of note, clinical imaging does not require complicated dynamic scanning techniques or calculation of binding capacity, making it feasible for efficient use in large clinical trials (19). Therefore, our data indicate that this technology could indeed be the first to enable non-invasive determination of BCM in humans, offering a great potential to better elucidate the pathophysiology of the different types and stages of diabetes.

\section{Acknowledgements}

We thank Dr. Theodosia Maina and Dr. Berthold Nock at the National Centre for Scientific Research “Demokritos”, Athens, Greece for kindly providing Demobesin-3 and Marleen Melis, Jan de Swart and Erik de Blois at the Erasmus Medical Centre, Rotterdam, The Netherlands for technical support of the SPECT experiments. Heleen de Wit (Department of Internal Medicine, Radboud university medica center, Nijmegen, The Netherlands) assisted in the recruitment of patients. We acknowledge Willem Grootjans (Department of Nuclear Medicine, Radboud university medical center, Nijmegen, The Netherlands) for technical assistance in image analysis of the patient data.

\section{Funding}

Our work was supported by NIH grant 1R01 AG 030328-01 and the European

Community's Seventh Framework Programme (FP7/2007-2013) project BetaImage, under grant agreement nº 222980

\section{References}

Diagnosis and classification of diabetes mellitus. Diabetes care. 2006;29 Suppl 1:S43-8.

2. Lohr M, Kloppel G. Residual insulin positivity and pancreatic atrophy in relation to duration of chronic type 1 (insulin-dependent) diabetes mellitus and microangiopathy. Diabetologia. 1987;30(10):757-62

3. Steele C, Hagopian WA, Gitelman S, Masharani U, Cavaghan M, Rother KI, et al. Insulin secretion in type 1 diabetes. Diabetes. 2004;53(2):426-33.

4. Coppieters KT, Dotta F, Amirian N, Campbell PD, Kay TW, Atkinson MA, et al. Demonstration of is let-autoreactive CD8 T cells in insulitic lesions from recent onset and long-term type 1 diabetes patients. The Journal of experimental medicine. 2012;209(1):51-60. Epub 2012/01/04.

5. Klinke DJ, 2nd. Extent of beta cell destruction is important but insufficient to predict the onset of type 1 diabetes mellitus. PloS one. 2008;3(1):e1374. Epub 2008/01/03.

6. Couri CE, Oliveira MC, Stracieri AB, Moraes DA, Pieroni F, Barros GM, et al. C-peptide levels and insulin independence following autologous nonmyeloablative hematopoietic stem cell transplantation in newly diagnosed type 1 diabetes mellitus. JAMA : the journal of the American Medical Association. 2009;301(15):1573-9. Epub 2009/04/16.

7. DeFronzo RA. Pathogenesis of type 2 diabetes: metabolic and molecular implications for identifying diabetes genes. Diabetes Reviews. 1997;5(3):177-269.

8. Taylor SI. Deconstructing type 2 diabetes. Cell. 1999;97(1):9-12

Weir GC, Bonner-Weir S. Five stages of evolving beta-cell dysfunction during progression to diabetes Diabetes. 2004;53 Suppl 3:S16-21. Epub 2004/11/25

10. Cnop M, Welsh N, Jonas JC, Jorns A, Lenzen S, Eizirik DL. Mechanisms of pancreatic beta-cell death in type 1 and type 2 diabetes: many differences, few similarities. Diabetes. 2005;54 Suppl 2:S97-107.

11. Butler AE, Janson J, Bonner-Weir S, Ritzel R, Rizza RA, Butler PC. Beta-cell deficit and increase beta-cell apoptosis in humans with type 2 diabetes. Diabetes. 2003,52(1).102-10.

2. Ritzel RA, Butler AE, Rizza RA, Veldhuis JD, Butler PC. Relationship between beta-cell mass an fasting blood glucose concentration in humans. Diabetes care. 2006;29(3):717-8. Epub 2006/03/01,

3. Korner M, Stockli M, Waser B, Reubi JC. GLP-1 receptor expression in human tumors and human normal tissues: potential for in vivo targeting. J Nucl Med. 2007;48(5):736-43.

4. Tornehave D, Kristensen P, Romer J, Knudsen LB, Heller RS. Expression of the GLP-1 receptor in mouse, rat, and human pancreas. J Histochem Cytochem. 2008;56(9):841-51

15. Brom M, Oyen WJ, Joosten L, Gotthardt M, Boerman OC. 68Ga-labelled exendin-3, a new agent for the detection of insulinomas with PET. European journal of nuclear medicine and molecular imaging. 2010;37(7):1345-55. Epub 2010/01/30

16. Wang Y, Lim K, Normandin M, Zhao XJ, Cline GW, Ding YS. Synthesis and evaluation of [F-18]exendin (9-39) as a potential biomarker to measure pancreatic beta-cell mass. Nuclear medicine and biology. 2012;39(2):167-76.

17. Wild D, Wicki A, Mansi R, Behe M, Keil B, Bernhardt P, et al. Exendin-4-based radiopharmaceuticals for glucagonlike peptide-1 receptor PET/CT and SPECT/CT. J Nucl Med. 2010;51(7):1059-67. Epub 2010/07/03.

18. Wu Z, Liu S, Hassink M, Nair I, Park R, Li L, et al. Development and evaluation of 18F-TTCO-Cys40-Exendin-4. a PET probe for imaging transplanted islets.J Nucl Med. 2013;54(2).244-51. Epub 2013/01/09.

19. Wu Z, Todorov I, Li L, Bading JR, Li Z, Nair I, et al. In vivo imaging of transplanted islets with 64CuDO3A-VS-Cys40-Exendin-4 by targeting GLP-1 receptor. Bioconjugate chemistry. 2011;22(8):1587-94. Epub 2011/06/23.

20. Nock BA, Nikolopoulou A, Galanis A, Cordopatis P, Waser B, Reubi JC, et al. Potent bombesin-like peptides for GRP-receptor targeting of tumors with $99 \mathrm{mTC}$ a preclinical study. Journal of medicina chemistry. 2005;48(1):100-10.

21. Heskamp S, van Laarhoven HW, Molkenboer-Kuenen JD, Franssen GM, Versleijen-Jonkers YM, Oyen WJ, et al. ImmunoSPECT and immunoPET of IGF-1R expression with the radiolabeled antibody R1507 in a triple-negative breast cancer model. J Nucl Med.51(10):1565-72. 
22. Rademakers SE, Rijken PF, Peeters WJ, Nijkamp MM, Barber PR, Laak Jvd, et al. Parametric mapping of mmunohistochemically stained tissue sections; a method to quantify the colocalization of tumor markers. submitted.

23. Rijken PF, Bernsen HJ, van der Kogel AJ. Application of an image analysis system to the quantitation of tumor perfusion and vascularity in human glioma xenografts. Microvascular research. 1995:50(2): $141-53$.

24. Garofano A, Czernichow P, Breant B. Beta-cell mass and proliferation following late fetal and early postnatal malnutrition in the rat. Diabetologia. 1998;41(9):1114-20.

25. Chen CM, Behringer RR. Cloning, structure, and expression of the mouse Ovca1 gene. Biochemical and biophysical research communications. 2001:286(5):1019-26.

26. Cunha DA, Hekerman P, Ladriere L, Bazarra-Castro A, Ortis F, Wakeham MC, et al. Initiation and execution oflinotoxic ER stress in pancreatic beta-cells. Journal of cell science 2008:121(Pt14) $2308-18$.

7. Overbergh L Valckx D, Waer M, Mathieu C. Ountification of murine cytokine mRNAs using real

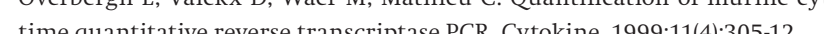

28. Report of the Expert Committee on the Diagnosis and Classification of Diabetes Mellitus. Diabetes care. 1997;20(7):1183-97. Epub 1997/07/01.

29. Maina T, Nock BA, Zhang H, Nikolopoulou A, Waser B, Reubi JC, et al. Species differences of bombesin analog interactions with GRP-R define the choice of animal models in the development of GRP-R-targeting drugs. J Nucl Med. 2005;46(5):823-30.

30. Jensen RT, Moody T, Pert C, Rivier JE, Gardner JD. Interaction of bombesin and litorin with specific membrane receptors on pancreatic acinar cells. Proceedings of the National Academy of Sciences of the United States of America. 1978;75(12):6139-43

31. Moran TH, Moody TW, Hostetler AM, Robinson PH, Goldrich M, McHugh PR. Distribution of bombesin binding sites in the rat gastrointestinal tract. Peptides. 1988;9(3):643-9.

32. Brom M, Oyen WJ, Joosten L, Gotthardt M, Boerman OC. 68Ga-labelled exendin-3, a new agent for the detection of insulinomas with PET. European journal of nuclear medicine and molecular imaging $37(7): 1345-55$

33. Wang Y, Lim K, Normandin M, Zhao X, Cline GW, Ding YS. Synthesis and evaluation of [18F]exendin (9-39) as a potential biomarker to measure pancreatic beta-cell mass. Nuclear medicine and biology. 2012;39(2):167-76. Epub 2011/10/29.

34. Brom M, Joosten L, Oyen WJ, Gotthardt M, Boerman OC. Radiolabelled GLP-1 analogues for in vivo targeting of insulinomas. Contrast media \& molecular imaging. 2012;7(2):160-6. Epub 2012/03/22.

35. Reiner T, Thurber G, Gaglia J, Vinegoni C, Liew CW, Upadhyay R, et al. Accurate measurement of pancreatic islet \{beta\}-cell mass using a second-generation fluorescent exendin-4 analog. Proceeding of the National Academy of Sciences of the United States of America.108(31):12815-20.

36. Xu G, Kaneto H, Lopez-Avalos MD, Weir GC, Bonner-Weir S. GLP-1/exendin-4 facilitates beta-cell neogenesis in rat and human pancreatic ducts. Diabetes research and clinical practice. 2006;73(1):107-10. 37. Brom M, Andralojc K, Oyen WJ, Boerman OC, Gotthardt M. Development of radiotracers for the determination of the beta-cell mass in vivo. Current pharmaceutical design. 2010;16(14):1561-7. Epub $2010 / 02 / 12$.

38. Xu G, Kaneto H, Laybutt DR, Duvivier-Kali VF, Trivedi N, Suzuma K, et al. Downregulation of GLP-1 and GIP receptor expression by hyperglycemia - Possible contribution to impaired incretin effects in diabetes. Diabetes. 2007;56(6):1551-8.

39. Rahier J, Guiot Y, Goebbels RM, Sempoux C, Henquin JC. Pancreatic beta-cell mass in European subjects with type 2 diabetes. Diabetes, obesity \& metabolism. 2008;10 Suppl 4:32-42. Epub 2008/10/18.

4. Moore A, Bonner-Weir S, Weissleder R. Noninvasive in vivo measurement of beta-cell mass in mouse model of diabetes. Diabetes. 2001;50(10):2231-6. Epub 2001/09/28.

41. Freeby M, Goland R, Ichise M, Maffei A, Leibel R, Harris P. VMAT2 quantitation by PET as a biomarker for beta-cell mass in health and disease. Diabetes, obesity \& metabolism. 2008;10 Suppl 4:98-108. Epub $2008 / 10 / 18$
42. Goland R, Freeby M, Parsey R, Saisho Y, Kumar D, Simpson N, et al. 11C-dihydrotetrabenazine PET of the pancreas in subjects with long-standing type 1 diabetes and in healthy controls. J Nucl Med. 2009;50(3):382-9.

43. de Lonlay P, Simon-Carre A, Ribeiro MJ, Boddaert N, Giurgea I, Laborde K, et al. Congenital hyperinsulinism: pancreatic [18F]fluoro-L-dihydroxyphenylalanine (DOPA) positron emission tomography and immunohistochemistry study of DOPA decarboxylase and insulin secretion. The Journal of clinical endocrinology and metabolism. 2006;91(3):933-40.

44. Hardy OT, Hernandez-Pampaloni M, Saffer JR, Suchi M, Ruchelli E, Zhuang H, et al. Diagnosis an localization of focal congenital hyperinsulinism by 18F-fluorodopa PET scan. The Journal of pediatrics. 2007;150(2):140-5.

45. Otonkoski T, Nanto-Salonen K, Seppanen M, Veijola R, Huopio H, Hussain K, et al. Noninvasive diagnosi of focal hyperinsulinism of infancy with [18F]-DOPA positron emission tomography. Diabetes. 2006 55(1):13-8.

46. Rubi B, Ljubicic S, Pournourmohammadi S, Carobbio S, Armanet M, Bartley C, et al. Dopamine D2-like receptors are expressed in pancreatic beta cells and mediate inhibition of insulin secretion. The Journal of biological chemistry. 2005;280(44):36824-32.

47. Sweet IR, Cook DL, Lernmark A, Greenbaum CJ, Wallen AR, Marcum ES, et al. Systematic screening of potential beta-cell imaging agents. Biochemical and biophysical research communications. 2004; 314(4):976-83.

48. Normandin MD, Petersen KF, Ding YS, Lin SF, Naik S, Fowles K, et al. In Vivo Imaging of Endogenous Pancreatic beta-Cell Mass in Healthy and Type 1 Diabetic Subjects Using F-18-Fluoropropyl-Dihydrotetrabenazine and PET. Journal of Nuclear Medicine. 2012:53(6):908-16.

49. Freeby M, Ichise M, Harris PE. Vesicular monoamine transporter, type 2 (VMAT2) expression as it compares to insulin and pancreatic polypeptide in the head, body and tail of the human pancreas. Islets. 2012:4(6):393-7. Epub 2012/12/12. 


\section{5}

Quantified pancreatic uptake of ${ }^{111}$ In-exendin in patients with type 1 diabetes and healthy controls

Wietske Woliner - van der Weg, Maarten Brom, Martin Béhe, Cees J. Tack, Otto C. Boerman, Marcel J.R. Janssen, Martin Gotthardt 


\section{Abstract}

Determination of changes in pancreatic beta cell mass (BCM) during development and treatment of diabetes, and studying the relation between BCM and beta cell function, require a non-invasive test for quantification of the pancreatic BCM. Beta cells express the glucagon-like peptide-1 (GLP-1) receptor, which is specifically targeted by exendin. Therefore pancreatic uptake of ${ }^{111}$ In-exendin, determined by quantitative single photon emission computed tomography (SPECT), could be used as an imaging biomarker for the BCM. As a first step towards clinical use of ${ }^{111}$ In-exendin for non-invasive BCM quantification, we acquired and analyzed ${ }^{111}$ In-exendin-SPECT images in 10 type 1 diabetes (T1D) patients and 10 matched healthy controls.

Pancreatic uptake of ${ }^{111}$ In-exendin was on average three times lower in T1D patients than in the healthy controls (631 vs 1813 counts per MBq (c/MBq)). Visual and quantitative analysis demonstrated a wide interindividual variation of pancreatic uptake within both groups (T1D: range 107-1532 c/MBq, healthy volunteers: 442-2298 c/MBq). The amount of ${ }^{111}$ In in the pancreas (corrected for decay) remained stable for 7 days. Uptake decreased with the duration of T1D.

These results suggest that quantification of ${ }^{111}$ In-exendin uptake is a method for in vivo $\mathrm{BCM}$ quantification. The method for $\mathrm{BCM}$ quantification demonstrated here is simple to use and can be applied in virtually any nuclear medicine department, using the ready to use kit formulation applied here. Therefore, this imaging method could be highly useful to further elucidate the complex pathophysiology of diabetes.

ClinicalTrials.gov Identifier: NCT01825148

\section{Introduction}

Beta cell dysfunction and death are key events in the pathogenesis of T1D and T2D but to date only incomplete information is available on the natural history of los of beta cell mass (BCM) as compared to beta cell function. This is due to the fact that characterization of beta cells is limited to pancreatic specimens that can only be obtained at autopsy (pancreatic biopsy in vivo is associated with an inacceptable high rate of complications including death) (1). As a consequence, the curren treatment of diabetes is empirical, aiming to correct hyperglycaemia representing a symptom being the consequence of the underlying pathophysiological process. Therefore, most current treatments may not prevent progressive beta cell loss or dysfunction as they are following symptoms arising from the progress of the disease. Individual response to treatment of diabetes is difficult to predict because of the heterogeneous nature of the pathophysiology of the disease (2-4). These fact indicate that clinical imaging of beta cells is a prerequisite for understanding the natural history of beta cell dysfunction and loss in diabetes $(5,6)$. Understanding this key issue will allow developing innovative personalized precision medicine strategies for optimized diabetes prevention and treatment and will provide the required tools to evaluate efficiency of these novel therapies (7).

To date, several radiotracer molecules have been clinically evaluated for in vivo imaging of beta cells. Radiolabeled dihydrotetrabenazine (DTBZ) has been demonstrated to show lower uptake (as well as binding capacity) in pancreata of patients with T1D. However, the true role of this tracer in imaging in diabetes remains to be elucidated as it is not specific for beta cells (8). Despite the lack of specificity for beta cells, DTBZ could still play a role in patient stratification in diabetes. Recently, ${ }^{11} \mathrm{C}$-HTP (hydroxyl tryptophan) has been established as an imaging biomarker for the complete endocrine pancreas and lower uptake has been demonstrated in patients with T1D. As compared with DTBZ, the differences in pancreatic uptake between T1D patients and healthy volunteers were more pronounced with ${ }^{11} \mathrm{C}$-HTP $(9)$.

We have recently demonstrated that in vivo imaging of the GLP-1 receptor may reflect BCM. In a rodent model of beta cell loss, the uptake of radiolabeled exendin, specifically binding to the GLP-1 receptor (GLP-1R), directly correlated with BCM. In addition, the feasibility of in vivo imaging in humans has been demonstrated (10) Also, we have demonstrated that the uptake of radiolabeled exendin in the pancreas is not affected by the presence of alpha cells, which had been a concern regarding the specificity of the tracer for beta cells (11). Based on these observations and the fact that we demonstrated low uptake of ${ }^{111}$ In-Exendin in the exocrine pancreas as compared to the endocrine pancreas (10), there is considerable data for suggesting that radiolabeled exendin is indeed a specific imaging biomarker for BCM. 
Here, we report the results of the first clinical study in which pancreatic uptake of radiolabeled exendin has been evaluated in patients with long-standing T1D and compared to age-, BMI- and gender-matched healthy volunteers. We demonstrate large differences in pancreatic uptake between the two groups as well as a reduction of the uptake with duration of disease.

\section{Research design and methods}

\section{Patient inclusion}

We included 10 patients with T1D diagnosed at least 5 years before inclusion and 10 healthy volunteers having $\mathrm{HbA}_{1 \mathrm{C}}<7 \%(<53 \mathrm{mmol} / \mathrm{mol})$ and normal glucose tolerance. For creating two comparable groups, healthy controls were matched to T1D patients for BMI (maximum difference $2 \mathrm{~kg} / \mathrm{m}^{2}$ ), age (maximum difference 10 years) and gender.

Inclusion criteria were BMI between 18.5 and $27 \mathrm{~kg} / \mathrm{m}^{2}$, age 21-60 y, normal kidney function (MDRD $>40 \mathrm{~mL} / \mathrm{min}$ ) and no previous treatment with exenatide or dipeptidyl-peptidase IV inhibitors.

Glucose tolerance in healthy volunteers was assessed by an oral glucose tolerance test after fasting for at least 10 hours. Within 5 minutes, subjects had to drink $250 \mathrm{ml}$ of water, containing 75 gram of anhydrous glucose. For inclusion, the blood glucose 2 hours after glucose intake had to be below $7.8 \mathrm{mmol} / \mathrm{L}(12)$.

As a measure of beta cell function in T1D patients, non-stimulated C-peptide was measured after fasting for at least 4 hours. Thereafter, stimulated C-peptide was measured 6 minutes after stimulation with $1 \mathrm{mg}$ glucagon. The measured C-peptide values were not used to exclude participants.

The study was approved by the regional ethics review board (CMO region Arnhem-Nijmegen). All subjects had to sign written informed consent in accordance with provisions of the Declaration of Helsinki prior to inclusion.

\section{Preparation and administration of ${ }^{111} \ln$-exendin}

The ${ }^{111}$ In-exendin for this clinical study was prepared and labeled as described previously(10). Each patient received $150 \mathrm{MBq}{ }^{111}$ In-exendin-4 dissolved in $5 \mathrm{~m}$. $0.9 \% \mathrm{NaCl}$, corresponding to a peptide dose of $1 \mu \mathrm{g}$ exendin. ${ }^{111} \mathrm{In}$-exendin was administered as a slow bolus over 5 minutes after 4 hours of fasting (in order to reduce the potential influence of increased blood flow to the islets on radiotrace uptake). Glucose level and blood pressure were monitored for 4 hours after injection. Subjects were allowed to eat 2 hours after injection or earlier, in case of low blood glucose levels.

\section{Imaging}

In all subjects SPECT (single photon emission computed tomography) of the region of the pancreas and whole body planar images were acquired $4 \mathrm{~h}, 24 \mathrm{~h}$ and $48 \mathrm{~h}$ after administration of the radiotracer. For dosimetry purposes, 5 T1D patients and 4 matched healthy volunteers had additional planar imaging direct after administration, and SPECT and planar imaging at $96 \mathrm{~h}$ and $128 \mathrm{~h}$ after administration. Twenty-four hours after administration, a low-dose CT scan was acquired simultaneously with the SPECT scan for attenuation and scatter correction, and as anatomical reference. SPECT/CT acquisition and reconstruction was performed as previously described (10).

\section{Quantification of SPECT images}

Images were quantified with the previously described method (11). Briefly, the uptake of the tracer in the pancreas was calculated based on the counts within two spherical volumes of interest placed in the head and corpus region (on SPECT) multiplied by the volume of the pancreas (as measured on CT). Counts were corrected for administered activity and time after injection, leading to the pancreatic uptake in counts per MBq administered activity (c/MBq).

\section{Statistical analysis}

The two tailed Mann Whitney test was performed to compare the results of the T1D patients and the healthy volunteers and to compare the results at differen imaging time points. The Pearson's correlation coefficient was calculated for the time since diagnosis versus the pancreatic uptake, and age versus uptake in the T1D patients. For all tests $\mathrm{P}<0.05$ was considered significant. For the tests we used GraphPad Prism version 5.00 (GraphPad Software, San Diego California USA).

\section{Results}

\section{Patients}

Patient characteristics are provided in Table 1. The groups were well matched fo age, gender and BMI. In both the groups 4 men and 6 women were included.

CT based pancreas volumes (see table 1 ) were significantly smaller in the T1D patients $\left(\mathrm{P}=0.0005\right.$, range T1D $20.7-82.4 \mathrm{~cm}^{3}$, healthy volunteers $\left.65.5-136.3 \mathrm{~cm}^{3}\right)$. No severe side effects were reported, only a few T1D patients showed a fall in blood glucose, attributed to the ongoing action of long-acting insulin. Two patients had measurable, but still very low ( $\leq 0.06 \mathrm{nmol} / \mathrm{L})$ stimulated C-peptide levels (see table 1 ). 


\begin{tabular}{|c|c|c|c|c|c|c|c|c|c|}
\hline Subject-code $^{1}$ & Protocol $^{2}$ & Sex & Age (y) & Weight (kg) & BMI $\left(\mathrm{kg} / \mathrm{m}^{2}\right)$ & $\begin{array}{l}\text { Duration } \\
\text { T1D (y) }\end{array}$ & $\begin{array}{l}\text { Stimulated } \\
\text { C-peptide (nmol/L) }\end{array}$ & $\begin{array}{l}\text { CT based pancreas } \\
\text { volume }\left(\mathrm{cm}^{3}\right)\end{array}$ & $\begin{array}{l}\text { Uptake in the whole pancreas } 24 \mathrm{~h} \text { post injection } \\
\text { (counts/ MBq) }\end{array}$ \\
\hline D01 & Short & M & 50 & 78.0 & 26.1 & 30 & $<0.03$ & 60.9 & 388 \\
\hline D02 & Long & $\mathrm{F}$ & 25 & 53.2 & 21.3 & 13 & $<0.03$ & 65.7 & 1532 \\
\hline D03 & Long & M & 47 & 76.6 & 23.6 & 27 & $<0.03$ & 47.7 & 186 \\
\hline D04 & Long & $\mathrm{F}$ & 54 & 58.1 & 21.9 & 33 & $<0.03$ & 20.7 & 183 \\
\hline D05 & Short & F & 41 & 68.5 & 25.2 & 27 & $<0.03$ & 72.1 & 1079 \\
\hline D06 & Short & F & 53 & 56.2 & 22.0 & 41 & $<0.03$ & 25.4 & 119 \\
\hline D07 & Long & M & 21 & 89.3 & 26.1 & 7.0 & 0.04 & 82.4 & 763 \\
\hline D08 & Short & $\mathrm{F}$ & 51 & 67.1 & 23.8 & 15 & 0.06 & 60.0 & 1255 \\
\hline D09 & Long & $\mathrm{F}$ & 21 & 64.6 & 23.2 & 8.5 & $<0.03$ & 51.8 & 695 \\
\hline D10 & Short & M & 29 & 90.7 & 24.6 & 21 & $<0.03$ & 45.5 & 107 \\
\hline T1D: Average ( \pm STD) & & & $39.2( \pm 13.7)$ & $70.2( \pm 13.2)$ & $23.8( \pm 1.7)$ & $22.3( \pm 11.2)$ & & $53.2( \pm 19.4)$ & $630.7( \pm 518.3)$ \\
\hline H01 & Short & M & 54 & 82.3 & 24.3 & & & 88.8 & 508 \\
\hline H02 & Long & $\mathrm{F}$ & 24 & 55.3 & 19.8 & & & 73.1 & 2535 \\
\hline H03 & Long & M & 40 & 80.1 & 23.9 & & & 108.1 & 2156 \\
\hline H04 & Long & $\mathrm{F}$ & 54 & 58.9 & 22.4 & & & 73.8 & 1316 \\
\hline H05 & Short & F & 43 & 63.2 & 24.1 & & & 87.5 & 1729 \\
\hline H06 & Short & $\mathrm{F}$ & 50 & 72.3 & 23.1 & & & 94.4 & 2506 \\
\hline H07 & Long & M & 26 & 83.0 & 25.6 & & & 136.3 & 2110 \\
\hline H08 & Short & F & 49 & 71.9 & 24.9 & & & 86.2 & 2269 \\
\hline H09 & Long $^{3}$ & $\mathrm{~F}$ & 22 & 61.5 & 22.1 & & & 65.5 & 954 \\
\hline H10 & Short & M & 24 & 81.0 & 23.7 & & & 72.0 & 2046 \\
\hline Healthy: Average ( $\pm S T D)$ & & & $38.6( \pm 13.3)$ & $71.0( \pm 10.5)$ & $23.4( \pm 1.6)$ & & & $88.6( \pm 21.0)$ & $1812.9( \pm 680.2)$ \\
\hline
\end{tabular}

\section{Pancreatic uptake}

As shown in figure 1, in most subjects uptake in the pancreas can clearly be visualized on coronal cross-sections of the SPECT images. Visual analysis of the images demonstrated a wide variation in the uptake within both groups, which is confirmed by the quantification of the total pancreatic uptake.

The T1D patients had significantly fewer counts in the pancreas than the healthy volunteers $(\mathrm{p}=0.0011)$, with an average uptake of 631 counts/ MBq for the T1D patients (range 107-1532 counts/MBq) and of 1813 counts/MBq for the healthy volunteers (range 442-2298 counts/MBq) at $24 \mathrm{~h}$ post injection (Fig 1).

Also the average number of counts per voxel (activity concentration) in the volumes of interest in the pancreas, representing the concentration of ${ }^{111}$ In-exendin in the pancreas, showed a large variation within both groups. Also for this 'concentration', the difference between the groups is significant $(\mathrm{P}=0.0147)$. 


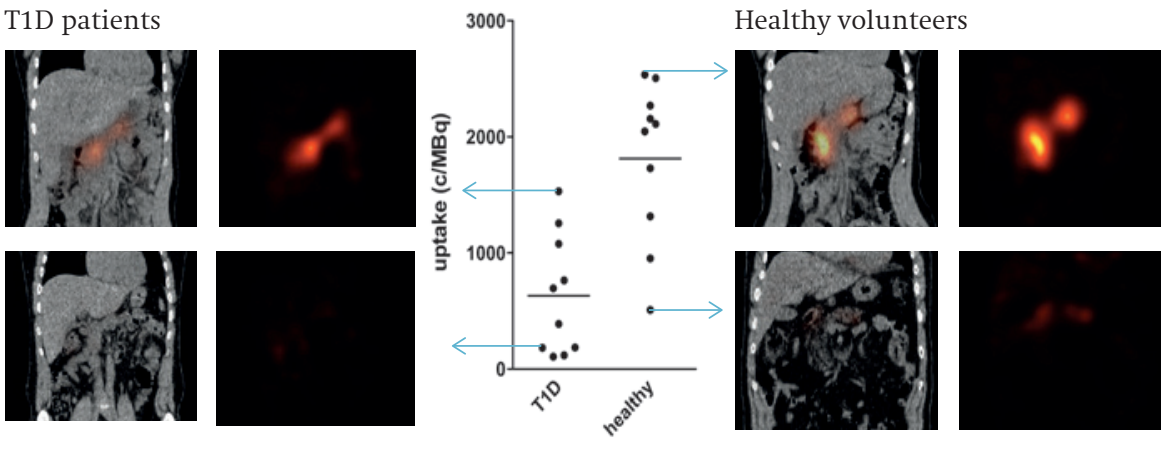

Figure 1 The graph shows the uptake of ${ }^{111}$ In-exendin (counts per MBq) in the whole pancreata of the individual subjects $24 \mathrm{~h}$ post injection. On the left side coronal cross sections of the SPECT-CT and SPECT of the T1D patients with the highest and lowest uptake. On the right side the images of the healthy volunteers with the highest and lowest uptake. The scaling of the SPECT images is identical in all images.

Comparing the activity concentration in the head and the body of the pancreas (Fig. 2) showed that generally the uptake in the head of the pancreas was higher than in the body. In both the head and the body, the concentration was lower in the group of T1D patients.

The time between the diagnosis of T1D and the imaging ranged from 7 to 41 years. As demonstrated in Figure 3, the uptake of 111In-exendin in the pancreas appeared to be lower in patients with a longer history of T1D. (Pearson's correlation

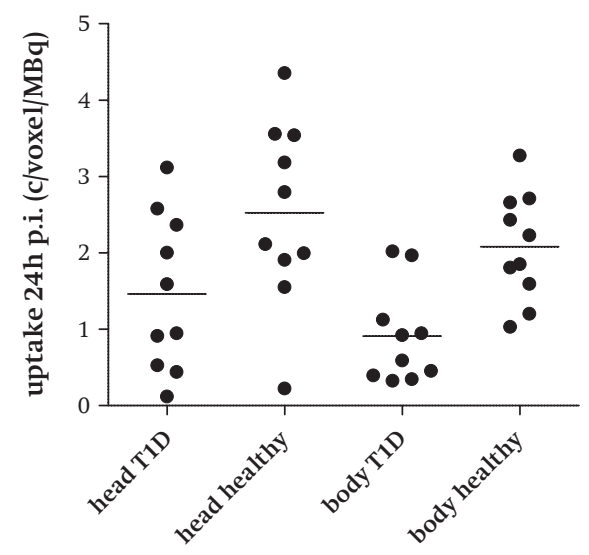

Figure 2 The activity concentration in the regions of interest drawn in the head (left) and body (right) of the pancreata of both T1D patients and healthy volunteers.

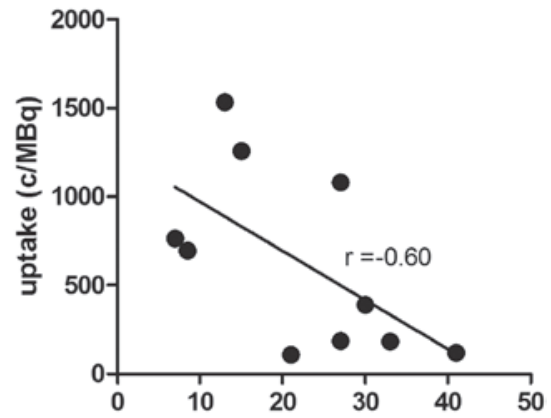

years since diagnosis

Figure 3 Uptake $24 \mathrm{~h}$ post injection versus the time that has passed since the diagnosis of type 1 diabetes.

coefficient $(\mathrm{r}=-0.60, \mathrm{P}=0.036)$. No relation was found between the age at onset of T1D and the uptake (Pearson $\mathrm{r}=-0.35, \mathrm{P}=0.16$ )

\section{Pancreas activity over time}

Of five T1D and four healthy volunteers images were acquired until $168 \mathrm{~h}$ post injection. These data were used to study the retention of ${ }^{111} \mathrm{In}$ in the pancreas. During 7 days, the amount of ${ }^{111}$ In in the pancreas, corrected for decay, remained stable (Fig 4)

\section{Activity in the kidneys}

The kidneys showed the highest retention of the radiotracer, even higher than the pancreas. Twenty-four hours post injection, the average kidney versus pancrea ratio in healthy volunteers was 29:1 (range 13:1 - 80:1) and in the T1D patients 68:1 (range 24:1 - 156:1). Since the reduction of activity in the kidney is faster than in the pancreas, the ratio was lower at later time points. The average kidney to pancreas ratio at $168 \mathrm{~h}$ post injection was 11:1 in T1D patients (range 5:1 - 17:1) and $6: 1$ in healthy volunteers (range 3:1-9:1)

\section{Uptake in other organs}

The planar images confirmed that no high uptake was seen in other organs than the kidneys and the pancreas. Organs with only slight (barely visible) uptake were the salivary glands and the upper gastro-intestinal tract. In contrast to preclinica imaging of rodents, no exendin uptake was observed in the lungs or the liver. 


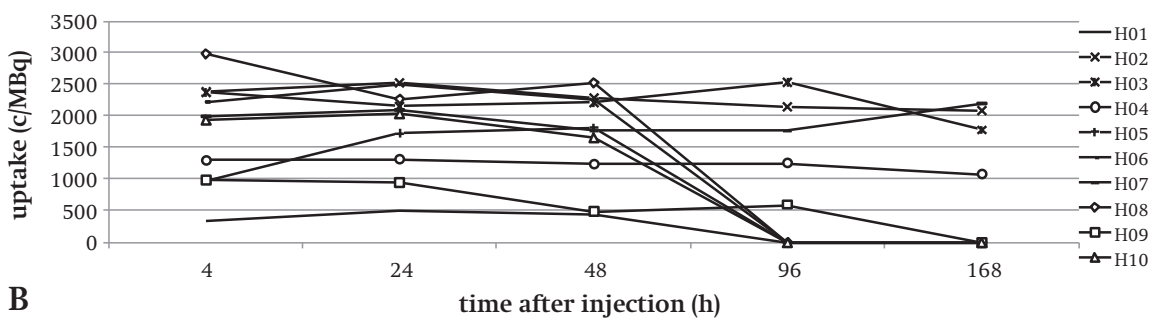

B

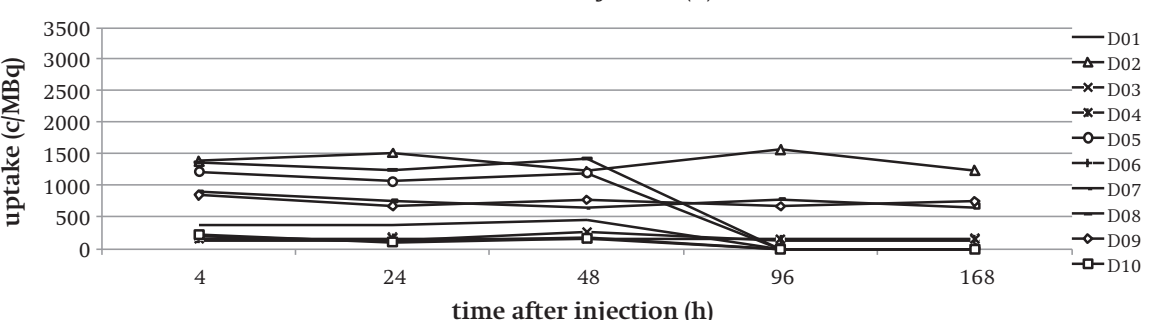

time after injection (h)

Figure 4 Activity in the pancreas at different time points after injection. corrected for decay. A) The results of the healthy volunteers. One volunteer did not have a SPECT at $128 \mathrm{~h}$ post injection. B) The results of the T1D patients.

\section{Discussion}

Here, we present the results of the first completed clinical study using radiolabeled exendin as an imaging biomarker for the BCM. Exendin has been identified as a promising imaging biomarker for the BCM for several reasons. Currently, no clinical imaging technology is able to resolve single islets of Langerhans in vivo which have a diameter of less than $250 \mu \mathrm{m}$ (13). Therefore, imaging of islets in vivo has to rely on "chemical resolution", i.e. a tracer molecule taken up with high specificity by the target cells so that the signal measured in a given organ will originate from the target cells (14). Indeed, for exendin such high specificity has been demonstrated in rodent models and GLP-1 receptor expression in the endocrine pancreas as compared to the exocrine pancreas appears to be more favourable in humans than in rats (10). In addition, the alpha cell mass has no influence on the pancreatic uptake of the radiotracer (11).

Currently, radionuclide imaging is the most sensitive method in biomedical imaging, able to detect picomolar concentrations of a tracer molecule. In case of radiolabeled exendin, the tracer molecule is actively internalized by the beta cells after binding to the receptor. The radiometal/chelator complex lysine-DTPA-111 In remains trapped in the lysosomes of the cells, an effect called "metabolic trapping", resulting in excellent target to background ratios as the non-internalized radiotracer clears rapidly from the background and is excreted via the kidneys (15).

When compared to imaging with ${ }^{11} \mathrm{C}-\mathrm{HTP}(9)$, our data demonstrate an inter-individual variation of the signal in the group of T1D patients as well as in healthy volunteers that exceeds the differences observed with HTP and resembles the inter-individual differences in BCM reported in the literature more closely (16) supporting the idea of a higher specificity of the exendin-based tracer for beta cells. As compared to DTBZ, the differences between the two groups were much more pronounced and uptake in several diabetes patients has dropped to very low values, close to the detection limit, which could not be observed with DTBZ (8).

The overlap between the group of T1D patients and healthy volunteers is larger in this study than observed with ${ }^{11} \mathrm{C}$-HTP. Although this overlap is for the major part caused by one patient with T1D with a extraordinarily high pancreatic uptake and one healthy volunteer with a very low uptake, these data are highly interesting a they support the hypothesis that in a considerable number of T1D patients, beta cell dysfunction may be responsible for the onset of the disease, a phase of the disease proceeding the actual loss of BCM (17). Interestingly, we found a lower uptake of ${ }^{111}$ In-exendin in subjects with a longer history of T1D, indicating that ${ }^{111}$ In-exendin uptake may reflect progressive beta cell loss over time. Indeed, Meier et al (16) demonstrated that beta cell apoptosis is an ongoing process during the course of T1D. Also, they demonstrated that the amount of insulin positive cells in the pancreas was about 2 times lower in patients with a longer history of T1D (4-23 $\mathrm{y}$ versus 33-67 y), while no relation between the number of beta cells and age/age at onset of disease was found. In addition, the presence of beta cells in the majority (at least $88 \%$ ) of the T1D patients in their study (16) could be demonstrated, an observation confirmed by others (18). These data are in agreement with the findings presented here.

Therefore, we believe that exendin imaging reflects pancreatic BCM an therefore represents an imaging technology that could be used to further elucidate the pathophysiology underlying the development and progress of T1D as well as T2D. Clinically, exendin imaging could be a valuable tool to identify individuals that may profit from treatment altering the course of the disease (for example by immunomodulation) or aiming at beta cell regeneration. Possibly, the combination of exendin imaging and HTP imaging might provide complementary information about changes in beta cell mass as compared to changes in total endocrine mass in the course of diabetes.

The exendin-based tracer described here is available as a GMP (good manufacturing practice) kit formulation allowing its use for human studies. Simple addition of ${ }^{111} \mathrm{InCl}_{3}$ to the vial containing the exendin-DTPA conjugate reveals the final product ready for injection after incubation at room temperature for 20 minutes. We have 
validated a protocol for quantitative SPECT imaging that can easily be implemented, making this technology available for human use in virtually any Nuclear Medicine department equipped with a SPECT/CT camera. We demonstrated that there is rapid uptake of the radiotracer into the pancreas and that this uptake remains stable over time, so that imaging can be performed in one day. Therefore, this technology has the potential to be used as an easy-to-use tool for clinical diabetes research (also suitable for efficient screening), not requiring a cyclotron facility (as is the case for DTBZ or HTP).

\section{Conclusion}

In conclusion, here we demonstrate the feasibility of exendin imaging in human for diabetes research. The data presented here (namely the significant difference between the pancreatic uptake of ${ }^{111}$ In-exendin in T1D patients and healthy volunteers, the inter-individual variation in uptake within the groups, the reduction of uptake during the course of the disease and residual uptake even in patients with long standing T1D) are in line with the available literature about BCM in T1D as well as with preclinical data. Therefore, quantitative exendin imaging appears to reflect human pancreatic BCM. This technology could represent a major assert on our way to better understand the pathophysiology of T1D (as well as T2D). The currently available easy-to-use kit formulation allows the ubiquitous utilization of this methodology for diabetes research. Validation of this promising tracer in clinical studies with T1D and T2D patients is under preparation.

\section{Acknowledgements}

We are most grateful the T1D patients and healthy volunteers who participated in this study.

The authors thank the technicians, physicians and especially the secretaria staff of the department of Radiology and Nuclear Medicine at the Radboud university medical center for their excellent assistance in the recruitment, planning, screening preparation of the ${ }^{111}$ In-exendin and imaging of the patients and volunteers. We very much acknowledge the physicians of the department of internal medicine (Radboud university medical center, Nijmegen, the Netherlands) for patient recruitment. We acknowledge W. Grootjans and M. Boss for their technica assistance in image analysis of the patient data and I. van der Kroon for her help in the writing process.

M.G. is the guarantor of this work and, as such, had full access to all the data in the study and takes responsibility for the integrity of the data and the accuracy of the data analysis.

\section{References}

1. Krogvold L, Edwin B, Buanes T, Ludvigsson J, Korsgren O, Hyoty H, et al. Pancreatic biopsy by minima Diabetologia. 2014;57(4):841-3. Epub 2014/01/17.

2. Tuomi T, Santoro N, Caprio S, Cai M, Weng J, Groop L. The many faces of diabetes: a disease with increasing heterogeneity. Lancet. 2014;383(9922):1084-94. Epub 2013/12/10.

3. Kahn SE, Cooper ME, Del Prato S. Pathophysiology and treatment of type 2 diabetes: perspectives on the past, present, and future. Lancet. 2014;383(9922):1068-83. Epub 2013/12/10.

4. Cleland SJ, Fisher BM, Colhoun HM, Sattar N, Petrie JR. Insulin resistance in type 1 diabetes: what is 'double diabetes' and what are the risks? Diabetologia. 2013:56(7):1462-70. Epub 2013/04/25.

5. Goke B. What are the Potential Benefits of Clinical beta-Cell Imaging in Diabetes Mellitus? Curren pharmaceutical design. 2010;16(14):1547-9.

6. Kahn SE. Quantifying beta-cells in health and disease: the past, the present, and the need. Diabetes care. 2013;36(1):4-5. Epub 2012/12/25.

7. Gotthardt M, Eizirik DL, Cnop M, Brom M. Beta cell imaging - a key tool in optimized diabetes prevention and treatment. Trends in endocrinology and metabolism: TEM. 2014;25(8):375-7. Epub 2014/04/15.

8. Freeby M, Ichise M, Harris PE. Vesicular monoamine transporter, type 2 (VMAT2) expression as it compares to insulin and pancreatic polypeptide in the head, body and tail of the human pancreas. Islets. 2012;4(6):393-7. Epub 2012/12/12.

9. Eriksson O, Espes D, Selvaraju RK, Jansson E, Antoni G, Sorensen J, et al. The Positron Emission Tomography ligand [11C]5-Hydroxy-Tryptophan can be used as a surrogate marker for the human endocrine pancreas. Diabetes. 2014. Epub 2014/05/23.

10. Brom M, Woliner-van der Weg W, Joosten L, Frielink C, Bouckenooghe T, Rijken P, et al Non-invasive quantification of the beta cell mass by SPECT with ${ }^{111}$ In-labelled exendin. Diabetologia 2014:57(5):950-9. Epub 2014/02/04.

1. Brom M, Joosten L, Frielink C, Boerman O, Gotthardt M. 111In-exendin uptake in the pancrea correlates with the beta cell mass and not with the alpha cell mass. Diabetes. 2014. Epub 2014/11/21

12. Report of the Expert Committee on the Diagnosis and Classification of Diabetes Mellitus. Diabetes care. 1997;20(7):1183-97. Epub 1997/07/01.

13. Hellman B. The frequency distribution of the number and volume of the islets Langerhans in man. Studies on non-diabetic adults. Acta Societatis Medicorum Upsaliensis. 1959;64:432-60. Epu 1959/01/01

14. Andralojc K, Srinivas M, Brom M, Joosten L, de Vries IJ, Eizirik DL, et al. Obstacles on the way to the clinical visualisation of beta cells: looking for the Aeneas of molecular imaging to navigate between Scylla and Charybdis. Diabetologia. 2012;55(5):1247-57. Epub 2012/02/24,

15. Brom M, Andralojc K, Oyen WJ, Boerman OC, Gotthardt M. Development of radiotracers for the determination of the beta-cell mass in vivo. Current pharmaceutical design. 2010;16(14):1561-7. Epub 2010/02/12

16. Meier JJ, Bhushan A, Butler AE, Rizza RA, Butler PC. Sustained beta cell apoptosis in patients wit long-standing type 1 diabetes: indirect evidence for islet regeneration? Diabetologia. 2005;48(11):22218. Epub 2005/10/06.

17. Pugliese A, Vendrame F, Reijonen H, Atkinson MA, Campbell-Thompson M, Burke GW. New insight o human type 1 diabetes biology: nPOD and nPOD-transplantation. Current diabetes reports. 2014;14(10):530. Epub 2014/08/22.

18. Keenan HA, Sun JK, Levine J, Doria A, Aiello LP, Eisenbarth G, et al. Residual insulin production and pancreatic ss-cell turnover after 50 years of diabetes: Joslin Medalist Study. Diabetes. 2010;59(11):284653. Epub 2010/08/12. 


\section{6}

A 3D-printed anatomical kidney and pancreas phantom for optimizing SPECT/CT acquisition and reconstruction settings in beta cell imaging using

${ }^{111}$ In-exendin

Wietske Woliner - van der Weg, Laura N. Deden, Antoi P.W. Meeuwis,

Maaike Koenrades, Laura H.C. Peeters, Henny Kuipers, Geert Jan Laanstra,

Martin Gotthardt, Cornelis H. Slump, Eric P. Visser 


\section{Abstract}

Quantitative single photon emission computed tomography (SPECT) is challenging in general. Imaging and quantification of the pancreatic beta cells with ${ }^{111} \mathrm{In}$ exendin puts even higher demands on image acquisition, reconstruction and quantification, due to high uptake in the kidneys versus low uptake in the pancreas Commonly used SPECT/computed tomography (CT) reconstruction protocols lead to several artefacts and effects of reconstruction software and settings on quantification remain uncertain. Therefore we designed a 3-dimensionally (3D)-printed phantom representing the pancreas and kidneys, to mimic the human situation in beta cel imaging and assess the effect of different acquisition and reconstruction settings and software on the quantification of the pancreas uptake.

Methods: Pancreas and kidneys, based on a patient's magnetic resonance image, were 3D-printed and inserted within the National Electrical Manufacturers Association (NEMA) NU2 image quality (IQ) phantom casing. The organs and background were filled with activities simulating a relatively high pancreatic ${ }^{111}$ In-exendin uptake (healthy), and low uptake (type 1 diabetes). Three different acquisition protocols were tested: 128 views with $40 \mathrm{~s} /$ view, 64 views with $80 \mathrm{~s}$ / view and 32 views with 160 s/view.

SPECT images were reconstructed using Siemens' Flash 3D and Hermes' Hybric Recon, with varying numbers of iterations and subsets, and with and without scatter attenuation and collimator corrections. Images were assessed on homogeneity, artefacts (both visually), and quantitative reliability (pancreas-to-kidney ratio).

Results: Phantom images showed similar artefacts as clinical images: over- and undershoot artefact in kidney area (all reconstructions with scatter correction) areas with a higher background intensity (most intense in Flash 3D between kidneys), and a spotted distribution in the pancreas (more with more iterations and subsets). All corrections were required to visualize the pancreas. Increased numbers of subsets and iterations improved the quantitative performance, but decreased homogeneity, both in the pancreas and background. Altogether, the Hybrid Recon reconstruction with 6 iterations and 16 subsets was selected for clinical use. Acquisition with 64 views resulted in most accurate quantification.

Conclusion: Use of the 3D-printed phantom elucidated how clinical images of ${ }^{111}$ In-exendin should be interpreted, and enabled selection of the best acquisition and reconstruction protocol for clinical use. It strongly contributed to enable quantification of the pancreatic ${ }^{111}$ In-exendin uptake in clinical images.

\section{Introduction}

The development of a non-invasive, imaging-based method for quantification of the beta cell mass could greatly enhance our understanding of the complex pathophysiology underlying the development of diabetes. Therefore, we have studied the use of pancreatic uptake of ${ }^{111}$ In-exendin as an imaging biomarker for the beta cell mass preclinically, and recently also in type 1 diabetes (T1D) patients and healthy humans (1).

To improve quantification of pancreatic ${ }^{111}$ In-exendin uptake in this clinical study, a phantom mimicking the human situation was required. Quantitative single photon emission computed tomography (SPECT) is challenging in genera (2), and imaging and quantification of the pancreatic beta cells with ${ }^{111}$ In-exendin is even more challenging, due to the high uptake in the kidneys versus the low uptake in the pancreas (1).

The pancreas, and especially the tail of the pancreas, is located close to the kidneys. Compared to the pancreas, the kidneys have a very high uptake with an average pancreas-to-kidney ratio of about 1:30 in healthy humans and about 1:70 in diabetes patients (1). Initially human images were reconstructed with the SPECT/computed tomography (CT) protocol that we generally use in the clinic Flash 3D, a SPECT 3D ordered subset expectation maximization (OSEM) algorithm within Syngo (Siemens Inc, Munich, Germany), with 6 iterations and 16 subsets. After imaging the first humans, the data were also reconstructed with Hybrid Recon oncology (Hermes Medical Solutions, Stockholm, Sweden) and ReSPECT (Scivis Germany). The initial settings for these reconstructions are given in table 1 In these images we observed features that could probably be attributed to artefacts: a low count rim surrounding the kidneys in the Flash 3D reconstructions and less pronounced in the Hybrid Recon reconstructions and a high intensity focus in the vertebral area in the ReSPECT reconstructions. These features are shown in Table 1.

These findings were not surprising, because these reconstruction protocols were developed to visualize normal uptake and non-extreme contrast, for example for highly sensitive lesion detection (e.g. metastases), and not to quantify low uptake in a specific organ, in this case the pancreas. In images of humans, the origin of artefacts, their influence on quantification, and sometimes even the presence of artefacts cannot reliably be investigated because the true distribution of the radiotracer remains unknown. Also, details about the working of commercially available reconstruction software are not always available to the user; partially the software acts like a black box. Therefore, the use of a phantom is of major importance for recognizing and understanding the origin of reconstruction artefacts, and in our case to enable reliable quantification in beta cell imaging. Normally, 
Table 1 Initial reconstruction settings and findings

\begin{tabular}{|c|c|c|c|}
\hline & Flash 3D & Hybrid Recon & ReSPECT \\
\hline Version & $\begin{array}{l}\text { Siemens Syngo } \\
\text { MI.SPECT } \\
\text { application E.soft } \\
2009 \text { a, 8.1.15.7 } \\
\text { service pack } 2\end{array}$ & $\begin{array}{l}\text { HERMES P5 GOLD } \\
4.6 \mathrm{~A}\end{array}$ & ReSPECT V2.5 \\
\hline $\begin{array}{l}\text { \# iterations - } \\
\text { \# subsets }\end{array}$ & $6-16$ & $4-16$ & $6-32$ \\
\hline $\begin{array}{l}\text { Attenuation } \\
\text { correction }\end{array}$ & CT-based & CT-based & $\begin{array}{l}\text { Based on body } \\
\text { contour } \mu=0.13 \mathrm{~cm}^{-1}\end{array}$ \\
\hline Scatter correction & $\begin{array}{l}\text { Tripple energy } \\
\text { window }\end{array}$ & Monte Carlo based & None \\
\hline Filtering & Gauss $0.84 \mathrm{~cm}$ & Gauss $0.96 \mathrm{~cm}$ & $\begin{array}{l}\text { Background } \\
\text { subtraction }\end{array}$ \\
\hline $\begin{array}{l}\text { Most obvious } \\
\text { artefacts in the first } \\
\text { patient images }\end{array}$ & $\begin{array}{l}\text { Large low-intensity } \\
\text { rim around kidneys }\end{array}$ & $\begin{array}{l}\text { Small low intensity } \\
\text { rim around the } \\
\text { kidneys, a little } \\
\text { increased intensity } \\
\text { in the vertebrae area }\end{array}$ & $\begin{array}{l}\text { Hot 'spot' in the } \\
\text { vertebrae area }\end{array}$ \\
\hline
\end{tabular}

phantoms in nuclear medicine consist of simple geometrical shapes such as spheres cylinders, rods, etcetera, however, these are less suitable to mimic the ${ }^{111} \mathrm{In}$-exendin distribution in the pancreas and kidneys in humans. Therefore in this study an anatomical phantom was required.

In the last few years, three-dimensional (3D)-printing has become an established method for fast and affordable production of advanced and customized phantoms (3-5) Within this context, we employed this technique to create an anatomical phantom containing a pancreas, two kidneys and a background region for optimizing the SPECT/CT-reconstructions to allow quantification of the pancreatic uptake of ${ }^{111}$ In-exendin in the clinical study (1).

The primary objective of this work was to mimic human ${ }^{111}$ In-exendin imaging with this custom made anatomical phantom to clarify how the human images should be interpreted (e.g. whether artefacts play a role). In addition, the objective was to select and optimize the acquisition and reconstruction protocols for beta cell quantification.

We designed and printed 3D anatomical inserts representing the pancreas and kidneys that can be placed within the casing of the National Electrical Manufacturers Association (NEMA) NU2 image quality (IQ) body phantom (6) after removing the standard spheres and cylinder inserts.

This anatomical phantom was filled with known activity concentrations to simulate SPECT imaging of T1D patients (low pancreatic uptake) and healthy humans (somewhat higher pancreatic uptake). Software of two vendors and different reconstruction settings were tested to find the optimal software and settings. After evaluation, the optimal reconstruction protocol was applied to human data of the clinical study. Additionally, different acquisitions protocols were evaluated.

\section{Materials and Methods}

\section{Phantom development}

The kidney and pancreas organ models were based on an MRI dataset of a male patient of $87 \mathrm{~kg}$. Fourty sequential T2 weighted HASTE images were acquired with a Magnetom TrioTim 3.0 Tesla MRI scanner (Siemens Medical Solutions) as part of another clinical study. The volumes and distances between the organs measured in the MRI images were compared to CT images of humans imaged with ${ }^{111} \mathrm{In}$-exendin, to make sure the anatomy was representative for our study population.

Images were processed in Mimics v.14.0 (Materialise HQ, Leuven, Belgium). 3D models of the kidneys and the pancreas were created from segmentations that were performed on the axial images. The models were smoothed with a first order Laplacian (70 iterations, smooth factor 0.9) and exported as 3D triangular surface meshes (STL-files). Using the open source software Meshlab v.1.3.2 (http:|/meshlab. sourceforge.net/) the surfaces were further processed to create a realistic anatomical model; abnormal bulges and sharp edges were removed or smoothed and holes were filled. The solid bodies were transformed to hollow organ models with a wall thickness of $4 \mathrm{~mm}$ with Meshlab and SolidWorks (v. 2012, Dassault Systèmes SolidWorks Corp., Waltham, Massachusetts, USA). In the design, flanges were added for fixation of the organs. Then, the organ models were 3D-printed with an Objet Eden $250^{\mathrm{TM}}$ printer (Stratasys, Eden Prairie, MN, USA) based on ultra-thinlayer Polyjet ${ }^{\mathrm{TM}}$ technology. Specifications include a layer thickness of $16 \mu \mathrm{m}$, tray size of $260 \times 260 \times 200 \mathrm{~mm}$ and accuracy of $100 \mu \mathrm{m}$. The material used for printing was the transparent standard plastic VeroClear RGD810, with a polymerized density of 1.18-1.19 g/ $/ \mathrm{cm}^{3}$. The non-soluble support material was taken out by hand. 
To enable filling, holes were drilled in the organs. These holes were closed with nylon screws sealed with rubber O-rings. The printed organs were placed within the NEMA casing and fixated with screw threads in the original screw holes in the lid of the NEMA casing.

The volumes of the pancreas and kidney inserts were measured by weighting the organs empty and filled with water. The volume of the background in the NEMA casing was measured by filling it with water with a graduated cylinder.

\section{Reconstruction software}

Reconstruction software of Siemens, Syngo Flash 3D, and Hermes, Hybrid Recon, were applied. The originally used ReSPECT software (V2.5) was excluded for quantification of the ${ }^{111}$ In-exendin uptake, because CT-based attenuation correction was not possible and the intensity in the remainder of the body was too low for reliable determination of the body contour. Reconstruction settings are given in the experiment descriptions.

\section{Phantom experiment 1 - high pancreas activity \\ (simulating healthy individuals)}

Activity concentrations for the pancreas, kidneys and background in the phantom were based on an estimation of the uptake of ${ }^{111}$ In-exendin in Flash3D reconstructions of the first three healthy volunteers (thus with a relatively high pancreas uptake) from the clinical study (1). The intensity in the pancreas and kidneys of these humans was retrieved by delineating the pancreas and kidneys and a background volume using the Inveon Research Workplace (IRW) software (Siemens Inc, Munich, Germany). The corresponding amount of ${ }^{111}$ In activity to be inserted in the phantom compartments was calculated by multiplying these intensities with a calibration factor $(1.19 \mathrm{~Bq} /$ count). This factor was determined from SPECT imaging of a cylindrical phantom with the same acquisition and reconstruction settings as the human SPECTs. To avoid sticking of ${ }^{111}$ In to the phantom wall, $0.05 \%$ Tween was added to the solution. The pancreas-to-kidney activity ratio was 1 : 57 , the pancreas-to-background ratio 1: 0.005 . Table 2 provides the volumes of the compartments and the amounts of ${ }^{111}$ In activity within the compartments.

SPECT images were acquired using an integrated SPECT/CT scanner (Symbia T16, Siemens Healthcare, Molecular Imaging, Hoffman Estates, IL, USA) equipped with parallel-hole medium-energy collimators. All subjects were scanned using fixed radius of rotation $(25 \mathrm{~cm})$ and a fixed bed height $(13 \mathrm{~cm})$. We obtained 128 views in step-and-shoot mode (64 views per camera head) with an acquisition time of $40 \mathrm{~s}$ per view. Symmetric 15\% energy windows over the 172 and the $247 \mathrm{keV}$ photopeaks were used, with additional $7 \%$ lower and upper scatter windows. These are the same settings as previously used in the clinical study (1)
To determine the optimal amount of iterations and subsets for ${ }^{111}$ In-exendin different numbers of iterations and subsets were applied; 6/16 (6 iterations and 16 subsets), 12/16, 12/32, 18/16, 18/32, 24/32, 30/32 and 32/32 for Hybrid Recon and 3/16, 6/16, 6/62, 12/16, 12/32, 18/16, 18/32, 24/16, 24/32, 30/16 and 30/32 for Flash 3D. The analysis of the results is performed as described in subsection 'Image analysis'.

\section{Phantom experiment 2 - low pancreas activity}

\section{(simulating T1D patients)}

In the second experiment, phantom filling (see table 2) was based on measurement in T1D patients (relatively low pancreatic uptake compared to healthy humans) of the clinical study (1). The pancreas-to-kidney activity ratio was 1: 108, the pancreasto-background ratio 1: 0.01

Three different acquisitions were performed to test the effect of decreasing the number of views and increasing the scan time per view: (I) 128 views with 40 s/view, (II) 64 views with 80 s/view and (III) 32 views with 160 s/view.

The first experiment already confirmed that Hybrid Recon led to more representative activity ratios, therefore only Hybrid Recon was used for reconstruction of the data in this experiment

In this experiment, not only OSEM 3D, but also maximum a posteriori (MAP) reconstructions with a smoothing prior $\left(\mathrm{MAP}_{\text {smothing }}\right)$ and with a median root prior $\left(\mathrm{MAP}_{\mathrm{MRP}}\right)$ were made. With the smoothing prior attempting smoothing of the image including boundaries, it is hypothesized that the pancreas activity is more homogeneously distributed in these images. The MRP preserves edges and hotspots more than the smoothing prior, but also assumes a locally monotonic image (7). The $\mathrm{MAP}_{\text {smoothing }} \mathrm{MAP}_{\text {MRP }}$ and OSEM 3D images were reconstructed with three sets of iterations and subsets: 6/16, 12/16 and 18/32.

After analyzing these reconstructions (see section 'Image analysis'), we selected OSEM 3D and MAP ${ }_{\text {MRP }}$ reconstructions with 6 iterations and 16 subsets for determination of the effect of the different acquisition settings and of severa correction settings (scatter correction (SC), attenuation correction (AC), and collimator correction (CC)) in the reconstruction protocol. The effect of the corrections was tested by switching off the corrections consecutively.

\section{Image analysis}

To decide which reconstruction software and settings and which acquisition protocol lead to the best images for quantification of the ${ }^{111}$ In-exendin uptake in the pancreas, all images were visually assessed and intensities of the differen compartments were determined. 
Table 2 Phantom and filling characteristics

\begin{tabular}{|c|c|c|c|c|c|c|}
\hline & & & Pancreas & $\begin{array}{l}\text { Left } \\
\text { kidney }\end{array}$ & $\begin{array}{l}\text { Right } \\
\text { kidney }\end{array}$ & Background \\
\hline & Volume (mL) & & 82.3 & 206 & 222 & 9260 \\
\hline \multirow[t]{2}{*}{ Experiment 1} & $\begin{array}{l}\text { Concentration } \\
{ }^{111} \mathrm{In} \text { at scan time } \\
(\mathrm{MBq} / \mathrm{mL})\end{array}$ & & $3.94^{*} 10^{-3}$ & 0.226 & 0.226 & $2.11^{*} 10^{-5}$ \\
\hline & $\begin{array}{l}\text { Total activity } \\
{ }^{111} \text { In at scan time } \\
\text { (MBq) }\end{array}$ & & 0.32 & 46.56 & 50.17 & 0.20 \\
\hline \multirow[t]{6}{*}{ Experiment 2} & \multirow{3}{*}{$\begin{array}{l}\text { Concentration } \\
\text { 111 In at scan time } \\
(\mathrm{MBq} / \mathrm{mL})\end{array}$} & Acq 1 & $1.93^{*} 10^{-3}$ & 0.209 & 0.209 & $2.10^{*} 10^{-5}$ \\
\hline & & Acq 2 & $1.88^{*} 10^{-3}$ & 0.204 & 0.204 & $2.05^{*} 10^{-5}$ \\
\hline & & Acq 3 & $1.87^{*} 10^{-3}$ & 0.202 & 0.202 & $2.03^{*} 10^{-5}$ \\
\hline & \multirow{3}{*}{$\begin{array}{l}\text { Total activity } \\
{ }^{111} \text { In at scan time } \\
\text { (MBq) }\end{array}$} & Acq 1 & 0.16 & 43.14 & 46.49 & 0.19 \\
\hline & & Acq 2 & 0.15 & 42.02 & 45.29 & 0.19 \\
\hline & & Acq 3 & 0.15 & 41.14 & 46.89 & 0.19 \\
\hline
\end{tabular}

Acq = acquisition

Visual assessment focussed on homogeneity within the pancreas and kidneys, on artefacts, and the position of the pancreas in the CT images compared to the position in the SPECT images.

Using IRW, pancreas and kidney volumes of interest (VOI) were delineated and for the background a box-shaped VOI was defined. Each VOI was used for retrieving the average intensity as a measure for the inserted activity in these compartment. The measured intensities were compared to real activities in the phantom. Also, pancreas-to-background and pancreas-to-kidney intensity ratios were calculated and compared to the real activity concentration ratios.

\section{Verification of phantom-based optimization on human images}

As a final test, the optimized reconstruction protocol was applied to data of the clinical study (1). Reconstructions were performed with Hermes Hybrid Recon, with 6 iterations and 16 subsets (see table 3) and visually compared to the initial reconstruction with Flash 3D and the phantom images.

\section{Results}

SPECT imaging of the 3D printed phantom led to images that were, in terms of visualization of the pancreas and the kidneys and artefacts, comparable with human images (see figure 1).

\section{Homogeneity and intensity within the organs}

Both with Hybrid Recon and Flash 3D, increasing the number of iterations or subsets led to noisier images, which results in more spots in the pancreas (see figure 2 for Hybrid Recon, Flash 3D gives similar results). In humans, the spots in the pancreas could easily be interpreted as inhomogeneous uptake of ${ }^{111}$ In-exendin.

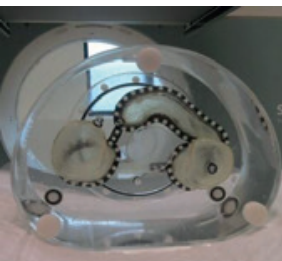

A

Phantom

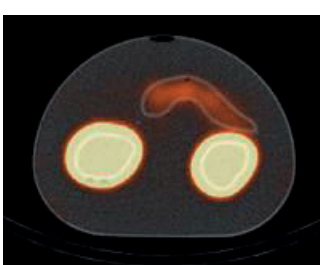

B

Phantom

Hybrid Recon OSEM

6 iterations 16 subsets

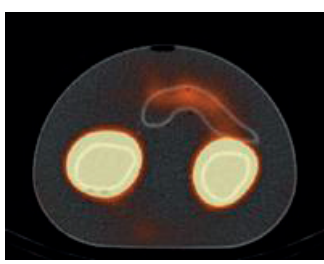

D

Phantom

Flash $3 \mathrm{D}, 6$ iterations

and 16 subsets

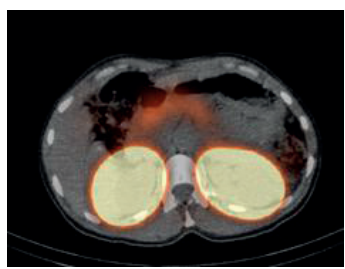

C

Patient reconstructed Hybrid OSEM Recon 6 iterations 16 subsets

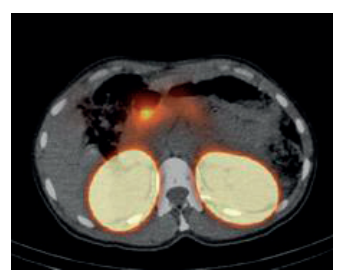

E

Healthy subjec Flash 3D, 6 iteration and 16 subsets
Figure 1 A) Photograph of the 3D-printed pancreas and kidney compartments in the NEMA-NU2 phantom casing Reconstructions of the phantom SPECT/CT from experiment 1 (B and D) and a healthy human (C and E). Figure B and E show reconstructions made with Hybrid Recon OSEM with attenuation, collimator and scatter correction, 6 iterations and 16 subset. Figure D and E show Flash 3D reconstructions with 6 iterations and 16 subsets. Window settings are the same in all images 


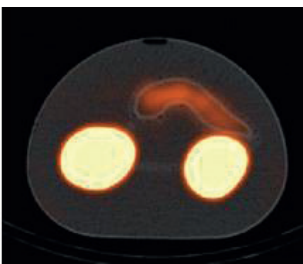

A

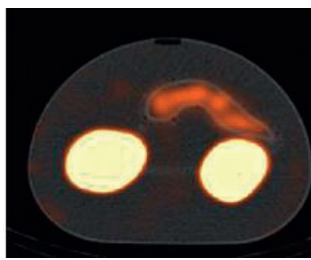

B

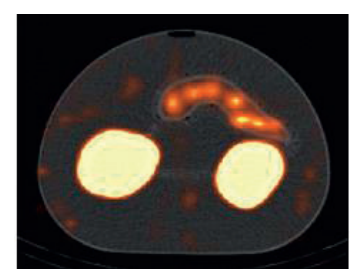

C
Figure 2 The effect of increasing the number of iterations: Figure A) was made with 6 iterations and 16 subsets, B) with 12 iterations and 32 subsets and C) with 32 iterations and 32 subsets. All images were reconstructed with Hybrid Recon OSEM with attenuation, collimator and scatter correction. Window settings are the same in all images.

Furthermore, increasing the number of iterations and subsets increases the average intensity of the pancreas and more strongly influences the pancreas compared to the kidneys. For example, comparing 6/16 and 30/32 the relative difference is approximately $40 \%$ in the pancreas (Flash 3D 43\%, Hybrid Recon 35\%) and 5\% in the kidneys (Flash 3D 7\%, Hybrid Recon 3\%).

In Flash 3D reconstructions, the intensity in the tail and the edge of the head (close to the kidney) is lower compared to the other parts of the pancreas. Also, it seems that the pancreas position on SPECT is more towards anterior compared to CT (Figure 1D). In Hybrid Recon the position of the pancreas is similar in SPECT and $\mathrm{CT}$ and the intensity is more homogeneous across the entire pancreas. For a high number of iterations (18 iterations, 32 subsets) in Hybrid Recon, the intensity distribution in the pancreas is more homogeneous with the MAP reconstructions compared to the OSEM reconstruction.

In both Flash 3D and Hybrid Recon the intensity in the kidneys is not homogeneous (see 'Artefacts' and Figure 3) and the background activity was low and inhomogeneous. The average intensity in the background was similar in all Flash 3D and Hybrid Recon reconstructions (Flash 3D 16 ( \pm 1.4$)$ counts/ voxel, Hybrid Recon 1.8 ( \pm 0.26$)$ counts/ voxel).

The effect of applying corrections was tested with Hybrid Recon OSEM and MAP with 6 iterations and 16 subsets. Notably, applying the SC on top of the AC and CC influences the low intensity areas (pancreas and background) more than the high intensity areas (kidneys). Without the use of all corrections the pancreas was not visible in images of the second experiment (simulating T1D pancreas activity), so even with only the $\mathrm{CC}$ and $\mathrm{AC}$ on, the pancreas cannot be distinguished from the background.

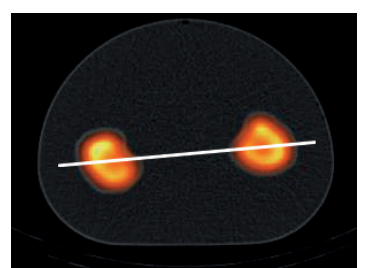

A

Hermes OSEM 3D 6 it 16 subs

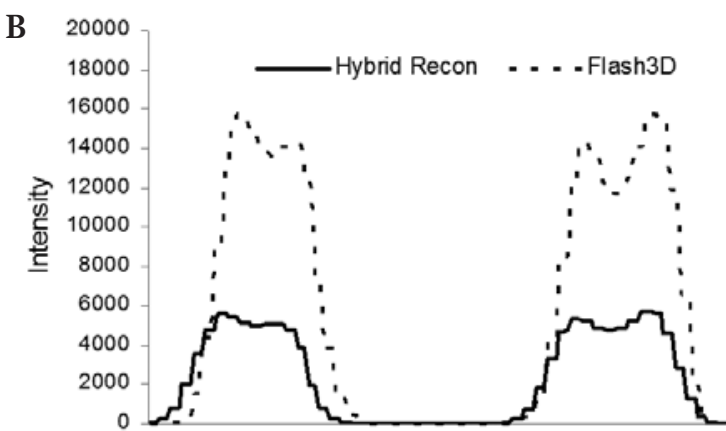

Position
C

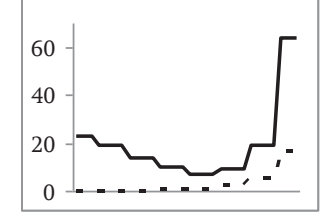

Figure 3 Figure A) shows the Hybrid Recon OSEM reconstruction with attenuation, collimator and scatter correction, 6 iterations and 16 subsets. The line in A) is used to construct the profile shown in B). B) shows also the line profile at the same position of the Flash 3D reconstruction with similar settings. A close up of the graph in B) is shown in C) This shows why Flash 3D has a cold rim around the kidneys, compared to the Hybrid Recon reconstructions. The Flash 3D line drops to zero.

\section{Artefacts}

In all Flash 3D and Hybrid Recon reconstructions, there seems to be a high intensity rim inside the kidneys, as if there were a cortex in the phantom with a higher activity (see figure 3A), all images also show a low-intensity shell around the kidneys. This ring artefact with under- and overshoot appears like the Gibbs phenomenon. In the Flash 3D images the low-intensity ring is more pronounced than in the Hybrid Recon reconstructions.

When SC is turned off, the low-intensity rim disappears and the activity in the kidneys is more homogeneous.

Both Hybrid Recon and Flash 3D showed an area in the background in which the intensity is higher than the average background intensity. For Flash 3D this area is located between the kidneys, in Hybrid Recon this area is less intense and located more to the back, centrally posterior of the kidneys (see figure 4). With $\mathrm{MAP}_{\text {s }}$ this artefact was least pronounced. 


\section{Activity concentration ratios}

Figure 5 shows the pancreas-to-kidney intensity ratio for reconstructions with Hybrid Recon and Flash 3D with different numbers of iterations and subsets. For both reconstruction programmes, the pancreas-to-kidney ratio increase with increasing number of iterations times the number of subsets. The ratio of the Hybrid Recon reconstruction is much closer to the real ratio than the Flash 3D ratio. The OSEM reconstruction leads to more reliable ratios than the MAP reconstructions. For 12 iterations with 16 subsets, the pancreas to kidney ratio is 1:122 with OSEM, 1:137 for $\mathrm{MAP}_{\text {MRP }}$ and 1:172 for $\mathrm{MAP}_{\text {smoothing }}$, while the real ratio was $1: 108$.

The average pancreas-to- background ratios from respectively the Flash 3D and the Hybrid Recon OSEM reconstructions were 1:0.21 and 1:0.037. The actual ratio was 1: 0.005. Within Hybrid Recon, the OSEM reconstructions (compared to the MAP reconstructions) lead to ratios that were closest to the actual ratio.

\section{Verification of results on human data}

After comparing the results of the different reconstruction protocols, Hybrid Recon with the OSEM reconstruction with 6 iterations, 16 subsets, SC, AC and CC, was selected for reconstruction of the human data, analysed for our clinical trial (1) (Figure 1C). Small differences can be observed between this Hybrid Recon reconstruction and the initial Flash 3D reconstruction (Figure 1E). In Hybrid Recon the intensity within the background and pancreas is more homogeneous compared to Flash 3D, and a difference in shape of the pancreas was observed. The human SPECT images are comparable to the phantom images reconstructed with the optimal Hybrid Recon settings (Figure 1B) and initial Flash 3D settings (Figure 1D).

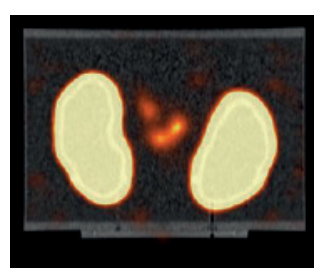

Figure 4 A hotspot with the same intensity as the pancreas is located between the kidneys in the Hybrid Recon reconstructions. This image was reconstructed with OSEM, with 12 iterations and 32 subset, attenuation, scatter and collimator correction.
A

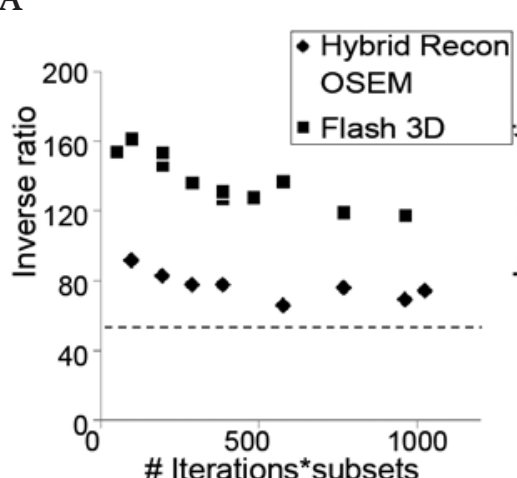

200

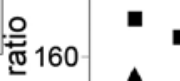

¿ 80

0
0
\# Iterations*
$*$

Figure 5 The effect of the number of iterations, times the number of subsets and the use of different reconstruction methods on the inverse pancreas-to-kidney (i.e. kidney-topancreas) intensity ratio. The actual inverse activity ratio, as inserted in the phantom is depicted with the striped line. A) Shows results of the first experiment. B) Shows results of the second experiment.

\section{Effect of different acquisition protocols}

Table 3 shows the actual and the measured pancreas-to-kidney and pancreas-tobackground ratios for the three acquisitions in the second experiment. As can be seen in this table, for the OSEM reconstructions with 6 iterations and 16 subsets, the pancreas-to-kidney ratio was similar for the different acquisition protocols (1:138, 1:143 and 1:143). With the $\mathrm{MAP}_{\mathrm{MRP}}$ reconstruction, the ratio is the lowest (and closest to the actual ratio) in the first acquisition (128 views, 40s/view), namely 1:149.

The pancreas-to-background ratio varied among the different acquisitions and protocols (see table 3).

The hotspot between the kidneys became less apparent when decreasing the number of views. 


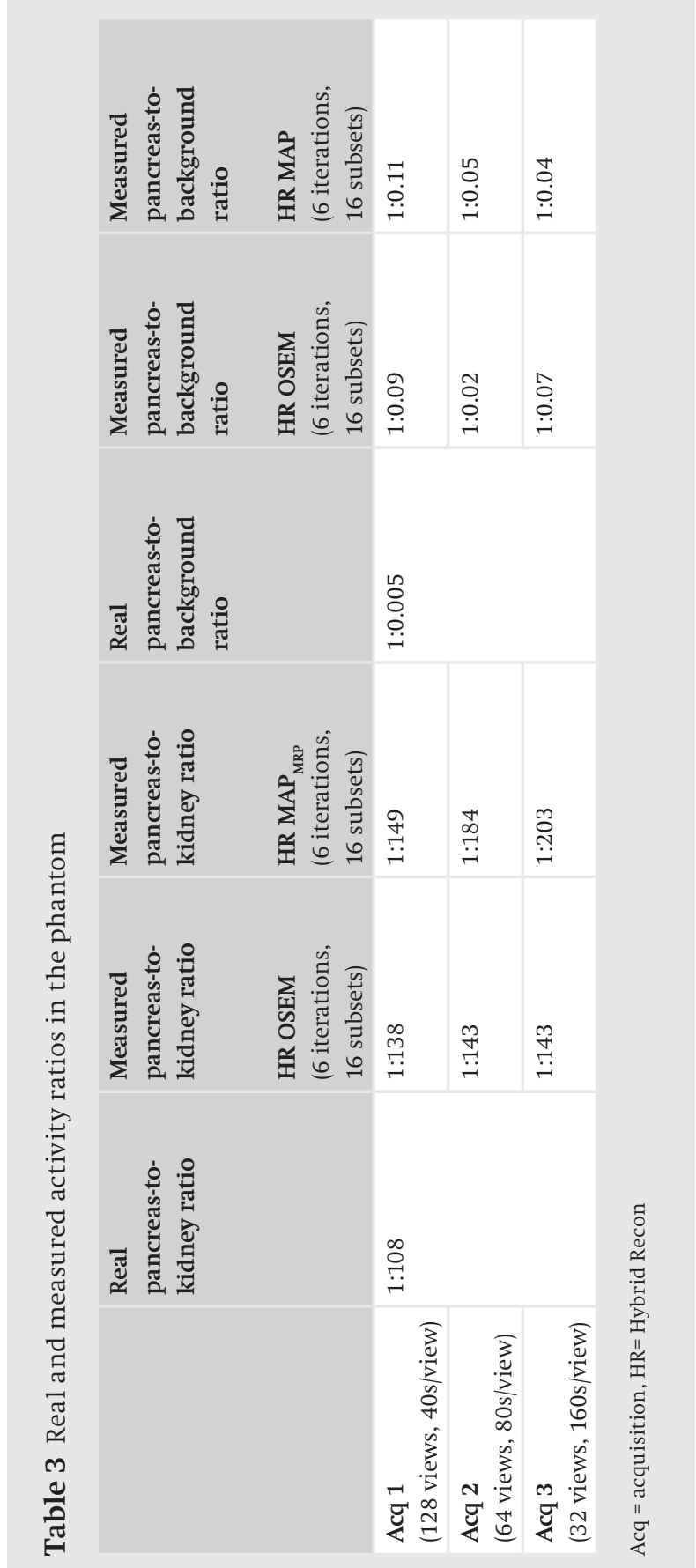

\section{Discussion}

We have developed a phantom that mimics the human situation for ${ }^{111}$ In-exendin maging, by 3D-printing pancreas and kidney compartments for insertion into the NEMA-NU2 casing. The phantom was used for optimization of SPECT reconstruction and acquisition protocols. Non-anatomical phantoms could never mimic the actual ${ }^{111}$ In-exendin images of the pancreas and kidneys. Our 3D-printed phantom, however, led indeed to images, including artefacts, that appear similar to human ${ }^{11}$ In-exendin images.

The inserted activity in each compartment of the phantom was homogeneously distributed, and therefore in the reconstructed images the measured intensity should also be homogeneous. Lower numbers of iterations and subsets gave a more homogeneous intensity, especially in the pancreas. As a result of the incorporated smoothing or median root priors, the images from MAP reconstructions were smoother and more homogeneous within the compartments than the OSEM mages. Nevertheless, in human these MAP reconstructions could only be used with a lot of caution. In some human, the true spatial distribution of activity could be influenced by a higher glucagon-like peptide-1 (GLP-1) receptor expression in certain areas of the pancreas, e.g. in the case of an insulinoma. The use of a prior leading to smoothing, could reduce the visibility of such regions.

However the pancreas-to-kidney ratio improved by increasing the number of iterations and subsets, it also led to increased inhomogeneity in the pancreas, which does not correspond to the actual activity distribution.

Therefore an OSEM reconstruction with a relatively low number of iterations and subsets was chosen for the human reconstructions.

It seems that SC in both Flash 3D and Hybrid Recon, results in the Gibbs-like artefact. $\mathrm{SC}$ influences the intensity measured in the kidneys more strongly than in the pancreas. This could also explain why the actual kidney-to-pancreas ratio could not be reached with the used protocols. Visually on the SPECT image, the Gibbs-like artefact seems to be more pronounced in Flash 3D (because of a ring of voxels with value 0 ), however, quantitatively as demonstrated by the line profile (see Figure $4 \mathrm{~B}$ and C), the difference between Flash 3D and Hybrid Recon was less pronounced.

It appears that the position of the pancreas is more anterior in the Flash 3D reconstructions (figure $2 \mathrm{C}$ ). This might also be caused by the Gibbs-like artefact that 'absorbs' a part of the counts in the pancreas. Therefore it seems that the pancreas is visualized more anterior compared to the images reconstructed with Hybrid Recon. Because of the favourable visualization of the pancreas, Hybrid Hybrid Recon was chosen for the human reconstructions. 
It seems that overcorrection for scatter in both Flash 3D and Hybrid Recon, results in the Gibbs-like artefact. The SC influences the kidneys more strongly than the pancreas. This also explains why the actual kidney-to-pancreas ratio could not be reached with the used protocols. Visually, the Gibbs-like artefact seems to be more pronounced in Flash 3D (because of a ring of voxels with value 0), however, quantitatively, the difference between Flash 3D and Hybrid Recon was less pronounced (see Figure 3B).

It appears that the position of the pancreas is more anterior in the Flash 3D reconstructions (figure 1D). This might also be caused by the Gibbs-like artefact that 'absorbs' a part of the counts in the pancreas. Therefore it seems that the pancreas is visualized more anterior compared to the images reconstructed with Hybrid Recon. Because of the favourable visualization of the pancreas, Hybrid Hybrid Recon was chosen for the patient reconstructions.

SC, CC and AC showed to be necessary for beta cell imaging with ${ }^{111}$ In-exendin. So finally Hybrid Recon with an OSEM reconstruction with 6 iterations, 16 subsets, SC, CC and AC was used for the clinical study.

Comparing the original reconstruction with Flash 3D and the final reconstruction with Hybrid Recon shows only small differences. Since there were large differences between the tested protocols, we conclude that our original Flash 3D protocol comes very close to the now chosen optimal protocol with Hybrid Recon.

For future ${ }^{111}$ In-exendin imaging with SPECT, a different acquisition protoco should be used. With reducing the number of views to 64 , and increasing the time per view to $80 \mathrm{~s} /$ view, the total acquisition time remains the same, while the resulting image is more representative for the real situation.

\section{Conclusion}

3D-printing of a phantom that mimics the human pancreas and kidneys showed to be useful for optimization of ${ }^{111}$ In-exendin imaging. Phantom experiment enabled optimization of the reconstruction protocol and provided advice for future acquisitions in clinical patient studies. This work contributed to clarify the relation between SPECT ${ }^{11}$ In-exendin images and the actual situation in the patient. So, the use of this custom-made phantom provided essential information for beta cell quantification.

\section{References}

1. Brom M, Woliner-van der Weg W, Joosten L, Frielink C, Bouckenooghe T, Rijken P, et al. Non-invasive quantification of the beta cell mass by SPECT with ${ }^{111}$ In 9. Epub 2014/02/04.

2. Ritt P, Vija H, Hornegger J, Kuwert T. Absolute quantification in SPECT. European journal of nucleat medicine and molecular imaging. 2011;38 Suppl 1:S69-77. Epub 2011/04/13.

3. Gear JI, Long C, Rushforth D, Chittenden SJ, Cummings C, Flux GD. Development of patient-specific molecular iman Driscoll B. Keller $\mathrm{H}$. Coolens $C$. Development of a dynamic flow imaging phantom for dynamic contrast-enhanced CT. Medical physics. 2011;38(8):4866-80. Epub 2011/09/21.

5. Miller BW, Moore JW Gehm ME Furenlid LR, Barrett HH. Novel Applications of Rapid Prototyping in Gam BW Mora $\mathrm{X}$. G I

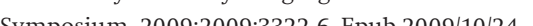

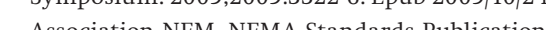

Association NEM. NEMA Standards Publication NU 2-2007. Performance Measurements of Positron Kangasmaa T, Sohlberg A, Kuikka JT. Reduction of collimator correction artefacts with bayesia
reconstruction in spect International journal of molecular imaging 2011:2011:630813. Epub 2011/04/15 
Integration of micro- and macro-dosimetry for calculation of radiation doses to the islets of Langerhans due to imaging with radiolabeled exendin

Inge van der Kroon, Wietske Woliner - van der Weg, Mark W. Konijnenberg, Eric P. Visser, Martin Gotthardt 


\section{Abstract}

The use of radiotracers for quantification of the insulin producing beta cells located in the islets of Langerhans in the pancreas, leads to concern about radiation induced damage to the islets. Estimation of the radiation effect requires a method for calculation of the radiation dose to these islets. Commonly used macro-dosimetry methods like OLINDA/EXM cannot be applied directly since the model included in this software do not include the islets of Langerhans as a separate compartment. Therefore a model was developed to calculate the islet radiation dose due to use of ${ }^{111} \mathrm{In}$-exendin and ${ }^{68} \mathrm{Ga}$-exendin, with a variable input and used for radiation dose calculations in several examples. As input for the model not only preclinical, but also clinical data was used.

Methods: The model developed includes the radiation dose to the islets due to (i) uptake of the radiotracer in the kidneys, due to uptake in (ii) surrounding islets and exocrine tissue in the pancreas and the (iii) islet self-dose. Activity in the remainder of the body is low and can therefore be neglected. The islet doses due to surrounding pancreas tissue and the kidneys is S-value based and for the self-dose of the islets Monte Carlo simulations were performed for a small sphere model (diameter 50-400 $\mu \mathrm{m}$ ). For calculation of the residence times both clinical and preclinical data were used. Five examples (including variations in gender, isle size, \% islets in the pancreas and kidney uptake) were defined to demonstrate how the model can be used. Islet doses were calculated for administration of $150 \mathrm{MBq}$ ${ }^{111}$ In-exendin and for $75 \mathrm{MBq}{ }^{68} \mathrm{Ga}$-exendin.

Results: The islet dose was small in all cases, ranging from 1.61 to $2.35 \mathrm{mGy}$ for ${ }^{68} \mathrm{Ga}$ and from 49.3 to $74.3 \mathrm{mGy}$ for ${ }^{111} \mathrm{In}$. The kidneys largely contributed to the isle dose (range: 49 - 81\%) and the self-dose of the islets substantially contributes to the total islet dose (up to $45 \%$ for ${ }^{111}$ In and $22 \%$ for ${ }^{68} \mathrm{Ga}$ ).

Conclusion: The calculated islet doses suggest that even repeated exendin imaging will not lead to islet toxicity. The model can already be used for calculation of islet doses in many different situations, and the design and documentation of the model enables other users not only to modify the input, but also to adjust the model to their own needs.

\section{Introduction}

The insulin producing beta cells in the islets of Langerhans in the pancreas play a key role in maintaining blood glucose levels in a normal range. In diabetes, the glucose homeostasis is disturbed, by different underlying pathologies, resulting in insufficient beta cell function. Until recently, the relation between beta cell mass (BCM) and beta cell function could not be investigated thoroughly due to the lack of a method to measure the BCM non-invasively. Therefore, various radiolabeled tracers have been developed for beta cell quantification by PET and SPECT in preclinical studies (1) and the first tracers are being translated to the clinic (2-4). One promising tracer for beta cell quantification is radiolabeled exendin, which binds specifically to the glucagon-like peptide-1 (GLP-1) receptor on the beta cells and is internalized upon receptor binding (2). This internalization and subsequen "metabolic trapping" of the tracer, labeled with a radiometal (2), leads to high target-to-background ratios

Since the islets are of vital importance for maintaining glucose homeostasis, the accumulation of radioactivity leads to concerns about radiation induced damage to the islets, especially after repeated radiotracer imaging. To ensure imaging without severely damaging the islets, a method to calculate the islet radiation dose is required.

Currently, there is no model available to calculate the radiation dose on the islets. Some attempts were made relying on organ-based macro-dosimetry to calculate the average radiation dose on the pancreas $(5,6)$. Mikkola et al (5) used the rat biodistribution of NODAGA-Exendin-4, labeled with ${ }^{64} \mathrm{Cu}$ and labeled with ${ }^{68} \mathrm{Ga}$ to estimate human whole body radiation doses and organ radiation dose with OLINDA/EXM. In a similar way, Wild et al (6) used the biodistribution of Rip1Tag2 mice for three different exendin radiopeptides. In both studies only the pancreas radiation dose was calculated assuming homogenous radioactivity distribution in the pancreas. However, the uptake in the islets is much highe compared to exocrine tissue (2), which will lead to a relatively high radiation dose on the islets compared to the exocrine tissue. Therefore the average radiation dose on the pancreas underestimates the islet radiation dose and for this reason the islets should be included as a separate part in the islet radiation dose calculation. Therefore, a dosimetry model that enables accurate calculation of the dose to the islets and information about the activity distribution in humans, as reliable input for this model, are needed.

In this work, a model based on different dosimetry methods was developed for calculation of the islet radiation dose after administration of radiolabeled exendin. In a previous clinical study, high accumulation of radiolabeled exendin in the pancreas and the kidneys was observed, while the radioactivity concentration in 
the remainder of the body was low (2). Therefore, the kidneys and pancreas are source organs in the macro-dosimetry part of the model and the remainder of the body is not included. To complete the model, also micro-dosimetry of the islets is included.

In this model the islet self-dose was calculated using Monte Carlo based micro-dosimetry, whereas the radiation doses due to kidneys and pancreas were calculated using the OLINDA/EXM (7) macro-dosimetry software. Both preclinical and clinical data on the tracer distribution were used as input for the model.

Finally, the model was applied to estimate absolute islet radiation doses and the effect of different radionuclides $\left({ }^{111} \mathrm{In}\right.$ or $\left.{ }^{68} \mathrm{Ga}\right)$ and different biological influences (male or female, low or high kidney activity, small or large islets, healthy or diabetic subjects) on the radiation doses.

\section{Materials and methods}

\section{The model}

The radiation dose to the islets is the sum of radiation doses originating from the different sources. Figure 1 shows the theoretical contributors (sources) to the islet radiation dose.

For calculation of the islet radiation dose $(D)$, S-values of each source are multiplied by the administered activity $\left(A_{0}\right)$, and the time integrated activity coefficient of the source, also called residence time $(\tau)$.

$$
D=A_{0}\left(S_{\text {pancreas } \leftarrow \text { kidneys }} \tau_{\text {kidneys }}+S_{\text {pancreas } \leftarrow \text { pancreas }} \tau_{\text {pancreas }}+S_{\text {islet } \leftarrow \text { islet }} \tau_{\text {islet }}\right)
$$

S-values, as used in OLINDA/EXM (7) and based on the methodology of the Medical Internal Radiation dose (MIRD) committee, describe the contribution of activity of one organ (source) to the radiation dose in another organ (target). When source and target are the same, the resulting radiation dose is called self-dose. For the use of S-values, it is assumed that activity is homogeneously distributed in the source and radiation dose deposition is uniform in each target organ (8). For the kidney to pancreas and pancreas self-dose we used S-values from OLINDA/EXM which were based on Monte Carlo simulations in the standard phantoms of an average male and female (9). S-values for the islets cannot be taken from OLINDA/EXM, since the islets are not defined as a separate organ. Alternatively, OLINDA/EXM provides the possibility to calculate self-doses in spheres, which is commonly used for tumors, under the assumption of sphericity. However, the smallest sphere in OLINDA/EXM (diameter: $0.13 \mathrm{~cm})(10)$ is much larger than the typical islet sizes $(50-400 \mu \mathrm{m})$ therefore new Monte Carlo simulations had to be performed for this specific application.

The source-specific factor in radiation dose calculations is the residence time. Residence times for kidneys were calculated from clinical planar scintigraphy whereas residence times for pancreas and islets were based on preclinical biodistribution, autoradiography and histological data.

The last factor necessary to calculate the radiation dose is the administered activity. Model calculations were performed using an administered activity of 150 $\mathrm{MBq}$ for ${ }^{111} \mathrm{In}$ (as used in our previous clinical study (2)), and $75 \mathrm{MBq}$ for ${ }^{68} \mathrm{Ga}$, because of the higher sensitivity of PET imaging with ${ }^{68} \mathrm{Ga}$ compared to SPECT imaging with ${ }^{11} \mathrm{In}$.

Each following section describes one part of the islet dosimetry model kidneys, pancreas and islet). For each source we describe the assumptions in which way S-values and residence times were obtained.

\section{Kidneys}

The average radiation dose in the pancreas due to the kidneys is calculated with S-values. Since the islet for which the radiation dose is calculated is part of the pancreas, the average radiation dose in the pancreas due to the kidneys, is representative for the average radiation dose in the islet due to the kidneys.

The S-values for the kidney to pancreas radiation dose calculation $\left(\mathrm{S}_{\text {pancreas } \leftarrow \text { kidney }}\right)$ were taken from OLINDA/EXM for an adult male and an adult female, for ${ }^{111}$ In and ${ }^{68} \mathrm{Ga}$ (see table 1).

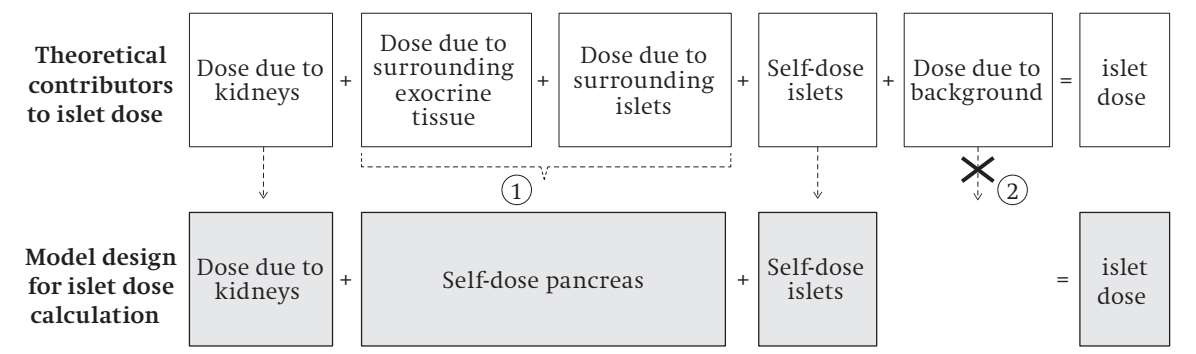

Figure 1 Structure of the islet dosimetry model. The assumptions made are indicated with numbers: 1) All islets are homogeneously distributed through the pancreas. 2) The activity in the background is very low (known clinical data) and can therefore be neglected. 
Table 1 S-values retreived from OLINDA/EXM and used in the islet dosimetry model

\begin{tabular}{|c|c|c|c|c|}
\hline & \multicolumn{2}{|l|}{${ }^{111}$ In } & \multicolumn{2}{|l|}{${ }^{68} \mathrm{Ga}$} \\
\hline & M & $\mathrm{F}$ & M & F \\
\hline $\mathrm{S}_{\text {pancreas }<\text { pancreas }}(\mathrm{mGy} / \mathrm{MBq} . \mathrm{s})$ & $1.01 \mathrm{E}-4$ & $1.12 \mathrm{E}-4$ & $1.31 \mathrm{E}-3$ & $1.45 \mathrm{E}-3$ \\
\hline $\mathrm{S}_{\text {pancreas } \leftarrow \text { kidneys }}(\mathrm{mGy} / \mathrm{MBq} \cdot \mathrm{s})$ & $1.47 \mathrm{E}-6$ & $1.77 \mathrm{E}-6$ & $3.47 \mathrm{E}-6$ & $3.97 \mathrm{E}-6$ \\
\hline
\end{tabular}

Kidney residence times were based on planar scintigraphy images from a previous clinical study (2).The protocol was approved by the Regional Ethics Review Board (CMO) and written confirmed consent was obtained from all participants. In this dosimetry study, images of five healthy volunteers acquired 0, 4, 24, 48, 96 and 168 $\mathrm{h}$ after injection of $150 \mathrm{MBq}{ }^{111}$ In-exendin- 4 were used for calculation of the kidney residence times. The amount of activity in the kidneys at the different time point and the residence time for ${ }^{111}$ In was calculated with SPRIND-software (11). For calculation of ${ }^{68} \mathrm{Ga}$ residence times, the amounts of activity were corrected for the shorter half-live, assuming similar biological behavior. Within the islet dosimetry model the average kidney residence time of these five volunteers was used as input. For simulation of humans with high or low kidney activity, the minimum or maximum calculated residence times were used as input.

\section{Self-dose pancreas}

Within the pancreas, activity in the exocrine part and islets contribute to the islet radiation dose. When assuming that the islets are homogeneously distributed throughout the pancreas and are infinitely small, the islets and the exocrine tissue contribute to the islet radiation dose as one source. The average radiation dose to the target islet due to the surrounding islets and exocrine tissue is defined as the self-dose of the pancreas. The conventional MIRD methodology assumes that the distribution of radiopharmaceuticals is uniform within the pancreas. Additionally the islet dose is enhanced by specific uptake of the labeled exendin in the islets of Langerhans.

The S-values for the pancreas self-dose $\left(S_{\text {pancreas } \leftarrow \text { pancreas }}\right)$ were taken from OLINDA/EXM for an adult male and adult female, for ${ }^{111} \mathrm{In}$ and ${ }^{68} \mathrm{Ga}$ (see table 1).

Currently, no reliable data on the accumulation of ${ }^{111}$ In-exendin in islets and exocrine tissue are available. Therefore an alternative approach using preclinica data was set up. Shortly, rat biodistribution data was used to calculate absolute pancreatic uptake, histological data were used to calculate the fraction of beta cells in the rat pancreas, and the autoradiography provided the uptake ratio of exocrine and islet tissue. This information was used for calculation of the residence time of islet and exocrine tissue in human.

Here, the animal experiment, use of the animal data for calculation of the pancreas residence times and the conversion to the human situation are described more extensively.

Nine female Biobreeding Diabetes Prone (BBDP) rats (5-7 weeks old) (Biomedica Research Models, Worchester, MA, USA), were euthanized two hours after injection of $18.5 \pm 1.78 \mathrm{MBq}{ }^{111}$ In-exendin-3 (peptide dose 20pmol). Pancreata were dissected and activity in the pancreas for each rat was measured in a gamma counter (Wallac 1480 wizard, Perkin Elmer, Waltham, MA, USA). The pancreata were fixed in formalin, embedded in paraffin and sliced in $4 \mu \mathrm{m}$ sections for autoradiography and histological evaluation. The animal experiments were approved by the animal welfare committee of the Radboud University Nijmegen.

For autoradiography, tissue sections were exposed to a phosphor imaging plate and developed after 7 days using a radioluminography laser imager (Fuji Film BAS 1800 II system, Raytest, Straubenhardt, Germany). Autoradiography images were used to calculate the ratio between uptake in the exocrine tissue and the islets. The autoradiography images were exported from the AIDA software (Raytest) for further processing in MATLAB (version R2011a, The MathWorks, Inc., USA).

The same sections were stained for the presence of insulin and scanned with an automated microscope as described previously (2). Figure 2 shows the strong correlation of the autoradiography with the position of the islets in the histological image of the same section. Thresholding was performed on pancreatic tissue in order to semi-automatically determine the total pancreatic area using Image (version 1.47, National Institutes of Health, USA). Subsequently, insulin positive islets were delineated in iVision (iVision-Mac ${ }^{\mathrm{TM}} 4.0 .16$, Biovision Technologies, USA) and mask images of both delineated islets and total pancreatic area were saved The fraction of islets ) in the pancreas was calculated by dividing the insulin positive pixel (islet mask) area by the total pancreatic pixel area (tissue mask) per rat.

In MATLAB, the autoradiographic images ( $\mathrm{n}=3$ per rat) were inverted and compared with the mask images of the islets and the pancreas tissue of the same slice. Therefore the autoradiography images were scaled, rotated and translated to ensure optimal registration.

For calculation of the activity in the background (outside the tissue) and in the exocrine tissue, three regions were manually selected (see figure $3 \mathrm{~A}$ and $\mathrm{B}$ ) to avoid inclusion of islets which were not delineated, or inclusion of contamination in the background.

The registration of the masks and autoradiography images and selection of exocrine and background regions was performed by two researchers (IK and WW) and the results were averaged. The registration quality of histological and autora- 


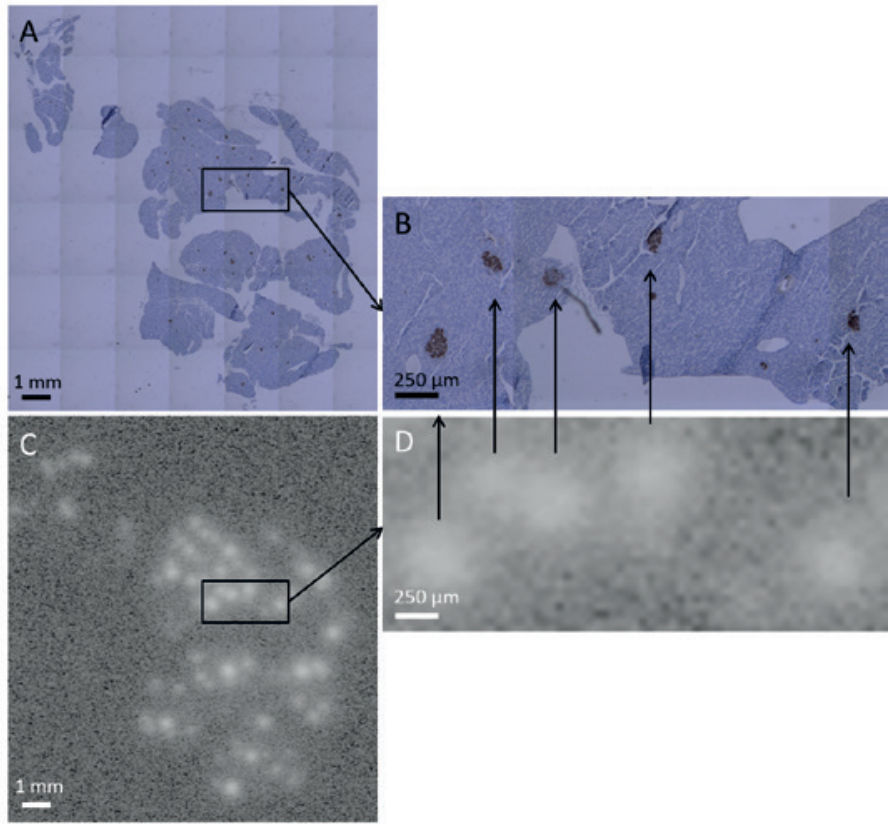

Figure 2 The position of the islets, immunohistochemically stained for insulin in brown graphy (C and D). B and D are magnifications of the areas indicated with a box in A and C.

diographic images was scored from 1-5 (1 being very poor registration and 5 being excellent registration), and only images with a score $3-5$ ( $80 \%$ of the images) were included.

The pixel value in the exocrine tissue was corrected for the background:

$$
A_{e x / p i x}=A_{e x, u / p i x}-A_{b g / p i x}
$$

Where $A_{b g / p i x}$ is the average background pixel value, $A_{e x, \mu p \text { ix }}$ the uncorrected average pixel value in the exocrine tissue and $A_{\text {expix }}$ the corrected average pixel value in the exocrine tissue.

Figure $3 \mathrm{C}$ and D show that in the autoradiography, activity originating from the islets is also projected in the surrounding of the islets. This spill over is caused by the distance between the tissue slice and the phosphor imaging plate. Delineated islets were dilated (see figure 3E), to include spill over originating from the islets in calculation of the ratio between islets and exocrine tissue. The activity in the (A and B), correlates with the accumulation of ${ }^{111}$ In-exendin in islets visualized by autoradio-

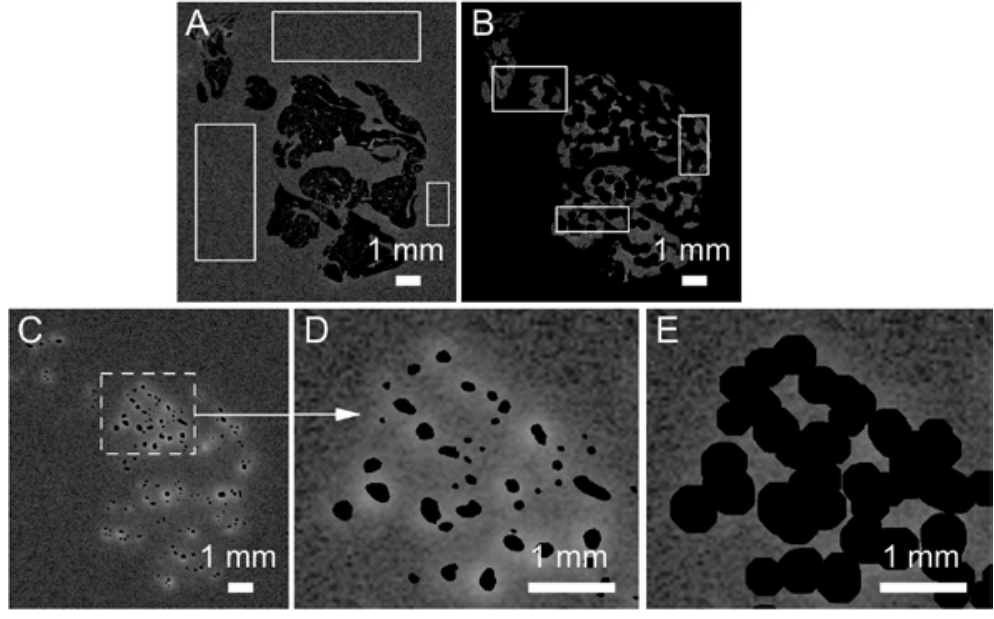

Figure 3 (A) Selection of areas (rectangles) for calculation of the background activity in an autoradiography image with a mask for pancreatic tissue. (B) shows the selection of regions (rectangles) for calculation of the uptake in the exocrine tissue, in the autoradiography image, with a mask which only shows exocrine tissue (dilated islets and background are excluded). (C) shows the autoradiography image with the islets in black, and demonstrates the projection of islet activity outside the islets. (D) shows a magnification of the islets with the autoradiography. (E) visualizes how dilation of the delineated islets enables inclusion of all activity originating from the islets, as no spill over is visible.

dilated part (rim) was corrected for exocrine tissue activity, and included in the calculated islet pixel value $\left(A_{\text {islppix }}\right)$ :

$$
A_{i s l / p i x}=A_{i s l u / p i x}+\frac{\operatorname{Area}_{\text {rim }}\left(A_{\text {rim }, u / p i x}-A_{\text {ex }, u / p i x}\right)}{\text { Area }_{i s l}}-A_{b g / p i x}
$$

Where $A_{i s, u p p i x}$ is the uncorrected average pixel value in the islet, Area $a_{\text {rim }}$ is the number of pixels in the dilation area surrounding the islets, $A$ is the uncorrected average pixel value in the dilation area, Area $_{i \mathrm{is}}$ is the number of pixels in the non-dilated islet area.

Finally, this results in the uptake ratio $(R)$ between islet and exocrine tissue, defined as:

$$
R=\frac{A_{i s l / p i x}}{A_{\text {ex/pix }}}
$$


For calculation of the residence times we assumed that immediately after injection the activity is instantaneously distributed to its final position in the body, and from this moment all activity in the islets remains in the islets, and all activity in the exocrine tissue remains in the exocrine tissue.

To calculate the residence times for the human pancreas, first residence times for the rat pancreas were calculated. The fraction injected dose in the rat pancreas $\left(f_{I D \text { pand }}\right)$, determined by dividing the pancreas activity (from the biodistribution data) by the administered activity, was used to calculate the residence time for the whole pancreas $\left(\tau_{\text {panc rat }}\right)$

$$
\tau_{\text {panc rat }}=f_{I D, \text { panc }} \cdot \int_{0}^{\infty} e^{-\lambda t} d t=f_{I D, p a n c} \cdot \frac{1}{\lambda}
$$

Where $\lambda$ is the physical decay constant.

The pancreas residence time per voxel ( $\left.\tau_{\text {panc rattvox }}\right)$ was calculated from the residence time for the whole pancreas, including the islets, divided by the number of voxels in the pancreas $\left(\#_{v o x}\right)$. The number of voxels was calculated from the pancreas weight, tissue density (assumed $\left.1.079 \mathrm{~g} / \mathrm{cm}^{3}(12)\right)$ and voxel volume $\left(50 \times 50 \times 4 \mu \mathrm{m}^{3}\right)$.

$$
\tau_{\text {panc rat } / \text { vox }}=\frac{\tau_{\text {panc rat }}}{\#_{\text {vox }}}
$$

The islet fraction $\left(f_{i s}\right)$ in the pancreas and the activity ratio between islet and exocrine tissue $(R)$, were used to separate this whole pancreas voxel residence time into a voxel residence time for exocrine tissue $\left(\tau_{\text {exo rat vox }}\right)$ and for islet tissue $\left(\tau_{\text {is rat rox }}\right)$ :

$$
\tau_{\text {exo rat } / \text { vox }}=\frac{\tau_{\text {panc rat } / \text { vox }}}{f_{\text {isl }} \cdot R+\left(1-f_{\text {isl }}\right)}
$$

$$
\tau_{\text {isl rat } / \text { vox }}=\frac{\tau_{\text {panc rat } / \text { vox }}-\tau_{\text {exo rat } / v o x}\left(1-f_{\text {isl }}\right)}{f_{\text {isl }}}
$$

Residence times per voxel for both exocrine tissue and islets were translated to humans with the following formula:

$$
\tau_{\text {human } / \text { vox }}=\tau_{\text {rat } / \text { vox }}\left(\frac{W_{T B, \text { rat }}}{W_{T B, \text { human }}}\right)
$$

With $W_{\text {TBrote }}$, the total body weight of the rat, and $W_{\text {TBhuman }}$, the total body weight of the human (male $70.0 \mathrm{~kg}$, female $56.9 \mathrm{~kg}$ ).

Subsequently the residence time for the whole human pancreas was calculated. The pancreas includes exocrine tissue and islets, therefore besides pancreas volume, also the percentage of islets influences the pancreas residence time. The sum of human residence times of the exocrine part $\left(\tau_{\text {exo human }}\right)$ and the islets in the pancreas $\left.\tau_{\text {is human }}\right)$ give the residence time of the human pancreas $\left(\tau_{\text {panc human }}\right)$ required for calculation of the self-dose:

$$
\tau_{\text {panc human }}=\tau_{\text {exo human }}+\tau_{\text {isl human }}
$$

Where:

$$
\tau_{\text {exo human }}=\tau_{\text {exo human } / \text { vox }} \frac{\left(1-f_{\text {isl }}\right) V_{\text {panc }}}{V_{\text {vox }}}
$$

$$
\tau_{\text {isl human }}=\tau_{\text {isl human } / \text { vox }} \frac{f_{\text {isl }} V_{\text {panc }}}{V_{\text {vox }}}
$$

Where $f_{\text {iss }}$ is the fraction of islets in the pancreas, $V_{\text {panc }}$ the volume of the pancreas, and $V_{\text {vox }}$ the voxel volume $\left(50 \times 50 \times 4 \mu \mathrm{m}^{3}\right) . \tau_{\text {exo human/vox }}$ and $\tau_{\mathrm{s}}$ were calculated with formula 9.

Within the islet dosimetry model normally the residence times are calculated assuming 3\% islets in the pancreas. This value can be varied to simulate lower or higher percentages of islets, mimicking natural variation in beta cell percentage in healthy individuals as well as beta cell loss in diabetic patients. Furthermore the weight of the pancreas, used for calculation of the residence time, is assumed to be similar to the standard male $(94.3 \mathrm{~g}$ ) or female ( $85.0 \mathrm{~g})$. 


\section{Self-dose islets}

For calculation of S-values for ${ }^{111} \mathrm{In}$ and ${ }^{68} \mathrm{Ga}$ in spheres with sizes that cover the range of the islet sizes, new Monte Carlo simulations were performed. The method for these Monte Carlo simulations were described previously (10), although for the present calculations only MCNP5 code was used and the comparison with results calculated with the EGS4 code was skipped. For the Auger electrons, that have an energy $<1 \mathrm{keV}$, complete local absorption was assumed. For the electrons with an energy $>1 \mathrm{keV}$ and for photons, 10 million Monte Carlo simulations were performed for spheres with diameters of 50,100, 200 and $400 \mu \mathrm{m}$. The particle spectra from the MIRD-07 database were used as input (13).

These simulations lead to $\mathrm{S}$-values for the islet self-dose $\left(\mathrm{S}_{\text {ist }}\right)$ for different islet sizes, for ${ }^{111} \mathrm{In}$ and ${ }^{68} \mathrm{Ga}$ (see table 2).

Within this islet dosimetry model, these S-values are multiplied by the timeintegrated activities in each source region. The calculation of the residence times is performed as described for the pancreas above. The time-integrated activity of the islet is obtained by product of number of voxels within the islets, the voxel residence time for the islets and the injected activity.

The islet dosimetry model includes islets with diameters of 50, 100, 200 and $400 \mu \mathrm{m}$.

Table 2 Results of small sphere Monte Carlo simulations that were included in the model.

\begin{tabular}{|l|l|l|}
\hline $\begin{array}{l}\text { islet diameter } \\
(\mu \mathrm{m})\end{array}$ & $\begin{array}{l}{ }^{11} \mathrm{In} \\
\text { S-value (mGy/MBq.s) }\end{array}$ & $\begin{array}{l}{ }^{68} \mathrm{Ga} \\
\text { S-value (mGy/MBq.s) }\end{array}$ \\
\hline 50 & 14744.3 & 8500.5 \\
\hline 100 & 2231.4 & 2050.8 \\
\hline 200 & 387 & 512.6 \\
\hline 400 & 77.6 & 131.2 \\
\hline
\end{tabular}

\section{Use of the model}

The model we developed for calculation of the radiation dose to the islets was incorporated in an Excel file, which facilitates data input and can generate results for various situations. This file is available as supplementary material.

To demonstrate the application of the model, several examples are described. The examples and the model are used to visualize changes in the islet radiation doses due to: (I) the effect of using ${ }^{68} \mathrm{Ga}$ instead of ${ }^{111} \mathrm{In}$, (II) the difference between male and female, (III) the effect of different amounts of activity in the kidney, (IV) the difference between small and large islet sizes and (V) differences between diabetic and healthy subjects (e.g. low versus high percentage of beta cells).

Details about the model input for the different examples are given in table 3.

Shortly, the input for example (I), in which the islet radiation dose is calculated for a male with an islet diameter of $100 \mu \mathrm{m}$ and $3 \%$ of the pancreas consists of islets. Example I is also used as reference for the other examples.

In example II, in which the islet radiation dose for ${ }^{111} \mathrm{In}$ and ${ }^{68} \mathrm{Ga}$ is calculated for a emale, the gender is changed which changes the S-values for the kidney to pancreas radiation dose and for the pancreas self-dose, and the pancreas weight is $85 \mathrm{~g}$ (compared to $94.3 \mathrm{~g}$ for male).

The residence time of the kidneys are altered in example III to simulate a high kidney uptake ( $=63.71 \mathrm{~h}$ ) and a low kidney uptake $(=35.6 \mathrm{~h})$. Islet radiation doses are calculated for the use of ${ }^{111}$ In in male.

In example IV the islet radiation doses for small islets (50 $\mu \mathrm{m}$ diameter) and large islets (400 $\mu \mathrm{m}$ diameter) are calculated for ${ }^{111} \mathrm{In}$ and ${ }^{68} \mathrm{Ga}$ in male

The last example, example $\mathrm{V}$, simulates a diabetic patient. The percentage of islets in the pancreas is reduced to $0.3 \%$, as compared to $3 \%$ in the reference situation, and islet radiation doses for male are calculated for ${ }^{111} \mathrm{In}$ and ${ }^{68} \mathrm{Ga}$.

\section{Results}

The model developed for islet dosimetry was used for calculation of the dose in different situations. Results of all examples are given in table 3 . Here, we describe the main results.

In example I ( ${ }^{111} \mathrm{In}$ versus ${ }^{68} \mathrm{Ga}$ ), the total islet dose is much lower for ${ }^{68} \mathrm{Ga}$ than for ${ }^{111} \mathrm{In}(1.97 \mathrm{mGy}$ versus $60.1 \mathrm{mGy}$ ). The relative contribution of the islet self-dose to the total islet dose is smaller for ${ }^{68} \mathrm{Ga}$ than for ${ }^{111} \mathrm{In}$ (6.3\% versus $27 \%$ ), whereas the relative contribution of the kidneys to the islet dose in the same range for ${ }^{111} \mathrm{In}$ and ${ }^{68} \mathrm{Ga}(65 \%$ and $67 \%$ ) (see figure $4 \mathrm{~A}$ and $\mathrm{B}$ ).

Example II shows that females have higher total islet doses ( $24 \%$ for ${ }^{111} \mathrm{In}, 19 \%$ for ${ }^{68} \mathrm{Ga}$ ) compared to male. Although the absolute dose is higher for female, the relative contribution of all sources is similar.

In example III, a low $\left(\tau_{\text {kidneys }}=35.6 \mathrm{~h}\right)$ and a high kidney uptake $\left(\tau_{\text {kidneys }}=63.7 \mathrm{~h}\right)$ was simulated. The islet dose due to the kidneys was $28.3 \mathrm{mGy}$ and $50.6 \mathrm{mGy}$ respectively. The contribution of the remainder of the pancreas and the self-dose do not change, therefore the difference between the low and high-uptake cases in overall islet dose is $45 \%$ ( $49.3 \mathrm{mGy}$ versus $71.6 \mathrm{mGy}$ ).

By changing islet size, as in example IV, only the self-dose of the islets is influenced. For ${ }^{111} \mathrm{In}$ the self-dose was $13.3 \mathrm{mGy}$ for islets with a diameter of $50 \mu \mathrm{m}$ 
and $35.9 \mathrm{mGy}$ for $400 \mu \mathrm{m}$. The ${ }^{68} \mathrm{Ga}$ self-dose was $0.0644 \mathrm{mGy}$ for $50 \mu \mathrm{m}$ islets and $0.509 \mathrm{mGy}$ for $400 \mu \mathrm{m}$. For the large islets this results in a high relative contribution of the islet self-dose to the total islet dose ( $45 \%$ for ${ }^{111} \mathrm{In}$ and $21 \%$ for ${ }^{68} \mathrm{Ga}$ ). Compared to $50 \mu \mathrm{m}$ islets, the total islet dose in $400 \mu \mathrm{m}$ islets is increased with $39 \%$ for ${ }^{111} \mathrm{In}$ and $23 \%$ for ${ }^{68} \mathrm{Ga}$.

To simulate diabetic patients (example V) the percentage islets in the pancreas was reduced from $3 \%$ to $0.3 \%$. This strongly influences the self-dose of the pancreas due to surrounding islets: for ${ }^{111} \mathrm{In}$ in male the self-dose drops from $3.65 \mathrm{mGy}$ to $0.365 \mathrm{mGy}$ and for ${ }^{68} \mathrm{Ga}$ from $0.397 \mathrm{mGy}$ to $0.0397 \mathrm{mGy}$ in a diabetic patient (also
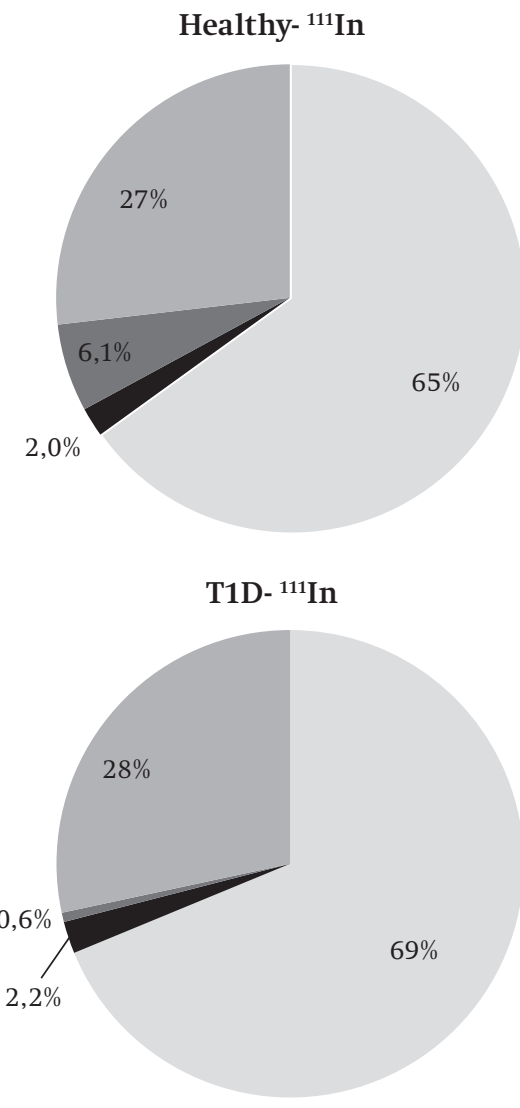

Figure 4 Relative contributions of the kidneys ( $($ ), remainder of the pancreas (exocrine tissue (I) and surrounding islets (I)) and islet self-dose (I) to the total islet radiation dose for ${ }^{111} \mathrm{In}$ and ${ }^{68} \mathrm{Ga}$. A and $\mathbf{B}$ show the dose contributions for ${ }^{111} \mathrm{In}$ and ${ }^{68} \mathrm{Ga}$ for a healthy male $\mathrm{C}$ and $\mathrm{D}$ show the ${ }^{111} \mathrm{In}$ and ${ }^{68} \mathrm{Ga}$ dose contributions for male with type 1 diabetes (T1D). see figure 4). Because a larger percentage of the pancreas consists of exocrine tissue in diabetic patients, the self-dose due to the exocrine tissue minimally increases $(<3 \%)$.

\section{Discussion}

In this study we developed a model for calculation of the radiation dose to the islets of Langerhans after administration of radiolabeled exendin. This innovative model is based on macro-dosimetry (for pancreas and kidney contribution) and micro-dosimetry methods (islet self-dose), and relies for the calculations, not only on preclinical, but also on clinical data. To simulate different biological circumstances (for tailoring the dosimetry for a specific patient group), the mode can mimic various situations, such as diabetes, different radionuclides and differences in gender.

In all situations the islet dose was very low (maximum $74.3 \mathrm{mGy}$ ) compared to the dose inducing islet damage (10 Gy) with external beam radiation therapy (14) especially when considering the lower biological effectivity of radiation dose due to internal radiation. This indicates that multiple investigations with radiolabeled exendin can be performed without any risk to induce islet damage. For confirmation, islet damage could be measured after exposing isolated islets of Langerhans in vitro to the found radiation doses. In order to minimize islet doses, the use of ${ }^{68} \mathrm{Ga}$-exendin is preferred over ${ }^{111} \mathrm{In}$-exendin.

The contribution of the activity in the kidneys to the total islet dose is considerable, ranging from $49 \%$ for $400 \mu \mathrm{m}$ islets in male administered with ${ }^{111} \mathrm{In}$ exendin, to even $81 \%$ in diabetic male administered with ${ }^{68} \mathrm{Ga}$-exendin. Therefore reducing kidney activity (e.g. with gelofusine (6)) would not only increase image quality, but would also be beneficial for the islet dose.

Wild et al. (6) calculated the pancreas doses for ${ }^{111} \mathrm{In}$-exendin and ${ }^{68} \mathrm{Ga}$-exendin. For ${ }^{111} \mathrm{In}$-exendin a pancreas dose of $0.7 \mathrm{mGy} / \mathrm{MBq}(105 \mathrm{mGy}$ for $150 \mathrm{MBq})$ and for ${ }^{68} \mathrm{Ga}$-exendin $0.2 \mathrm{mGy} / \mathrm{MBq}(15 \mathrm{mGy}$ for $75 \mathrm{MBq}$ ) was found. Considering that for these calculations mice data were used and islet self-dose was not included, it can be concluded that although their results are in a somewhat higher range, they are in line with the doses found in our study. In our islet dosimetry model, the self-dose of the islets was added as a separate contributor. In all our examples, contribution of the activity in the islet itself to the total islet dose was considerable (with the largest contribution in large islets, for ${ }^{111} \mathrm{In} 45 \%$, and for $\left.{ }^{68} \mathrm{Ga} 22 \%\right)$. This indicates that the accumulation of activity in the islets substantially contributes to the pancreas dose, and therefore has to be included in the dose calculation.

The model we have developed is a useful tool for calculation of islet doses. The current model is well documented, and can therefore be further optimized by 
Table 3 Details and results of the examples. The results of example I are also used as reference for example II and $\mathrm{V}$.

\begin{tabular}{|c|c|c|c|c|c|c|c|c|c|c|c|c|}
\hline & Example I & & Example II & & Example III & & Example IV & & & & Example V & \\
\hline Input & ${ }^{111}$ In & ${ }^{68} \mathrm{Ga}$ & $\begin{array}{r}\text { Female } \\
{ }^{111} I n\end{array}$ & $\begin{array}{l}\text { Female } \\
{ }^{68} \mathrm{Ga}\end{array}$ & $\begin{array}{r}\text { High } \\
\text { kidney } \\
\text { activity }\end{array}$ & $\begin{array}{r}\text { Low } \\
\text { kidney } \\
\text { activity }\end{array}$ & $\begin{array}{r}\text { Small } \\
\text { islets }\end{array}$ & Large islets & Small & Large & $\begin{array}{r}\text { Type } 1 \\
\text { diabetes } \\
{ }^{111} I n\end{array}$ & $\begin{array}{r}\text { Type } 1 \\
\text { diabetes } \\
{ }^{68} \mathrm{Ga}\end{array}$ \\
\hline gender & M & M & $\mathrm{F}$ & $\mathrm{F}$ & M & $\mathrm{M}$ & M & M & M & M & M & M \\
\hline Weight pancreas (g) & 94.3 & 94.3 & 85 & 85 & 94.3 & 94.3 & 94.3 & 94.3 & 94.3 & 94.3 & 94.3 & 94.3 \\
\hline Isotope & ${ }^{111} \mathrm{In}$ & ${ }^{68} \mathrm{Ga}$ & ${ }^{111} \mathrm{In}$ & ${ }^{68} \mathrm{Ga}$ & ${ }^{111} \mathrm{In}$ & ${ }^{111} \mathrm{In}$ & ${ }^{111} \mathrm{In}$ & ${ }^{111} \mathrm{In}$ & ${ }^{68} \mathrm{Ga}$ & ${ }^{68} \mathrm{Ga}$ & ${ }^{111} \mathrm{In}$ & ${ }^{68} \mathrm{Ga}$ \\
\hline Injected activity (MBq) & 150 & 75 & 150 & 75 & 150 & 150 & 150 & 150 & 75 & 75 & 150 & 75 \\
\hline Islet size (um) & 100 & 100 & 100 & 100 & 100 & 100 & 50 & 400 & 50 & 400 & 100 & 100 \\
\hline Residence time islet (hour) & $1.34 \mathrm{E}-8$ & $2.24 \mathrm{E}-10$ & $1.73 \mathrm{E}-8$ & $2.90 \mathrm{E}-10$ & $1.34 \mathrm{E}-8$ & $1.34 \mathrm{E}-8$ & $1.67 \mathrm{E}-9$ & 8.56E-7 & $2.80 \mathrm{E}-11$ & $1.44 \mathrm{E}-8$ & $1.34 \mathrm{E}-8$ & $2.24 \mathrm{E}-10$ \\
\hline Percentage islets in pancreas $(\%)$ & 3 & 3 & 3 & 3 & 3 & 3 & 3 & 3 & 3 & 3 & 0.3 & 0.3 \\
\hline Residence time pancreas (hour) & 0.0895 & 0.0015 & 0.1045 & 0.0018 & 0.0895 & 0.0895 & 0.0895 & 0.0895 & 0.0015 & 0.0015 & 0.0298 & 0.0005 \\
\hline Residence time kidneys (hour) & 49.6 & 1.40 & 49.3 & 1.40 & 63.7 & 35.6 & 49.3 & 49.3 & 1.40 & 1.40 & 49.3 & 1.4 \\
\hline & & & & & & & & & & & & \\
\hline \multicolumn{13}{|l|}{ Results } \\
\hline Pancreas dose due to kidneys (mGy) & 39.1 & 1.31 & 47.1 & 1.50 & 50.6 & 28.3 & 39.1 & 39.1 & 1.31 & 1.31 & 39.1 & 1.31 \\
\hline $\begin{array}{r}\text { Self-dose pancreas (mGy) -exocrine } \\
\text { part }\end{array}$ & 1.23 & 0.133 & 1.59 & 0.172 & 1.23 & 1.23 & 1.23 & 1.23 & 0.133 & 0.133 & 1.26 & 0.137 \\
\hline Self dose pancreas (mGy) - islets & 3.66 & 0.397 & 4.73 & 0.513 & 3.65 & 3.65 & 3.65 & 3.65 & 0.397 & 0.397 & 0.365 & 0.0397 \\
\hline Self dose pancreas (total) & 4.88 & 0.531 & 6.32 & 0.686 & 4.88 & 4.88 & 4.88 & 4.88 & 0.531 & 0.531 & 1.63 & 0.177 \\
\hline Self-dose islet (mGy) & 16.1 & 0.124 & 20.9 & 0.161 & 16.1 & 16.1 & 13.3 & 35.9 & 0.0644 & 0.509 & 16.1 & 0.124 \\
\hline Total islet dose (mGy) & 60.1 & 1.97 & 74.3 & 2.35 & 71.6 & 49.3 & 57.3 & 79.9 & 1.91 & 2.35 & 58.8 & 1.61 \\
\hline
\end{tabular}

other users. For example, it would be of great interest to include more clinical dat and calculate gender-specific residence times for kidneys and pancreas. This requires investigation of a larger cohort and absolute quantification of the pancreas uptake in human (including ex vivo measurements).

Another interesting adjustment of the model would be to separate the pancreas in different compartments. In the current model an average islet dose is calculated, while the actual islet dose might be higher for islets in close proximity of the kidneys, compared to islets further away from the kidneys, or at the edge of the pancreas. By separating the pancreas in different compartments, islet radiation doses for separate areas could be calculated. This requires calculation of S-factors for these compartments in relation to the kidneys and to each other.
Separation of the pancreas in different compartments would also enable simulation of inhomogeneous islet densities within the pancreas, as seen in human (15)

\section{Conclusion}

The developed model can be used for calculation of islet doses in many different situations, as we showed for five examples. The results demonstrate that the use of ${ }^{68} \mathrm{Ga}$ instead of ${ }^{111}$ In seriously reduces the islet dose. The islet self-dose is substantial, and should therefore be included in islet dose calculations. 
Other users are able to not only modify the input, but also adjust the model to their own needs. This enables even broader application of this islet dosimetry model.

\section{Acknowledgements}

The authors thank Cathelijne Frielink, Lieke Joosten, Desiree Bos and Hanneke

Peeters for their work in the animal experiments, staining and scanning of the coupes and delineation of the islets.

\section{References}

ges of human beta cell mass quantificatio Diabetologia. 2014;57(5):856-9. Epub 2014/03/07.

2. Brom M, Woliner-van der Weg W, Joosten L, Frielink C, Bouckenooghe T, Rijken P, et al. Non-invasive quantification of the beta cell mass by SPECT with ${ }^{11}$ In-labelled exendin. Diabetologia. 2014;57(5):9509. Epub 2014/02/04.

3. Eriksson O, Espes D, Selvaraju RK, Jansson E, Antoni G, Sorensen J, et al. The Positron Emission Tomography ligand [11C]5-Hydroxy-Tryptophan can be used as a surrogate marker for the human endocrine pancreas. Diabetes. 2014. Epub 2014/05/23.

4. Normandin MD, Petersen KF, Ding YS, Lin SF, Naik S, Fowles K, et al. In Vivo Imaging of Endogenous Pancreatic beta-Cell Mass in Healthy and Type 1 Diabetic Subjects Using F-18-Fluoropropyl-Dihydrotetrabenazine and PET. Journal of Nuclear Medicine. 2012;53(6):908-16.

5. Mikkola K, Yim CB, Fagerholm V, Ishizu T, Elomaa VV, Rajander J, et al. 64Cu- and 68Ga-labelled [Nle(14),Lys(40)(Ahx-NODAGA)NH2]-exendin-4 for pancreatic beta cell imaging in rats. Molecular imaging and biology : MIB : the official publication of the Academy of Molecular Imaging 2014;16(2):255-63. Epub 2013/10/09.

6. Wild D, Wicki A, Mansi R, Behe M, Keil B, Bernhardt P, et al. Exendin-4-based radiopharmaceuticals for glucagonlike peptide-1 receptor PET/CT and SPECT/CT. Journal of nuclear medicine. 2010;51(7):1059 67. Epub 2010/07/03.

7. Stabin MG, Sparks RB, Crowe E. OLINDA/EXM: the second-generation personal computer software for internal dose assessment in nuclear medicine. Journal of nuclear medicine. 2005;46(6):1023-7. Epub 2005/06/07.

8. Grimes J, Celler A. Comparison of internal dose estimates obtained using organ-level, voxel $\mathrm{S}$ value, and Monte Carlo techniques. Medical physics. 2014;41(9):092501. Epub 2014/09/05.

9. Cristy M, Eckerman KF, Group MaDR. Specific absorbed fractions of energy at various ages from internal photon sources Oak Ridge, TN: Oak Ridge National Laboratory, 1987 Contract No: ORNL/ TM-8381 V1-V7.

10. Stabin MG, Konijnenberg MW. Re-evaluation of absorbed fractions for photons and electrons in spheres of various sizes. Journal of nuclear medicine. 2000;41(1):149-60. Epub 2000/01/27.

11. Visser E, Postema E, Boerman O, Visschers J, Oyen W, Corstens F. Software package for integrated dat processing for internal dose assessment in nuclear medicine (SPRIND). European journal of nuclear medicine and molecular imaging. 2007;34(3):413-21. Epub 2006/11/16.

12. Eckhard M, Brendel MD BrandhorstD Brandhorst $H$, Bretzel RG Can the density of native pancreatic tissue slices predict human islet isolation and purification outcome? Transplantation proceedings. 2004;36(9):2845-8. Epub 2004/12/29.
3. Eckerman KF, Endo A. MIRD: Radionuclide Data and Decay Schemes. 1st ed. Reston, VA: Society of Nuclear Medicine; 2008

14. de Vathaire F, El-Fayech C, Ben Ayed FF, Haddy N, Guibout C, Winter D, et al. Radiation dose to the pancreas and risk of diabetes mellitus in childhood cancer survivors: a retrospective cohort study. The Lancet Oncology. 2012:13(10):1002-10. Epub 2012/08/28.

15. Wang X Misawa R Zielinski MC, Cowen P.JoJ, Periwal V, et al Regional differences in islet distributio in the human pancras preferential beta-cell loss in the head region in patients with type 2 diabetes. PloS one. 2013;8(6):e67454. Epub 2013/07/05. 
General discussion, answers to the research questions and future perspectives 


\section{General discussion, answers to the research questions and future perspectives}

Clinical application of quantitative single photon emission computed tomography (SPECT) provides the opportunity for further patient tailoring of treatments. It enables dosimetry for gamma-emitting radionuclides, which is useful for treatment planning in radioimmunotherapy and studying dose-response relationships. Also it is a valuable diagnostic tool by facilitating three-dimensional investigation of the distribution of a gamma-emitting radionuclides in vivo. Previously technical challenges limited the use of quantitative SPECT, but especially developments in reconstruction and correction methods currently enable to cope with these challenges, and to use SPECT in a quantitative manner. This thesis focuses on how the challenges can be coped with, to enable using quantitative SPECT for dosimetry and beta cell quantification.

In chapter 1 it was shown that the challenges that should be considered for quantitative use of SPECT mainly origin from the physics in the image acquisition. Fortunately, the availability of advanced reconstruction algorithms currently allows, scatter, attenuation and collimator corrections, and therefore enable quantitative use of SPECT images. It also demonstrated that careful consideration of every step in preparation, acquisition, reconstruction and analysis of imaging data is required for reliable answering of clinical questions with quantification and dosimetric methods.

In chapter $\mathbf{2}$ accuracy of individualized dosing, based on pre-therapy administration with ${ }^{111}$ In-peptide, as surrogate for the pre-targeted radioimmunotherapy (PRIT) with ${ }^{177}$ Lu-peptide, was investigated. The relatively uncomplicated blood-based dosimetry and planar image-based dosimetry methods were applied to calculate the amount of ${ }^{177} \mathrm{Lu}$ that can be safely administered for PRIT. The study showed that with this approach indeed high amounts of activity can safely be administered with only two out of twenty patients developing grade 3-4 platelet toxicity. However, only blood-based and planar image based dosimetry was applied during this study, it clearly demonstrates that pre-therapeutic administration of surrogate radionuclide, and subsequent dosimetry is effective for individual dosing.

Currently, integrated SPECT-computed tomography (CT) and more advanced reconstruction and dosimetry methods are available, which enable potentially even more reliable predictive dosimetry based on three-dimensional images. 
One of the methods relying on three-dimensional images that can be useful for predictive bone marrow dosimetry was used in chapter 3 . In this work, 3-dimensional radiobiologic dosimetry (3D-RD) was applied for retrospective dosimetry for further investigation of the bone marrow toxicity that some patient developed in the PRIT study. We developed a method for using this Monte Carlo based dosimetry to calculate the bone marrow dose based on the activity in lumba vertebrae, and compared the results with blood-based and planar image-based bone marrow doses. 3D-RD based red bone marrow (RBM) dosimetry showed to be a more sensitive predictor for selection of patients showing any grade of RBM toxicity than planar image-based or blood-based dosimetry. This is in line with the observation that different patients in this study showed accumulation of radioactivity in the RBM: with blood-based dosimetry the accumulation is not taken into account and planar image-based RBM dosimetry is based on the activity in the skull. However, this is a faster and less complicated method than 3D-RD, the skull does not seem to be as reliable as the lumbar vertebrae for prediction of toxicity.

A next step in the validation of 3D-RD for prediction of RBM toxicity would be to use ${ }^{111}$ In-peptide based SPECT images for prediction of the ${ }^{177} \mathrm{Lu}$ radiation doses to the bone marrow. This would assess the predicting value of 3D-RD RBM dosimetry for bone marrow toxicity due to this therapy.

In addition to the RBM dosimetry, ${ }^{177} \mathrm{Lu}$ tumor-to-RBM dose ratios were calculated and compared with simulated ${ }^{90} \mathrm{Y}$, to estimate the benefit of using ${ }^{90} \mathrm{Y}$ instead of ${ }^{177} \mathrm{Lu}$. Despite the increase in tumor-to-RBM dose ratio with ${ }^{90} \mathrm{Y}$, tumor doses remained disappointingly low. Although this could be interpreted as a negative result, it was very useful in planning of future research in this field: similar clinical study with ${ }^{90} \mathrm{Y}$ was disregarded.

This example demonstrates the benefit of quantification of SPECT images for dosimetry purposes, combined with simulation of therapy with other radionuclides When a change in protocol is considered, predictive dosimetry can provide relevant results for estimation of changes in benefits and risks. Therefore SPECT-based predictive dosimetry is useful in estimating if and how the protocol should be changed (another amount of administered activity or use of a different radionuclide).

For this work we used 3D-RD, which bases its dosimetric calculations on Monte Carlo simulations. Although Monte Carlo based dosimetry is time-consuming, compared to faster commercially available dosimetry software (e.g. STRATOS of Philips), 3D-RD's ability to shape the method in the required way is valuable. In this work, it enabled using the dose rates of different volumes of interest (the lumbar vertebrae as delineated on each SPECT image) for calculation of the dose. Application of such a specific method is generally more complicated, or impossible, with more rigid commercially software.
For routine clinical dosimetry, the use of Monte Carlo simulations and thus 3D-RD is not the most user-friendly method. It puts high demands on the input of the users and therefore is too sensitive for mistakes, also a faster method for dosimetry (e.g. S-value based like OLINDA/EXM, or using dose-volume-kernels like STRATOS) is preferred. A system integrated in the software used for acquisition and reconstruction, also providing methods for calibration and quality control of the camera would reduce the risk of user-induced mistakes. Finally this will facilitate dosimetry by non-experts.

In chapter 4 and $\mathbf{5}$, SPECT is used for quantification of the pancreatic beta cells Without quantitative use of SPECT or PET, non-invasive in vivo beta cell quantification is impossible due to the small size of beta cells and islets of Langerhans, and the scattered distribution of islets throughout the entire pancreas. It was shown that the pancreatic beta cell mass in rats correlates with the amount of radioactivity in the pancreas, which means that, at least from a biological perspective, the uptake can be used as a measure for the beta cell mass.

Type 1 diabetes (T1D) patients generally have a lower beta cell mass than healthy humans, therefore the uptake in T1D patients was compared with the uptake in healthy volunteers. Quantification of the images showed indeed lower uptake in the T1D patients and variations in uptake of ${ }^{111}$ In-exendin within both groups that are in line with variations in beta cell mass in healthy volunteers and T1D patients. This indicates that radiolabeled exendin can indeed be used as a biomarker for the beta cell mass in human. SPECT quantification showed to be independent of the imaging time point, since the ${ }^{111}$ In remained in the pancreas for up to one week and in T1D patients. We conclude that also from a practical point of view (uncomplicated labeling, timing of the imaging not critical, despite the scan duration of 45 minutes subjects passed the study without difficulties), use of radiolabeled exendin for beta cell quantification is feasible.

The final validation of the use of radiolabeled exendin for beta cell quantification can only be given by studying ex vivo patient material and correlate this with beta cell mass (in a set-up similar as we performed on pre-clinical matera in chapter 4). Currently such a study, in which patients undergoing pancreatectomy will be administered with radiolabeled exendin and uptake in the pancreas will be evaluated quantitatively, is in preparation. The amount of activity in a part of the (ex vivo) pancreas will be measured in a gamma counter and compared to the beta cell mass in this section.

Quantification challenges for the clinical study of chapter 4 and 5 are discussed in chapter 6. In this work an anthropomorphic phantom including two kidneys and the pancreas, inserted in an existing body casing, was designed and used for 
simulation of the exendin imaging with SPECT. It demonstrated that the use of a 3D-printed phantom, mimicking the patient's anatomy, is helpful in clarifying whether a certain image characteristic is an artifact or not. In the phantom it appeared that the kidney cortex had a higher activity, however in real each kidney was one compartment with one activity concentration. Therefore a higher intensity in the cortex in the patient does not need to originate from a higher activity, as it can result from the reconstruction. Also inhomogeneity in the pancreas was observed in most reconstructions of the phantom, while the pancreas was filled homogeneously with one activity concentration. The phantom enabled optimization of the SPECT acquisition and reconstruction protocol for beta cell quantification with ${ }^{111}$ In-exendin.

This work demonstrated that absolute quantification in the pancreas with relatively low activity is challenging. More phantom scans would be required to investigate whether a calibration curve can be used for absolute quantification. For the time being, we chose to use the intensity in the pancreas for representation of the results of the clinical study, and to compare the intensity between subjects.

From the phantom studies we know that a higher activity in the pancreas indeed leads to a higher intensity, but the precise calibration factor seems to depend on the amount of activity in the pancreas. Therefore for future absolute quantification of the uptake in the pancreas more phantom scans need to be made to investigate if a calibration curve can be used for absolute quantification.

This work demonstrated how the use of a phantom, in this case a 3D-printed phantom, can contribute to the quantification of SPECT data and understanding of data

In chapter 7, different quantification and dosimetry techniques, not only based on gamma-camera images, but also on autoradiography, were combined to answer one single question: what is the radiation dose to the islets of Langerhans due to administration of radiolabeled exendin? Since exendin-based beta cell quantification relies on the uptake of the radiotracer in the beta cells, this method also leads to a certain radiation dose to the islets. To estimate the influence of the radiation dose, on the function of the beta cells, first a method for calculation of the islet radiation dose had to be developed.

In this chapter it was shown that useful quantification requires more than calculations: to answer this specific quantitative question, knowledge from different fields (physiology and dosimetry), and preclinical and clinical material, imaging data, histological data and autoradiography data was needed. This was combined in one model, developed for calculation of the islet dose due to administration of radiolabeled exendin.
Finally, the islet dosimetry model developed, was used to estimate variations in the dose induced by biological variations (having a higher amount of activity in the kidneys or more beta cells) and using a ${ }^{68} \mathrm{Ga}$ instead of ${ }^{111} \mathrm{In}$. In all simulated situations beta cell doses were much lower than the assumed toxicity levels.

We demonstrated a few examples of estimates that can be done with this model. The islet dosimetry model was developed in such a way that other researchers can add variations to the model and for example change islet sizes or add more radionuclides. By in the future replacing the preclinical input data by clinical data, the value of the model could be further improved.

The current model can be used to investigate the effect of differences in the rotocol (other radionuclide, amount of administered activity) and biologica variations (different islet sizes, percentages of beta cells in the islets, amount of activity in the exocrine pancreas, etcetera). In the future it can also be adapted to calculate the radiation dose to the beta cells for other radionuclides or even be modified for other tracers such as for example $\left[{ }^{11} \mathrm{C}\right] 5$-Hydroxy-Tryptophan $\left(\left[{ }^{11} \mathrm{C}\right] 5-\mathrm{HTP}\right)$

The studies described here, demonstrate that although there are challenges in the quantitative use of SPECT images, the applications of the technique are so valuable for answering different clinical questions, that it is worthwhile to tackle them. It showed to be worthwhile to think about methods to cope with uncertainties, and if absolute quantification is a bridge too far, to consider relative quantification, which can still largely contribute to answering clinical questions. 
Summary

Samenvatting

Dankwoord

About the author 


\section{Summary}

Current developments enabling quantitative use of single photon emission computed tomography (SPECT) images are valuable, because they facilitate broade use of quantification in nuclear medicine by enabling the use of single gammaemitting radionuclides in addition to positron-emitters.

In some cases, using a gamma-emitting radionuclide instead of a positronemitter is advantageous or even necessary; the selection of the radionuclide, and therefore the imaging modality (positron emission computed tomography (PET) or SPECT), is based on both technical, practical and safety concerns.

Therefore it is worthwhile to develop methods coping with uncertainties in SPECT, since they can efficiently contribute to answering clinical questions in regard to dosimetry and quantification of radiotracer distribution. In this thesis, challenges of SPECT for quantification and dosimetry are described and it is shown how, despite these challenges, quantitative use of SPECT is valuable for answering clinically relevant questions.

In chapter 1 it is shown why, although both positron emission tomography (PET) and SPECT can be used for quantification, quantification of SPECT images is more challenging. SPECT imaging requires the use of a collimator and the detectors rotate around the patient (instead of using a full ring detector system as in PET), therefore detection efficiency is lower. Also adequate correction for attenuation is more complicated. The energy windows selected for acquisition and the collimato used depend on the radionuclide. Fortunately nowadays advanced reconstruction methods can be applied for improving the quality of the quantification by providing corrections for scatter, attenuation and collimator modeling.

Besides the acquisition and reconstruction protocol, patient specific factors (e.g. movement) and practical issues (e.g. using software, which is not compatible with scaling factors in DICOM files of other vendors) can influence quantification. Also, for reliable interpretation of the quantification results, biological influences should be understood: what kind of tissue is actually determines the uptake and how could the uptake be influenced? This defines how the uptake we are quantifying should be interpreted.

When taking these factors into consideration, absolute or relative quantification of SPECT images can be performed, and radiation doses can be calculated.

In the chapters 2-7 quantification and dosimetry are applied for different purposes In chapter 2 accuracy of individualized dosing, based on pre-therapy administration with ${ }^{111}$ In-peptide, as surrogate for the pre-targeted radioimmunotherapy (PRIT) with ${ }^{177}$ Lu-peptide, was investigated. The relatively uncomplicated blood-based 
dosimetry and planar image-based dosimetry methods were applied to calculate the amount of ${ }^{177} \mathrm{Lu}$ that can be safely administered for PRIT. The study showed that with this approach indeed high amounts of activity can safely be administered, with only two out of twenty patients developing grade 3-4 hematological (platelet) toxicity.

In chapter 3, three-dimensional radiobiologic dosimetry (3D-RD) was applied for retrospective dosimetry for further investigation of the bone marrow toxicity that some patients developed in the PRIT study. A method was developed for using the 3D-RD software to calculate the bone marrow dose based on the activity in lumba vertebrae. Results were compared with blood-based and planar image-based bon marrow dosimetry results. 3D-RD based red bone marrow (RBM) dosimetry showed to be a more sensitive predictor for selection of patients showing any grade of RBM toxicity than planar image-based or blood-based dosimetry.

In addition to the RBM dosimetry, tumor dosimetry was performed, which was not possible based on the planar images. ${ }^{177} \mathrm{Lu}$ tumor-to-RBM dose ratios wer calculated and compared with simulated ${ }^{90} \mathrm{Y}$ dose ratios, to estimate the benefit of using ${ }^{90} \mathrm{Y}$ instead of ${ }^{177} \mathrm{Lu}$. Despite the increase in tumor-to-RBM dose ratio with ${ }^{90} \mathrm{Y}$, tumor doses remained disappointingly low. Although this could be interpreted as negative result, it was very useful in planning of future research in this field: similar clinical study with ${ }^{90} \mathrm{Y}$ was disregarded.

This example, in which the use of SPECT enabled more advanced RBM dosimetry and calculation of tumor doses, demonstrates the benefit of using SPECT images for dosimetry purposes.

In chapter 4 and 5 , SPECT is used for quantification of the amount of pancreatic beta cells. Beta cells are the insulin producing cells in the pancreas, and play a key role in diabetes. While beta cell function can be measured easily (e.g. by monitoring blood glucose), addition of a method for non-invasive in vivo quantification of the beta cell mass (BCM) is expected to significantly contribute to the knowledge about the pathophysiology and treatment of diabetes. The very small size and the scattered distribution of the beta cells and islets of Langerhans throughout the entire pancreas complicate imaging. The islets can only indirectly be visualized in vivo by measuring the uptake of a highly beta cell specific imaging tracer. Therefore radiolabeled exendin, which specifically targets the glucacon-like-1 (GLP-1) receptor, expressed on beta cells, was developed. Exendin labeled with ${ }^{111}$ In can be visualized in vivo with SPECT. In chapter 4 it was shown that pancreatic BCM in rats correlates with the amount of radioactivity in the pancreas, which means that, at least from a biological perspective, the uptake can be used as a measure for the beta cell mass. In humans, a proof of principle study was conducting demonstrating that the uptake of the radiotracer in the human pancreas can be quantified and differences between healthy individuals and patients with diabetes can be visualized.

Type 1 diabetes (T1D) patients generally have a lower beta cell mass than healthy humans, therefore in the first complete clinical study about the use of radiolabeled exendin for in vivo beta cell quantification, the uptake of ${ }^{111}$ In-exendin in pancreata of ten T1D patients and ten healthy volunteers was quantified with SPECT. In chapter 5 , quantification of these images showed indeed lower uptake in the T1D patients and variations in uptake of ${ }^{111}$ In-exendin within both groups that are in line with variations in beta cell mass in healthy volunteers and T1D patients. This indicates that radiolabeled exendin can indeed be used as a biomarker for the beta cell mass in human.

Since exendin has a relatively high uptake in the kidneys compared to the pancreas, quantification of the pancreatic uptake is even more complicated than SPECT quantification in general. Therefore the phantom work described in chapter 6, was performed. A 3D-printed anthropomorphic phantom with two kidneys and a pancreas was developed, to mimic the patient situation. This enabled thorough investigation of the effect of different settings in reconstruction software and ptimization of the image reconstruction protocol. It demonstrated that the use of a 3D-printed phantom is helpful in clarifying whether a certain image characteristic is an artifact or not. Also, the phantom enabled optimization of the SPECT acquisition and reconstruction protocol for beta cell quantification with ${ }^{111}$ In-exendin.

This work demonstrated how the use of a phantom, in this case a 3D-printed phantom, can contribute to the quantification of SPECT data and understanding of data.

In chapter 7, different quantification and dosimetry techniques, not only based on gamma-camera images, but also on autoradiography, were used for calculation of the radiation dose to the islets of Langerhans after administration of radiolabeled exendin. In this chapter it was shown that useful quantification requires more than only a few calculations. In order to exactly determine the dose to the islets, knowledge from different fields (physiology and dosimetry), and preclinical and clinical data (imaging data, histological data and autoradiography data) were needed. This complex set of information was integrated in one dosimetric model, developed for calculation of the islet dose due to administration of radiolabeled exendin

Finally, the developed islet dosimetry model was used to estimate the influences on the radiation dose induced by biological variations (being a male or 
a female, having a higher amount of activity in the kidneys or more beta cells) and using a ${ }^{68} \mathrm{Ga}$ instead of ${ }^{111} \mathrm{In}$.

This work demonstrates that although we face numerous challenges in the quantitative use of SPECT images, the applications of the technique are so valuable for answering different clinical questions, that it is worthwhile to tackle them. 


\section{Samenvatting}

In dit proefschrift wordt beschreven wat de uitdagingen zijn bij kwantitatieve analyse van 'single photon emission computed tomography' (SPECT) beelden en het gebruik van deze beelden voor het berekenen van de stralingsdosis (dosimetrie). Ondanks deze uitdagingen is het aantrekkelijk om SPECT te gebruiken voo bepaalde kwantitatieve toepassingen. Daarom wordt in dit proefschrift beschreven hoe omgegaan kan worden met deze uitdagingen en hoe kwantitatieve SPECT en dosimetrie kunnen bijdragen aan klinisch relevante vraagstukken.

Hoofstuk 1 gaat in op de uitdagingen die er zijn bij het kwantitatief gebruik van SPECT, en mogelijkheden van kwantitatieve SPECT en dosimetrie.

In de nucleaire geneeskunde worden twee beeldvormingsmethoden veelvuldig gebruikt: SPECT en 'positron emission tomography' (PET). Er zijn grote verschillen tussen beide technieken, met name in hoe het beeld wordt verkregen.

Bij PET komt het uitgestraalde positron samen met een elektron, hierbij komen twee fotonen vrij die in tegengestelde richting worden uitgezonden. Dit heet annihilatie. De twee fotonen worden gelijktijdig gedetecteerd door de PET camera.

In SPECT worden losse fotonen (gammastraling) die zijn uitgezonden door de radioactieve stof gedetecteerd. Om van deze gedetecteerde fotonen een beeld te kunnen maken is voor de detector een raster (collimator) geplaatst dat enkel loodrecht invallende fotonen doorlaat. Hoewel gebruik noodzakelijk is, is een nadeel hiervan dat de detectie efficiëntie hierdoor wordt verlaagd.

Tevens wordt het gemeten signaal beïnvloed door het weefsel dat de fotonen moeten doorkruisen alvorens het de detector bereikt. Een deel van de fotonen wordt geabsorbeerd, en een deel van de fotonen wordt afgebogen (gescatterd). Hiervoor moet worden gecorrigeerd om de SPECT beelden kwantitatief te kunnen gebruiken

Recentelijke ontwikkelingen in reconstructie methoden, die worden gebruikt om van het gedetecteerde signaal een driedimensionaal (3D) beeld te maken, maken het mogelijk om voor de absorptie, de scattering en het gebruik van de collimator te corrigeren. Hierdoor wordt de kwaliteit van de SPECT beelden vee beter, waardoor ze betrouwbaarder kunnen worden gebruikt voor berekeningen.

Het naast PET ook SPECT kunnen gebruiken voor kwantificatie en dosimetrie is aantrekkelijk, omdat daarmee andere radioactieve stoffen gebruikt kunnen worden. Soms heeft het de voorkeur, of is het zelfs noodzakelijk om een gammastraler te gebruiken, vanwege praktische, technische of veiligheidsredenen.

Ondanks de uitdagingen die het gebruik van SPECT voor kwantificatie of dosisberekeningen met zich mee brengt, biedt het gebruik van SPECT vele mogelijkheden. Daarom is het waardevol om deze uitdagingen aan te gaan. 
In hoofdstuk 2 tot en met 7 worden dosimetrie en kwantificatie voor verschillende doeleinden gebruikt.

In hoofstuk 2 wordt dosimetrie gebruikt om te bepalen wat de veilige hoeveelheid radioactiviteit is die kan worden toegediend aan patiënten die behandeld worden met ${ }^{177} \mathrm{Lu}$. Relatief eenvoudige dosimetrie methoden, gebaseerd op tweedimensionale beelden en meting van bloedmonsters, worden gebruikt on te bepalen hoeveel activiteit veilig kan worden toegediend voor de behandeling. Met deze methoden wordt berekend welke stralingsdosis op het beenmerg word verwacht. Hoge beenmerg doses zijn onwenselijk, omdat het beenmerg een erg stralingsgevoelig orgaan is, waarin bloedcellen moeten worden aangemaakt. Di onderzoek laat zien dat door gebruik van deze methoden inderdaad veilig grote hoeveelheden radioactiviteit kunnen worden toegediend. Slechts twee van de twintig patiënten hebben na behandeling 'graad 3-4' verlaagde hoeveelheden bloedplaatjes, door stralingsschade aan het beenmerg.

In hoofdstuk 3 wordt een andere methode voor de beenmerg dosimetrie gebruik voor dezelfde groep patiënten. In dit hoofstuk wordt de dosis met terugwerkende kracht berekend, op basis van SPECT beelden die gemaakt zijn gedurende de behandeling. Om verder inzicht te krijgen in de relatie tussen de beenmerg dosis en de schade aan het beenmerg (verlaagde waarden van de bloedplaatjes) is een nieuwe methode ontwikkeld met de zogenaamde three-dimensional radiobiologic dosimetry (3D-RD) software. In SPECT beelden worden verschillende lumbale wervels ingetekend, waarin beenmerg zit. Op basis hiervan worden stralingsdoses op het beenmerg berekend. Het blijkt dat deze methode gevoeliger is voor het voorspellen van enige mate van beenmerg schade dan de eenvoudigere methoden.

Een ander voordeel van het gebruik van deze methode is dat het ook tumo dosimetrie mogelijk maakt. Hiermee kan de ratio worden berekend tussen de tumor dosis en de beenmerg dosis. Een hogere ratio is gunstig voor het te verwachten therapeutisch effect van de behandeling ten opzichte van de te verwachten schade aan het beenmerg. Zo is het mogelijk om een inschatting te maken of gebruik van ${ }^{90} \mathrm{Y}$ in plaats van ${ }^{177} \mathrm{Lu}$ tot een beter behandelingsresultaat zou leiden. Hoewel deze ratio inderdaad gunstiger uitpakt voor ${ }^{90} \mathrm{Y}$, zou de tumor dosis in alle patiënten laag blijven. Dit is een nuttig resultaat, omdat hiermee een volgend onderzoek met patiënten, met ${ }^{90} \mathrm{Y}$ voorkomen kon worden.

Dit voorbeeld laat zien waarom SPECT van toegevoegde waarde is voo beenmerg dosimetrie en wat de waarde is van het kunnen berekenen van tumor dosis op basis van SPECT.

In de hoofdstukken 4 en 5 wordt SPECT gebruikt voor het kwantificeren van de bèta cellen in de alvleesklier. De bèta cellen zijn de insuline producerende cellen, die een belangrijke rol spelen in suikerziekte. Wanneer het mogelijk is om de hoeveelheid van deze cellen te meten zonder hierbij een deel van de alvleesklier te verwijderen uit de patiënt, kan de rol van deze cellen in de ontwikkeling en behandeling van suikerziekte verder worden onderzocht. Omdat deze cellen en de eilandjes van Langerhans waar ze in zitten zo klein zijn, is het lastig om deze te meten. Daarom is radioactief gelabeld exendin ontwikkeld. Exendin wordt via de zogenaamde 'GLP-1' receptor, die voornamelijk op de bèta cellen zit, opgenomen in de bèta cellen. Door het radioactieve label is het mogelijk om de bèta cellen indirect, met een SPECT camera, in beeld te brengen.

In hoofdstuk 4 wordt de hoeveelheid bèta cellen in de alvleesklier van ratten ergeleken met de hoeveelheid radioactiviteit in de alvleesklier na toediening van radioactief exendin. Hieruit blijkt een goede relatie tussen de hoeveelheid radioac tiviteit en de hoeveelheid bèta cellen. Dit is een aanwijzing dat, na toediening van het radioactieve exendin, de gemeten hoeveelheid radioactiviteit in de alvleesklie gebruikt kan worden als maat voor hoeveelheid bèta cellen

Patiënten met type 1 suikerziekte hebben over het algemeen minder bèta cellen dan gezonde mensen. Daarom worden in hoofdstuk $\mathbf{5}$, in de eerste klinische studie waarin radioactief exendin wordt gebruikt voor kwantificatie van de bèta cellen, patiënten met type 1 suikerziekte en gezonde vrijwilligers vergeleken. De tien deelnemers in beide groepen kregen radioactief exendin, waarna verschillende SPECT scans werden gemaakt. Hieruit bleek dat de gemeten gemiddelde hoeveelheid radioactiviteit in de alvleesklier van de gezonde vrijwilligers inderdaad hoger was dan die in patiënten met type 1 suikerziekte. De variatie binnen beide groepen komt overeen met de variatie in de hoeveelheid bèta cellen die bekend zijn uit literatuur. De resultaten van dit zogenaamde 'proof of principle' onderzoek in mensen bevestigen dat radioactief exendin mogelijk gebruikt kan worden voo kwantificatie van de hoeveelheid bèta cellen. Hierdoor kan kwantitatieve SPECT van radioactief exendin een belangrijke bijdrage leveren aan verder onderzoek naar de ontwikkeling en behandeling van suikerziekte.

Omdat vergeleken met de alvleesklier relatief veel exendin (en dus ook radioactiviteit) in de nieren zit, is kwantificatie van de opname in de alvleesklier nog lastige dan SPECT kwantificatie in het algemeen. Daarom wordt in hoofdstuk 6 een 3D-geprint fantoom van nieren en een alveesklier gebruikt om het SPECT scannen van de patiënten te kunnen simuleren. Hiermee kan het effect van verschillende instellingen voor het maken en reconstrueren van de beelden op de kwantificatie worden ingeschat, en konden protocollen worden geoptimaliseerd. Tevens kon hiermee duidelijkheid worden verkregen over wat werkelijk afwijkingen zijn en wat artefacten zijn in de beelden. 
Dit werk liet zien hoe het gebruik van een 3D-geprint fantoom kan bijdragen aan de kwantificatie van SPECT beelden en het begrijpen van de data.

In hoofdstuk 7 worden verschillende methoden voor kwantificatie en dosimetrie, niet alleen gebaseerd op beelden van een gamma-camera, maar ook op basis van autoradiografie, gebruikt voor het berekenen van de stralingsdosis op de eilandjes van Langerhans. Dit werk laat zien dat er meer nodig is voor nuttige kwantificatie dan enkel berekeningen: om deze specifieke kwantitatieve vraag te kunnen beantwoorden was het nodig om kennis uit verschillende vakgebieden (fysiologi en dosimetrie), preklinische en klinische data (beelden, histologisch materiaal en autoradiografie) te combineren. Dit werd samengebracht in een model voor de berekening van de stralingsdosis op de eilandjes door gebruik van radioactie exendin. Met dit model werden stralingsdosis voor verschillende biologische variaties (meer of minder activiteit in de nieren, meer of minder bèta cellen in de alvleesklier, man of vrouw) en voor ${ }^{68} \mathrm{Ga}$ en ${ }^{111}$ In berekend. De stralingsdosis op de eilandjes van Langerhans bleef ver onder de limiet waarboven we stralingsschade kunnen verwachten. Dit laat zien dat beeldvorming, waaronder SPECT, en dosimetrie ook voor het maken van dergelijke complexe berekeningen en inschattingen uiterst waardevol is

Dit alles leert ons dat ondanks de uitdagingen, er ook uitgebreide kansen zijn voor het kwantitatief gebruik van SPECT beelden en dosimetrie. De mogelijke toepassingen, waarvan dit proefschrift enkele voorbeelden beschrijft, zijn zo waardevol dat het het waard is om de uitdaging aan te gaan en kwantitatieve SPECT en dosimetrie te gebruiken in de praktijk. 


\section{Dankwoord}

Ik was van plan om een kort dankwoord te schrijven. Dat is niet gelukt..

Vier jaar geleden was het absoluut niet mijn ambitie om onderzoek te gaan doen, laat staan te promoveren. Toch ben ik nu wel dank verschuldigd aan de mensen doo wie ik ben gebleven op mijn afstudeerplek, want het was leuk en leerzaam! Eric, ik bleef, want je luisterde naar mijn wensen en dacht mee over opties om niet alleen onderzoek te doen, Martin, jij bood me een klinisch onderzoek aan met veel praktisch werk (leuk!), en misschien nog wel het aller belangrijkst: dank aan diegenen die het fellowship mogelijk maakten, want het fellowship gaf de doorslag om te blijven.

De afgelopen jaren waren leerzaam, interessant, gezellig, leuk en soms een beetje hectisch (wat wil je als je ook privé zo veel mooi life-events in een paar jaar stopt...). Gelukkig kan ook zo'n tijd behoorlijk soepel verlopen, als alles om je heen ongeveer klopt. Daaraan hebben heel veel mensen een steentje bijgedragen.

Ten eerste Eric: de afgelopen jaren was je mijn co-promotor en leidinggevende (zowel voor mijn promotie als het fellowship). In beide trajecten zochten we naar de beste invulling voor mij. Je gaf me de ruimte én was oprecht betrokken, zowel inhoudelijk, als praktisch: meer dan ikzelf jij had begrip voor de situatie als ik vanwege een zwangerschap of als Hidde ziek was even minder kon werken dan ik eigenlijk wilde. Dat had ik denk ik nodig: bedankt!

Martin, je duizend-en-een ideeen, je overtuigingskracht wanneer ik twijfelde aan mijn eigen kunnen, en de mogelijkheden die je me als promotor bood, waren cruciaal voor deze promotie. Na eerst het solitaire dosimetrie-werk, gaf je me met het Exendin-deel van mijn promotie de mogelijkheid aan te sluiten bij jouw Beta-image groep: bedankt hiervoor

Kees, op afstand, maar zeker wel betrokken! Eens in de paar maanden overlegden we, maar dan hielp juist je frisse blik ons scherp te blijven. Het 3D-geprinte fantoom was er niet gekomen, en vele kleine foutjes (komma's, waar punten horen) waren lijven bestaan. Bedankt dat je mijn promotor wilde zijn!

Mijn kamergenootjes Charlotte, Inge, Maarten, Marlène, Peter, Rafke, Sandra, Stefanie, Stijn, Tessa, Waël en Willem, de rest van de beta image groep, Samantha en andere (oud-)collega's van de nucleaire researchgroep: bedankt voor alle gezelligheid, support, hardloop-afspraken, fiets-vergader-verzoeken, bier-etentjes en het ongegeneerd heel veel kunnen snoepen (banaantjes!!). Ik kan wel een pagina vol schrijven ove hoe leuk en fijn het was, maar jullie weten allemaal goed wat ik bedoel. Ik hoop dat al het goeds binnen de afdeling altijd zo goed blijft 
Rafke, aan het eind van jouw promotie en het begin van mijn onderzoek gingen we samen naar Baltimore. Daar was zelfs oversteken van het ene naar het andere gebouw zonder bewaker was met jou een bijzonder avontuur. Ik kijk met plezier terug op die tijd en onze samenwerking, en ik wens je in het werk en privé veel geluk!

Professor Sgouros (George) and Rob, many thanks for the opportunity you gave us to work with 3D-RD. It took longer than expected, but I'm glad that now we published our nice paper! Thanks for your help!

ledereen die mee heeft geholpen aan de Exendin-studie: jullie weten als geen ander dat een klinische studie teamwork is. Volgens mij heeft de hele afdeling we iets gedaan, al was het maar een kamertje bij de PET vrij houden voor de screening of glucosedrankjes bestellen.

Martin, jij zette deze studie op, en gaf mij de kans het stokje in de uitvoering over te nemen nadat het protocol was goedgekeurd. Als investigator bleef je nauw betrokken. Cees, Bastiaan en andere artsen van de interne geneeskunde: bedank voor jullie inhoudelijke ondersteuning en jullie hulp bij de recrutering van patiënten!

Jacqueline, Marie-Louise en Manon: we werden overspoeld met telefoontjes van te oude te dikke mensen die zich graag als gezonde vrijwilliger inschreven. Dank voor jullie hulp bij het afwijzen en voor al jullie andere hulp (ook voor de 1001 lastige afspraken die jullie voor mij inplanden!!!!).

Judith, Lisette en Eddy: ik kwam altijd met ambitieuze wensen, namelijk zo snel mogelijk, heel vaak achter elkaar de drukste camera bezet houden. Julli zeiden nooit nee, en zochten altijd mee naar de beste optie: bedankt voor jullie flexibiliteit en hulp! MNW'ers: Eddy, Jur, Marga, Marjo, Marie-Claire, Detje, Martin E, Merijn, Michel en Diana bedankt voor jullie behulpzaamheid, zorgvuldigheid bij het maken van de scans en het lief zijn voor de vrijwilligers die zo lang moesten liggen!

Maichel, Miranda en Natascha, bedankt voor de goede samenwerking. Bijn alle bereidingen gingen in één keer goed en zo konden we alles vooruit plannen zonder ons zorgen te maken!

AIOS die hielpen bij de screening en stafleden die assisteerden bij de toediening: ook bedankt voor jullie hulp.

TG-studentes die hielpen bij de controles toen ik, zwanger zijnde, beter niet uren bij de radioactieve proefpersonen kon doorbrengen: dank jullie wel!

Marti, samen hebben we alle scans nogmaals geanalyseerd op de IRW: ik wens je een mooie onderzoekstijd toe en jou, Mijke en Laura ook veel leuke patiënten en mooie Exendin-scans!
Maarten en Otto: bedankt voor het meedenken wanneer er iets anders ging dan we hadden verwacht en bedankt voor jullie support. Fijn om te weten dat jullie altijd voor de onderzoekers klaar staan!

Marcel, vanaf het begin was je betrokken bij de studie. We deden de eerste screeningen samen (ons beiden afvragend of we nou alles goed hadden gedaan), je nam later zelfs al het werk van me over tijdens mijn verlof en je was altijd beschikbaar om mee te denken over praktische zaken. Heel erg veel dank dat je altijd mijn backup was! Ik hoop dat we in het fellowship een net zo'n mooie en productieve tijd samen tegemoet gaan.

Maaike, Laura D en Laura P, jullie deden alle drie een M2 TG stage bij ons op de afdeling en hebben tijdens deze stages het fantoom ontworpen en verschillend experimenten gedaan. Het was voor mij leerzaam om jullie hierbij te begeleiden, maar ik ben heel blij dat jullie wel studenten waren die heel goed en zorgvuldi werk leveren: daardoor was het mogelijk om het 'fantoom-paper' te schrijven. Bedankt voor jullie mooie bijdrage!

Inge, 'het dosimetrie-model' is een beetje onze gezamenlijke trots. Toen we hiermee echt aan de slag gingen, werkten we binnen een paar maanden van een idee, naa resultaten, naar een paper. Ik vond het super fijn dit werk met jou te kunnen doen. We vulden elkaar perfect aan, hielden elkaar scherp, vonden het beiden leuk weer wat meer met MATLAB en formules te stoeien, en zelfs schrijven konden we goed samen. Bijna jammer dat het nu klaar is hè?

Wim Oyen, officieel was je alleen als co-auteur betrokken bij deze promotie. Ik vin het altijd knap hoe je perfect de vinger op zere plek kan leggen en zo goed en snel commentaar kan leveren op papers. In werkelijkheid was je invloed de afgelopen jaren groter, als een van de drijvende krachten achter het fellowship. Heel erg veel dank voor de mogelijkheden die je me bood, deuren die je opende en dat ik altijd mocht langskomen voor advies. Jammer dat je weg gaat..

Antoi, officieel was je misschien niet bij alles betrokken, maar in de praktijk was je dat juist wel. Jij kenmerkt je door de rust, het geduld, je praktische benadering en je grote mate van behulpzaamheid. Het is een fijn om jou in de buurt te hebben.

Malou en Marja, onder het mom van even lunchen of theedrinken hebben we de nodige problemen opgelost of voorkomen. Het is een luxe als vriendinnen ook op het werk zo dicht bij zijn! Op naar de volgende promotie! Of baby ;) 
Lieve familie, vrienden en buren: het was fijn dat jullie de afgelopen tijd zo behulpzaam waren. Waarschijnlijk hadden jullie het zelf niet eens altijd door, maar door bij ons te koken, even op Hidde te passen, mee te gaan hardlopen, wielrennen, geïnteresseerd te zijn, mee te leven en me bewust te houden dat er belangrijkere dingen zijn dan werk, hebben jullie een substantiële bijdrage geleverd aan dit boekje. Ik hoop dat we ons Bourgondische, sportieve en heerlijk burgerlijke bestaan nog lang met jullie mogen delen.

Heit en mem, ook al vind ik het soms lastig in begrijpelijke taal uit te leggen wat ik doe, jullie zijn oprecht geïnteresseerd, trots, en begrijpen als geen ander wanneer we even een steuntje in de rug nodig hebben. Het is heel fijn om te weten dat jullie achter ons staan en dat we altijd kunnen bellen. Soms is dat beetje praktische hulp (waarbij jullie het zelfs laten lijken alsof wij jullie een gunst verlenen...) nét dat wat we nodig hebben..

En natuurlijk bedank ik als laatste Yoël! Pop, zolang je het maar uit je hoofd laa om ooit zelf te gaan promoveren, zal ik jou altijd in het werk blijven steunen zoals jij mij steunt met mijn werk. Namelijk genoeg, maar niet té veel: werk is immers ook maar werk, en je moet af en toe ook de tijd nemen om lekker te koken, te sporten of samen niks te doen. Je hebt gelijk, door me soms een beetje af te remmen gaat het alleen maar beter. Ik ben er trots op dat we zo'n goed team zijn, en geniet erg van jou, Hidde en de wurm (-)

\section{Wietske}

April 2015 


\section{About the author}

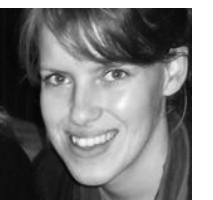

Wietske van der Weg was born on 27 March 1987 in Leeuwarden, the Netherlands. She grew up in the north of Friesland on her parents' potato farm. In 2005 she graduated from the Piter Jelles Gymnasium in Leeuwarden and moved to Enschede to study Technical Medicine at the University of Twente.

While attending University, she was also an active member of the student rowing club DRV Euros, where she was both a competitive rower and an enthusiastic cox. After obtaining her Bachelor's degree in 2008, she started the technical medicine Master's program, specializing in 'Robotics and Imaging'. In 2009 she joined a study-tour to Japan. As this study break was way too short, she hopped on a plane from Japan to New Zealand for the backpacking experience of a lifetime followed by a short detour visiting relatives in the US. Her Master's thesis wa entitled 'Use of prospective and retrospective, patient-specific, 3D tumor and red bone marrow dosimetry in nuclear medicine'. The majority of this work was performed at the at the Department of Radiology and Nuclear Medicine at the Radboud University Medical Center. She had the pleasure of working one month of her final internship at the John's Hopkins Medical Centre in Baltimore (MD, USA) under supervision of professor George Sgouros.

In 2012 she received her Master's degree and was offered the opportunity to not only continue her work on dosimetry in a PhD project, but she was also appointed the first 'Fellow Technical Medicine' in the Netherlands.

In 2013 she married Yoël Woliner and their son Hidde was born.

After finishing her PhD, she will continue pioneering as a fellow at the Department of Radiotherapy and the department of Radiology and Nuclear Medicine of the Radboud University Medical Center. 
